\title{
Polarimetric characterization of light and media
}

\section{Physical quantities involved in polarimetric phenomena}

\author{
J.J. Gil ${ }^{\mathrm{a}}$ \\ Universidad de Zaragoza, ICE c/ Pedro Cerbuna 12, 50009 Zaragoza, Spain
}

Received: 11 June 2007 / Received in final form: 16 July 2007 / Accepted: 26 July 2007 Published online: 3 November 2007 - C EDP Sciences

\begin{abstract}
An objective analysis is carried out of the matricial models representing the polarimetric properties of light and material media leading to the identification and definition of their corresponding physical quantities, using the concept of the coherency matrix. For light, cases of homogeneous and inhomogeneous wavefront are analyzed, and a model for 3D polarimetric purity is constructed. For linear passive material media, a general model is developed on the basis that any physically realizable linear transformation of Stokes vectors is equivalent to an ensemble average of passive, deterministic nondepolarizing transformations. Through this framework, the relevant physical quantities, including indices of polarimetric purity, are identified and decoupled. Some decompositions of the whole system into a set of well-defined components are considered, as well as techniques for isolating the unknown components by means of new procedures for subtracting coherency matrices. These results and methods constitute a powerful tool for analyzing and exploiting experimental and industrial polarimetry. Some particular application examples are indicated.
\end{abstract}

PACS. 42.25.Ja Polarization - 42.68.Mj Scattering, polarization

\section{Introduction}

A proper description of the polarization properties of electromagnetic waves relies on the concept of the coherency matrix. This mathematical formulation is applicable regardless the particular band of the electromagnetic spectrum considered. In this paper we will frequently refer to "light" but, except for particular cases which are identified from the context, this term can be substituted by "electromagnetic radiation". In fact there are many important industrial and research subjects involving polarization of electromagnetic radiation beyond the optical range such as microwaves, $\mathrm{X}$-rays and gamma-rays.

Given a fixed point in space, the electric field of an electromagnetic wave describes an ellipse (the polarization ellipse) in a plane perpendicular to the propagation direction. For the ideal case of monochromatic light, both polarization ellipse and propagation direction are fixed. In general, polychromatic light behaves as monochromatic for time intervals longer than the natural period $T_{0}$ of the wave and shorter than the coherence time $\tau$, so that the following different situations can be distinguished:

- Both the propagation direction and the polarization ellipse are stable; in consequence, the light beam is fully polarized. For most purposes, and from a polarimetric point of view, monochromatic totally polarized light can be considered equivalent to monochromatic light.

\footnotetext{
a e-mail: ppgil@unizar.es
}

- The propagation direction is fixed, whereas the polarization ellipse fluctuates; in consequence, the light beam is partially polarized. It is well-known that these states are polarimetrically equivalent to a statistical mixture of two pure states with orthogonal polarizations. We will show that mixed states are also equivalent to statistical mixtures of non-orthogonal pure states.

- Both the propagation direction and the polarization ellipse fluctuate. As we will see, an objective measure of the degree of directionality can be defined, which is always higher than (or equal to) the objective measure of the stability of the polarization ellipse. These states require a particular treatment, different to that the commonly used for light with a fixed direction of propagation.

In general, concerning the optical band of the electromagnetic spectrum, the time of measurement $T$ (i.e. the response time of the detector) is much higher than the coherence time. Typical values of the indicated time intervals are the following: $T_{0} \equiv 1 / \nu_{0} \cong 10^{-15} \mathrm{~s}\left(\nu_{0}\right.$ being the central frequency of the spectral profile of the wave), $10^{-9} \mathrm{~s} \leqslant \tau \leqslant 10^{-4} \mathrm{~s}$ and $T \cong 10^{-4} \mathrm{~s}[1,2]$. Thus, for most purposes the assumption of quasimonochromaticity is justified.

In a second-order optics approach, the Stokes parameters [3] provide a complete mathematical characterization of the different states of polarization. The physical 
meaning of these four real parameters has been studied in depth by several authors [4-7].

When a material medium is irradiated by an electromagnetic wave, molecular electric charges are set in oscillatory motion by the electric field of the wave, producing secondary radiation, so that the overall effects of the combined basic interactions (scattering in its essential sense) [8] results in refracted, reflected, diffracted or scattered light. The effects of linear passive media can be represented by linear transformations of the electric field variables. By "passive" we refer to the property of not amplifying the light intensity.

Depending on: (1) the nature and particular conditions of the linear interaction; (2) the spectral profile of the incident light beam; (3) the chromatic properties of the material sample, and (4) the specific polarizing properties of the sample, the waves emerging from different homogeneous parts of the material target can have different degrees of mutual coherence. Coherent interactions can be represented through the Jones formalism [9], whereas for the general case of incoherent interactions of polychromatic light, the Stokes-Mueller model is required.

Concerning the transformations of the state of polarization due to the interaction of the wave with linear material media, Jones introduced a simple method applicable for deterministic interactions [9]. In this model, the polarization states are represented by two-component complex "Jones vectors" and the medium is characterized by a $2 \times 2$ complex "Jones matrix".

The classic contributions of authors such as Soleillet [10], Perrin [11], Mueller [12] and Parke III [13,14], have led to a more general model, in which the action of media is represented through linear transformations of the Stokes parameters. Although the $4 \times 4$ real matrices characterizing these transformations are usually called "Mueller matrices" it should be noted that it was Soleillet who first introduced this concept [10]. The lecture notes where Mueller used these matrices remained unpublished except for a brief note [12]. The connection between the Jones and the Soleillet-Mueller models was studied by Parke III $[13,14]$. An interesting compilation of several seminal works on polarization optics can be found in reference [15].

Some works have dealt with the case of deterministic nondepolarizing media (polarimetrically pure media), leading to clear physical interpretations of the seven independent parameters that appear in this case $[16,17]$. In the general case, a complete polarimetric characterization of the medium requires considering up to sixteen independent parameters.

Given the essential nature of polarization phenomena, polarimetry is a general-purpose technique which can be applied in a great variety of industrial, medical and scientific environments. Thus, different interaction conditions can be arranged in order to obtain information about material samples from polarimetric measurements. These techniques have been widely used for many years. One particular technique, called "ellipsometry" $[18,19]$, is based on determining some properties of material samples from the changes produced on totally polarized light. The interaction transforms the "input polarization ellipse", characterized by a complex number that contains information about the corresponding azimuth and ellipticity, into a reflected "output polarization ellipse". The ratio between these numbers results in two ellipsometric real parameters. Ellipsometric techniques provide very fast in-situ and nondestructive control of several industrial processes. Nevertheless, it should be noted that the term "ellipsometry" is also frequently used as a synonym for "polarimetry" and, hence, refers to general techniques for measuring polarimetric properties.

In general, interactions not only produce changes in the ellipsometric parameters, but also cause selective changes in the transmitted intensity as well as depolarizing effects, so that, in these cases, a complete polarimetry is required. For the measurement of all the sixteen elements of a Mueller matrix, the material sample is placed between a generator and an analyzer of polarization states. The generator must be arranged into at least four independent configurations for each one of which the analyzer must in turn be disposed into at least four independent configurations. Usually, the generator includes a total polarizer followed by a retarder placed in front of the light source, whereas the analyzer includes a retarder followed by a total polarizer before the detector. By means of rotating wave-plates, Pockels cells or other kinds of controllable retarders, these polarimeters can be automatically operated [20-28].

The analyzer described, used alone, can be used as a Stokes polarimeter, enabling the Stokes parameters of the incoming light to be measured [29]. Other interesting devices are based on the use of polarization gratings [30,31].

Different kinds of polarimeters are used in several different environments such as remote sensing [32]; scattering applications [8,33,34]; ITER \& Tokamak [35]; Astronomy [36], etc.

For some applications, spectral polarimetry and interferometry are combined into a whole device [35-40].

Polarimetric techniques are powerful tools for the study and analysis of material samples because, given the specific interaction conditions (reflection, refraction, scattering...) and given the spectral and spatial properties of the light probe, up to sixteen independent parameters can be measured. These measurable quantities are the elements of the Mueller matrix, which characterize the polarimetric properties of the sample with respect to the interaction conditions mentioned. Nevertheless, important limitations of these techniques arise from the absence of a complete understanding of the information contained in the Mueller matrix. The physical information is structured in a complicated manner and, thus, the obtainment of a set of sixteen quantities with clear physical meaning is not straightforward. In fact, as we will see, some new parameters must be introduced in order to reach the indicated objective.

Consequently, the mathematical characterization of Mueller matrices is a key question in the exploitation of powerful polarimetric techniques. In general, the 
values of the elements of Mueller matrices are restricted by several quadratic and bilinear constraining inequalities, which have been the main subject of several papers.

In this review, we consider the mathematical description of the polarimetric properties of light and media through a unified model based on the concept of coherency matrices. The measurable quantities arise as the coefficients of the expansion of the coherency matrix in a set of Hermitian trace-orthogonal matrices constituted by the generators of the $\mathrm{SU}(n)$ group plus the $n \times n$ identity matrix. The physical parameters with direct physical meaning are defined from the corresponding measurable quantities.

The different sections of this paper include relevant results quoted in the literature, as well as some new results, which are treated on the basis of the unified framework indicated [41]. Thus, for example, in the light of the respective "indices of purity", the polarimetric purity of $3 \mathrm{D}$ states of polarized light is analyzed, as well as the polarimetric purity of media. Moreover, the retarding, polarizing and depolarizing properties of material media are characterized through a set of sixteen independent parameters with direct physical meaning. We will find that ten of these parameters are physically-invariant quantities.

The following sections consider successively the polarization states of plane waves, the 3D states of polarization and the polarimetric properties of material media. In general, when possible, the habitual notations and names have been preserved. In some cases, particular notations have been introduced for the sake of clarity.

\section{Polarized light}

Given a point in space, the state of polarization of a light beam that propagates in a fixed direction $z$ is given by the temporal evolution of the electric field of the electromagnetic wave, which lies in a plane perpendicular to the propagation direction. Let $\mathbf{E}(z, t)$ be the electric field at a point $z$, at time $t$, of a monochromatic plane wave propagating in an isotropic medium, and let $\left(\mathbf{e}_{1}, \mathbf{e}_{2}, \mathbf{e}_{3}\right)$ be a reference basis of orthonormal vectors along the respective axes $X Y Z$. The components of the electric field are

$$
\begin{aligned}
& E_{x}(z, t)=A_{x} \cos \left(k z-\omega t+\beta_{x}\right) \\
& E_{y}(z, t)=A_{y} \cos \left(k z-\omega t+\beta_{y}\right)
\end{aligned}
$$

where $k=2 \pi / \lambda_{0}\left(\lambda_{0}\right.$ is the wavelength for the vacuum); $\omega$ is the angular frequency, $\omega=2 \pi \nu$ ( $\nu$ is the natural frequency); $\beta_{x}, \beta_{y}$ are the respective phases, and $A_{x}, A_{y}$ are the respective amplitudes.

In the case of quasimonochromatic light, i.e. when the bandwidth $\Delta \nu$ is very narrow with respect to the central frequency $\bar{\nu}$ of the spectral profile, the components of the electric field can be expressed as

$$
\begin{aligned}
& E_{x}(z, t)=A_{x}(t) \cos \left(\bar{k} z-\bar{\omega} t+\beta_{x}(t)\right) \\
& E_{y}(z, t)=A_{y}(t) \cos \left(\bar{k} z-\bar{\omega} t+\beta_{y}(t)\right)
\end{aligned}
$$

where $\bar{k}, \bar{\omega}$ are the respective mean values of $k, \omega$.
As usual in polarization optics, under this assumption of quasimonochromaticity, it is very advantageous to use the analytic signal representation, where the components are described through their respective complex variables [6].

Let us consider the analytical signal representations $\eta_{x}(t), \eta_{y}(t)$ of the two mutually orthogonal components of the electric field in a plane perpendicular to the direction of propagation

$$
\begin{aligned}
& \eta_{x}(t)=E_{x}(t)+\mathrm{i} \tilde{E}_{x}(t)=A_{x}(t) e^{i\left(u(t)+\beta_{x}(t)\right)}, \\
& \eta_{y}(t)=E_{y}(t)+\mathrm{i} \tilde{E}_{y}(t)=A_{y}(t) e^{i\left(u(t)+\beta_{y}(t)\right)},
\end{aligned}
$$

where, $\tilde{E}_{x}(t), \tilde{E}_{y}(t)$, are the Hilbert transforms of the real components of the electric field, and $u(t)=\bar{k} z-\bar{\omega} t$.

These two components can be arranged as the components of the following $2 \times 1$ complex vector

$$
\boldsymbol{\eta}(t)=\left(\begin{array}{l}
\eta_{1} \\
\eta_{2}
\end{array}\right)=e^{i\left(u(t)+\beta_{x}(t)\right)}\left(\begin{array}{l}
A_{x}(t) \\
A_{y}(t) e^{i\left(\beta_{y}(t)-\beta_{x}(t)\right)}
\end{array}\right),
$$

and, by avoiding the global phase factor (without physical meaning), the "instantaneous Jones vector" is defined as

$$
\varepsilon(t)=\left(\begin{array}{l}
A_{x}(t) \\
A_{y}(t) e^{i \delta_{y}(t)}
\end{array}\right)
$$

where $\delta_{y}(t)=\beta_{y}(t)-\beta_{x}(t)$ is the relative phase.

This vector includes all the information relative to the temporal evolution of the electric field. It is called "instantaneous" because the amplitudes and relative phase are time-dependent variables. In the case of a polychromatic wave, the instantaneous Jones vector has slow time dependence with respect to the coherence time, so that, for time intervals shorter than the coherence time, the polarization ellipse can be considered constant. For time intervals higher than the coherence time of the light wave, the instantaneous Jones vector can vary resulting in partially polarized light.

Thus, when the following quantities have not time dependence

$$
\frac{A_{y}(t)}{A_{x}(t)}=\text { constant, } \delta_{y}(t)=\text { constant }
$$

so that the electric field describes a stable well-defined ellipse (Fig. 1), the Jones vector is defined as [9]

$$
\varepsilon=\left(\begin{array}{l}
A_{x} \\
A_{y} e^{i \delta_{y}}
\end{array}\right)
$$

It should be noted that the conditions given by equation (6) are compatible with intensity fluctuations. In fact, totally polarized light maintains the azimuth and ellipticity of the polarization ellipse fixed whereas the size of the ellipse fluctuates resulting in a "mean intensity" over the measurement time. Moreover, slow time variations of the Jones vector with respect to the measurement time can be represented by this model [42]. 


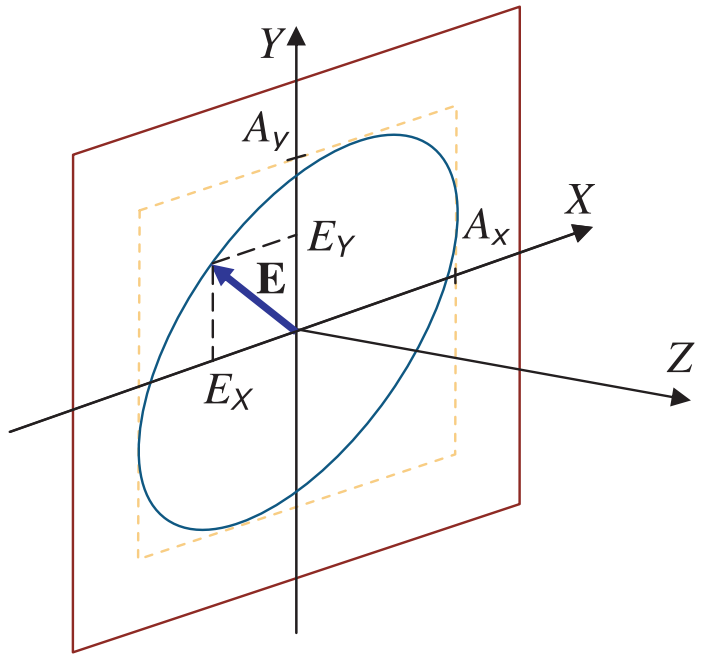

Fig. 1. (Color online) The polarization ellipse.

We see that the Jones vector provides a basic model for representing the state of polarization when light is totally polarized. The state of polarization of the coherent superposition of totally polarized light beams is given by the sum of the corresponding Jones vectors.

In the case of partially polarized light, the azimuth and (or) ellipticity of the polarization ellipse varies during a typical measurement time. In consequence, the treatment of partially polarized light requires a different mathematical model in order to take into account all the parameters that characterize completely the state of polarization.

\subsection{The coherency matrix}

The "coherency matrix" (or polarization matrix) $\boldsymbol{\Phi}[4$, $6,13,43-45]$ of a light beam contains all the measurable information about its state of polarization (including intensity). This Hermitian $2 \times 2$ matrix is defined as

$$
\boldsymbol{\Phi}=\left\langle\varepsilon(t) \otimes \varepsilon^{+}(t)\right\rangle=\left(\begin{array}{l}
\left\langle\varepsilon_{1}(t) \varepsilon_{1}^{*}(t)\right\rangle\left\langle\varepsilon_{1}(t) \varepsilon_{2}^{*}(t)\right\rangle \\
\left\langle\varepsilon_{2}(t) \varepsilon_{1}^{*}(t)\right\rangle\left\langle\varepsilon_{2}(t) \varepsilon_{2}^{*}(t)\right\rangle
\end{array}\right)
$$

where $\varepsilon$ is the instantaneous Jones vector whose two components are the analytic signals of the electric field of the wave; $\varepsilon^{+}$is the transposed conjugated vector of $\varepsilon ; \varepsilon_{i}^{*}$ represents the complex conjugate of $\varepsilon_{i} ; \otimes$ stands for the Kronecker product, and the brackets indicate time averaging over the measurement time

$$
\langle X(t)\rangle=\lim _{T \rightarrow \infty} \frac{1}{T} \int_{0}^{T} X(t) d t
$$

Therefore, $\boldsymbol{\Phi}$ is a covariance matrix whose elements are the second-order moments of the zero-mean analytic signals $\varepsilon_{i}(t) ; i=1,2$. Under the assumption that they are stationary and ergodic, the brackets can alternatively be considered as ensemble averaging of $\varepsilon \otimes \varepsilon^{+}$, where $\varepsilon$ are simple realizations.
Due to its statistical nature, $\boldsymbol{\Phi}$ is characterized by the fact that its two eigenvalues are non-negative. These two constraints are a complete set of necessary and sufficient conditions for a Hermitian matrix $\boldsymbol{\Phi}$ to be a coherency matrix, i.e. to represent a particular state of polarization of a light beam.

The statistical properties of $\mathbf{\Phi}$ appear explicitly when its elements are written in terms of the corresponding standard deviations $\sigma_{0}, \sigma_{1}$ and the complex degree of coherence $\mu$

$$
\mathbf{\Phi}=\left(\begin{array}{ll}
\sigma_{0}^{2} & \mu \sigma_{0} \sigma_{1} \\
\mu^{*} \sigma_{0} \sigma_{1} & \sigma_{1}^{2}
\end{array}\right) .
$$

As some authors have pointed out $[2,46]$ the normalized matrix

$$
\hat{\boldsymbol{\Phi}} \equiv \frac{\boldsymbol{\Phi}}{\operatorname{tr} \boldsymbol{\Phi}}
$$

is the corresponding density matrix, which contains information about the populations and coherences of the polarization states.

\subsection{The Stokes parameters}

The coherency matrix $\boldsymbol{\Phi}$ can be expressed as a linear expansion, with real coefficients, in the following basis, constituted by the three Pauli matrices plus the identity matrix

$$
\begin{array}{ll}
\boldsymbol{\sigma}_{0}=\left(\begin{array}{ll}
1 & 0 \\
0 & 1
\end{array}\right), & \boldsymbol{\sigma}_{1}=\left(\begin{array}{cc}
1 & 0 \\
0 & -1
\end{array}\right), \\
\boldsymbol{\sigma}_{2}=\left(\begin{array}{ll}
0 & 1 \\
1 & 0
\end{array}\right), & \boldsymbol{\sigma}_{3}=\left(\begin{array}{cc}
0 & -i \\
i & 0
\end{array}\right) .
\end{array}
$$

It should be noted that, although the habitual notations for the standard deviations $\left(\sigma_{0}, \sigma_{1}\right)$ and for the Pauli matrices $\left(\boldsymbol{\sigma}_{i}\right)$ have been preserved, this should not lead to confusion because $\sigma_{i}$ are matrices (bold), whereas the variances are scalar quantities.

This set of linearly independent matrices $\boldsymbol{\sigma}_{i}$ constitutes an adequate basis for the space of $2 \times 2$ Hermitian matrices. They are Hermitian $\boldsymbol{\sigma}_{i}=\boldsymbol{\sigma}_{i}^{+}$; trace-orthogonal $\operatorname{tr}\left(\boldsymbol{\sigma}_{i} \boldsymbol{\sigma}_{j}\right)=2 \delta_{i j}$, and satisfy $\boldsymbol{\sigma}_{i}^{2}=\mathbf{D}(1,1),[\mathbf{D}(1,1)$ being the $2 \times 2$ unity matrix]. Thus, these matrices are unitary and, except for $\sigma_{0}$, are traceless. This basis allows $\boldsymbol{\Phi}$ to be expressed as the following linear expansion $[4,47]$

$$
\boldsymbol{\Phi}=\frac{1}{2} \sum_{i=0}^{3} s_{i} \boldsymbol{\sigma}_{i}
$$

where the real coefficients $s_{i}$ are given by

$$
s_{i}=\operatorname{tr}\left(\boldsymbol{\Phi} \boldsymbol{\sigma}_{i}\right), \quad i=0,1,2,3 .
$$

The "Stokes parameters" $s_{0}, s_{1}, s_{2}, s_{3}$ are measurable quantities which satisfy the restrictions given by the inequalities

$$
s_{0} \geqslant 0, \quad s_{0}^{2} \geqslant s_{1}^{2}+s_{2}^{2}+s_{3}^{2},
$$

which are equivalent to the non-negativity of the coherency matrix $\boldsymbol{\Phi}$. 
These parameters, arranged as a $4 \times 1$ vector, constitute the "Stokes vector" s. Although in this section we will use the Stokes vector, we also introduce the following alternative notation in order to compare some expressions with others that will appear in later sections

$$
\mathbf{S}=\left(\begin{array}{ll}
s_{00} & s_{01} \\
s_{10} & s_{11}
\end{array}\right) \equiv\left(\begin{array}{ll}
s_{0} & s_{1} \\
s_{2} & s_{3}
\end{array}\right)
$$

\subsection{The purity criterion}

Let us consider now the Euclidean norms of $\mathbf{\Phi}$ and $\mathbf{S}$

$$
\begin{aligned}
& \|\mathbf{\Phi}\|_{2} \equiv\left[\sum_{i, j=0}^{1}\left|\phi_{i j}\right|^{2}\right]^{1 / 2}=\left[\operatorname{tr}\left(\boldsymbol{\Phi}^{+} \boldsymbol{\Phi}\right)\right]^{1 / 2}=\left[\operatorname{tr}\left(\boldsymbol{\Phi}^{2}\right)\right]^{1 / 2}, \\
& \|\mathbf{S}\|_{2} \equiv\left[\sum_{i=0}^{3} s_{i}^{2}\right]^{1 / 2}=\left[\operatorname{tr}\left(\mathbf{S}^{T} \mathbf{S}\right)\right]^{1 / 2} .
\end{aligned}
$$

Moreover, taking into account that $\boldsymbol{\Phi}$ is a positive semidefinite Hermitian matrix, we can define the following norm

$$
\|\boldsymbol{\Phi}\|_{0} \equiv \operatorname{tr} \boldsymbol{\Phi}=\|\sqrt{\boldsymbol{\Phi}}\|_{2}^{2} .
$$

It is easy to show that the above norms satisfy the following relations

$$
\begin{aligned}
\|\boldsymbol{\Phi}\|_{2}^{2} & =\frac{1}{2}\|\mathbf{S}\|_{2}^{2}, \\
\|\boldsymbol{\Phi}\|_{0} & =s_{0}, \\
\frac{1}{2}\|\boldsymbol{\Phi}\|_{0}^{2} & \leqslant\|\boldsymbol{\Phi}\|_{2}^{2} \leqslant\|\boldsymbol{\Phi}\|_{0}^{2} .
\end{aligned}
$$

For pure states, $\|\boldsymbol{\Phi}\|_{2}^{2}=\|\boldsymbol{\Phi}\|_{0}^{2}$. This equality constitutes an objective purity criterion, whereas the other limit $\|\boldsymbol{\Phi}\|_{2}^{2}=\frac{1}{2}\|\boldsymbol{\Phi}\|_{0}^{2}$ occurs in the case of unpolarized light (equiprobable mixture of states).

\subsection{Physical quantities characterizing the polarized light}

The Stokes vector can be written as

$$
\mathbf{s}=\left[\begin{array}{l}
I \\
I P \mathbf{u}
\end{array}\right]
$$

where the main physical magnitudes: (1) the intensity $I$; (2) the degree of polarization $P$; and (3) the unitary vector $\mathbf{u}$ that determines univocally the azimuth $\varphi(0 \leqslant \varphi<\pi)$ and the ellipticity angle $\chi(-\pi / 4 \leqslant \chi \leqslant \pi / 4)$ of the "average polarization ellipse", appear explicitly

$$
\begin{aligned}
& I=s_{0}, \quad P \equiv \frac{\left(s_{1}^{2}+s_{2}^{2}+s_{3}^{2}\right)^{1 / 2}}{s_{0}} \\
& \mathbf{u} \equiv \frac{1}{I P}\left(\begin{array}{c}
s_{1} \\
s_{2} \\
s_{3}
\end{array}\right)=\frac{1}{I P}\left(\begin{array}{c}
\cos 2 \chi \cos 2 \varphi \\
\cos 2 \chi \sin 2 \varphi \\
\sin 2 \chi
\end{array}\right) .
\end{aligned}
$$

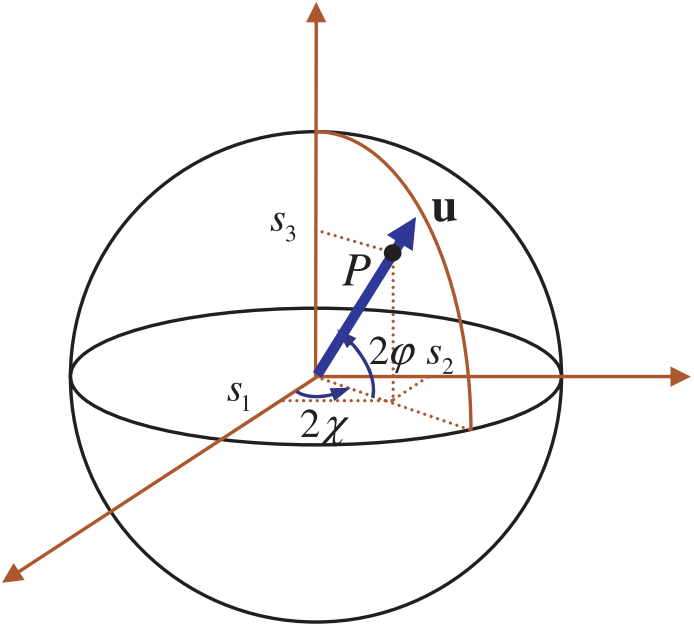

Fig. 2. (Color online) Poincaré sphere.

In the second-order approach, the Poincaré sphere (Fig. 2) provides a useful geometric representation of all possible states of polarization. Taking into account the characteristic properties of the Stokes parameters, they define a $4 \mathrm{D}$ cone, whose generatrix is the $s_{0}$-axis. The cut of this cone with the plane $s_{0}=1$ lead to the $3 \mathrm{D}$ representation given by the Poincaré sphere, which is a solid sphere of radius 1 where all states have an intensity equal to 1. Points on the surface represent totally polarized states (pure states) and points inside represent partially polarized states (mixed states). The origin represents secondorder unpolarized light.

\subsection{Decomposition of mixed states}

From equation (22), the following "trivial decomposition" of $\mathbf{s}$ as a convex sum of a pure state and an unpolarized state can be immediately obtained $[6,44]$

$$
\mathbf{s}=I\left[\begin{array}{c}
1 \\
P \mathbf{u}
\end{array}\right]=I\left(P\left[\begin{array}{l}
1 \\
\mathbf{u}
\end{array}\right]+(1-P)\left[\begin{array}{l}
1 \\
\mathbf{0}
\end{array}\right]\right) .
$$

Another alternative decomposition with different physical meaning is [44]

$$
\mathbf{s}=I\left[\begin{array}{c}
1 \\
P \mathbf{u}
\end{array}\right]=I\left(\frac{1+P}{2}\left[\begin{array}{l}
1 \\
\mathbf{u}
\end{array}\right]+\frac{1-P}{2}\left[\begin{array}{c}
1 \\
-\mathbf{u}
\end{array}\right]\right) .
$$

This "spectral decomposition", represented graphically in Figure 3, expresses a mixed state as a convex linear combination of two orthogonal pure states (their respective Jones vectors are orthogonal), which have the same direction in the Poincare sphere and are represented by antipodal points (these states have the same azimuth and their ellipticities differ in $\pi / 2$ ). The origin of the name spectral decomposition is derived from the fact that the above decomposition of a Stokes vector is equivalent to the spectral decomposition of its corresponding coherency matrix.

Nevertheless, given a mixed state, there exist infinite possibilities for decomposing it as a convex sum of two 


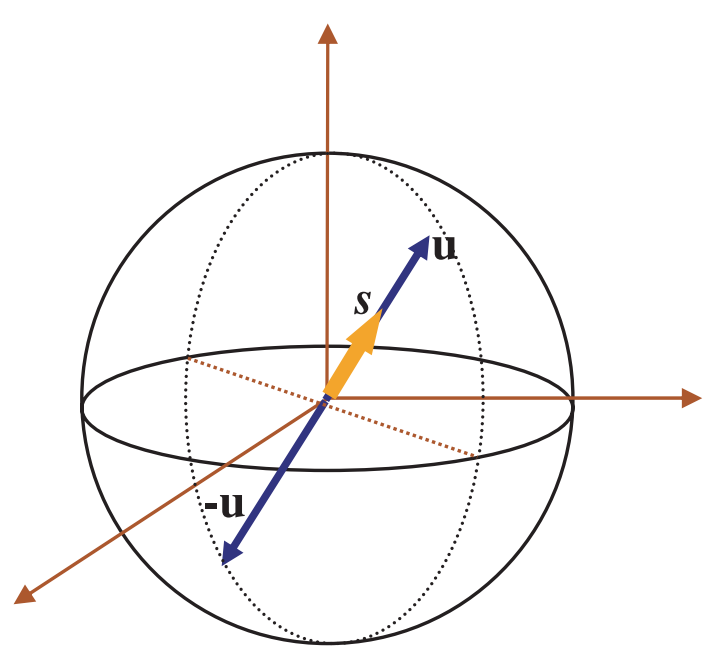

Fig. 3. (Color online) Spectral decomposition of a mixed state.

pure states

$$
\begin{aligned}
& \mathbf{s}=I\left[\begin{array}{c}
1 \\
P \mathbf{u}
\end{array}\right]=I(c \mathbf{x}+(1-c) \mathbf{y}) \\
& \mathbf{x} \equiv\left[\begin{array}{l}
1 \\
\mathbf{v}
\end{array}\right], \quad \mathbf{y} \equiv\left[\begin{array}{l}
1 \\
\mathbf{w}
\end{array}\right]
\end{aligned}
$$

where $\mathbf{v}, \mathbf{w}$ are linearly independent unitary vectors, and $0<c<1$.

Given a mixed state $\mathbf{s}$, any pure state $\mathbf{x}$ can be considered as a component. Once $\mathbf{x}$ is chosen, the coefficient $c$ and the remaining pure component $\mathbf{y}$ are univocally determined

$$
c=\frac{1-P^{2}}{2\left(1-P \mathbf{u}^{T} \mathbf{v}\right)}, \quad 0<c<1 ; \quad \mathbf{w}_{i}=\frac{P \mathbf{u}_{i}-c \mathbf{v}_{i}}{1-c} .
$$

Provided $\mathbf{s}$ is a mixed state, it is straightforward to show that the condition $0<c<1$ is always satisfied.

To illustrate this "arbitrary decomposition" by means of an explicit example, let us consider the mixed state $\mathbf{s}=(1,1 / 2,0,0)^{T}$ and let us chose the pure state $\mathbf{x}=$ $(1,0,1,0)^{T}$ as a component. We find that $c=3 / 8$ and $\mathbf{y}=(1,4 / 5,-3 / 5,0)^{T}$.

Although in a later section we will see a demonstration of the arbitrary decomposition of $3 \times 3$ coherency matrices, which can also be applied to $n \times n$ coherency matrices, we have considered it useful to include this demonstration in terms of Stokes vectors, because it provides a more intuitive view of this kind of decomposition.

The existence of different possible decompositions of a mixed state into pure states has particular importance due to its implications in the possible "target decompositions" used in radar polarimetry, where the spectral decomposition is usually considered to be the only way to achieve this [48].

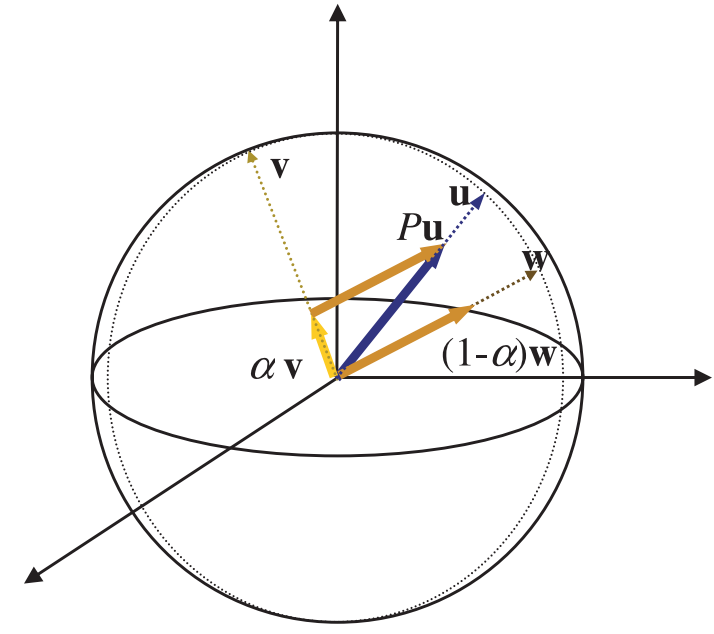

Fig. 4. (Color online) Arbitrary decomposition of a mixed state into a convex combination of two pure states.

Figure 4 illustrates the arbitrary decomposition by means of the corresponding representation in the Poincaré sphere.

\subsection{Physical invariants and degree of polarization}

Although the models of the Stokes vectors and the Poincaré sphere are particularly appropriate for representing the above decompositions, it is useful to return to the coherency matrix model because, as will be shown in later sections, this model can be generalized in order to represent the 3D states of polarization, as well as the polarimetric effects of linear media.

The $2 \times 2$ coherency matrix $\boldsymbol{\Phi}$ can be written in terms of the physical parameters $I, P, \chi, \varphi$

$$
\text { see equation (28) above }
$$

and, from this expression, it is straightforward to reproduce the trivial decomposition.

The diagonalization of $\boldsymbol{\Phi}$ leads to the spectral decomposition, expressed in terms of coherency matrices [44,49].

The quantities $I$ and $P$ are invariant in the sense that they remain unchanged under unitary transformations of the coherency matrix [50] and, hence, they are invariant with respect to changes of the reference system $X Y$. In fact, $I$ and $P$ are directly related with the eigenvalues of $\boldsymbol{\Phi}[44]$

$$
\begin{gathered}
\lambda_{0}=\frac{1}{2} I(1+P), \quad \lambda_{1}=\frac{1}{2} I(1-P) \\
I=\lambda_{0}+\lambda_{1}=\operatorname{tr} \boldsymbol{\Phi}, \quad P=\frac{\lambda_{0}-\lambda_{1}}{\operatorname{tr} \boldsymbol{\Phi}} .
\end{gathered}
$$

In agreement with the statistical nature of $\boldsymbol{\Phi}$, which is a covariance matrix (and, hence, is a positive semidefinite 
Hermitian matrix), $\lambda_{0}, \lambda_{1}$ are non-negative. Pure states are characterized by rank-1 polarization matrices (only one nonzero eigenvalue, $P=1$ ), whereas rank-2 polarization matrices correspond to mixed states $(P<1)$.

Appropriate expressions of $P$ that will be useful for future considerations are

$$
\begin{aligned}
& P_{(2)}=\left(\frac{2 \operatorname{tr}\left(\boldsymbol{\Phi}^{2}\right)}{(\operatorname{tr} \boldsymbol{\Phi})^{2}}-1\right)^{1 / 2}=\left(\frac{2\|\boldsymbol{\Phi}\|_{2}^{2}}{\|\boldsymbol{\Phi}\|_{0}^{2}}-1\right)^{1 / 2} \\
& P_{(2)}=\left(\frac{\sum_{i, j=0}^{1} s_{i j}^{2}}{s_{00}^{2}}-1\right)^{1 / 2}=\left(\frac{\operatorname{tr}\left(\mathbf{S}^{T} \mathbf{S}\right)}{s_{00}^{2}}-1\right)^{1 / 2}
\end{aligned}
$$

where the subscript (2) has been added in order to compare these expressions with other that will appear concerning higher order coherency matrices.

As another interesting way of studying $P$, Wolf, in a classic paper [6], showed that there always exist two orthogonal reference directions such that the degree of coherence $\mu$ reaches its maximum value, which coincides with $P$.

There are various random distributions which correspond to unpolarized light. As Ellis and Dogariu have shown [51], the measurement of the correlations of the Stokes parameters allows us to distinguish between these different types of unpolarized light. Moreover, for the case of non-Gaussian light, a quantitative determination of the degree of polarization of type-II unpolarized light by means of the distance between the polarization distribution and the uniform distribution associated with type-I unpolarized light, has been introduced by Luis [52].

\subsection{Polarization entropy}

It is opportune to consider now the concept of the von Neumann entropy $S$ applied to electromagnetic waves $[2,46,49,53-55]$, which, in terms of the density matrix $\hat{\boldsymbol{\Phi}}$ is defined as

$$
S=-\operatorname{tr}(\hat{\boldsymbol{\Phi}} \ln \hat{\boldsymbol{\Phi}}) .
$$

This quantity is a measure of the difference in the amount of information between a pure state and a mixed state (both with the same intensity) and can also be expressed as

$$
S=-\sum_{i=0}^{1}\left(\hat{\lambda}_{i} \ln \hat{\lambda}_{i}\right)
$$

where $\hat{\lambda}_{i}=\lambda_{i} / \operatorname{tr} \boldsymbol{\Phi}$ are the eigenvalues of $\hat{\boldsymbol{\Phi}}$. Thus, in the case of $2 \times 2$ coherency matrices, the von Neumann entropy can be written as a function of the degree of polarization $P[54]$

$$
\begin{aligned}
S=S_{(2)}(P) \equiv-\left\{\frac{1}{2}(\right. & 1+P) \ln \left[\frac{1}{2}(1+P)\right] \\
+ & \left.\frac{1}{2}(1-P) \ln \left[\frac{1}{2}(1-P)\right]\right\} .
\end{aligned}
$$

Therefore, $S_{(2)}$ is characterized univocally by $P$ and decreases monotonically as $P$ increases. The maximum $S_{(2)}=\ln 2$ corresponds to $P=0$, whereas the minimum $S_{(2)}=0$ is reached for $P=1$, (i.e. when light is totally polarized, regardless of its spectral profile) [2].

We observe that $S$ is dimensionless. Its relation with the concept of "specific radiation entropy" introduced by Plank, formulated explicitly by von Laue and extended by Barakat for $n$ pencils of radiation [53], has been studied by Barakat and Brosseau [54], who have expressed the normalized specific radiation entropy as the difference of two von Neumann entropies.

Moreover, from the von Neumann entropy, Brosseau and Bicout [56] have defined a "polarization temperature" which can be expressed as a monotonic decreasing function of the degree of polarization.

Although a detailed study of the different measures related with entropy and the relations among them falls outside the scope of this review, other relevant approaches to polarization entropy are briefly discussed in Sections 3.6. and 3.7 .

As we will see in later sections, the concept of "polarization entropy" [57], when related with the depolarizing effects of a material medium, is commonly used to analyze measurements and images [58] by remote sensing, by lidar detection and by synthetic aperture radar polarimetry (SAR Polarimetry) in order to detect spatial heterogeneity in a great variety of terrestrial and oceanic surface targets [59]. It also will be shown that a meaningful parameter defined as the degree of polarimetric purity can be used as a quantity alternative to entropy.

\subsection{Scope of the coherency matrix model and some extensions}

The Stokes parameters are defined through second-order moments of the field variables. Therefore, the statistical nature of light underlies in the mathematical description of the states of polarization of light, so that the study of the fluctuations of the field variables in different relevant cases is an important subject. For the cases of non-Gaussian probability distributions of these variables, higher order moments must be considered and, hence, the Stokes parameters do not provide a complete description of the state of polarization. Several authors have dealt with this matter, so that a significant amount of knowledge has been achieved [5,7,60-65]. A good compilation, including several contributions of the author, can be found in a book by Brosseau [2].

The measurement of the polarization properties of light beams with non-Gaussian spectral profiles should requires particular measurement arrangements involving interferometers and fast detectors.

Coherence properties have a close relation with polarization description, and several authors have dealt with this important subject [45,66-73].

A particular definition of Stokes parameters at the wavelet scale has been introduced by Castañeda [74]. 
These parameters take into account the correlation properties of the random electromagnetic field on the aperture plane.

A generalization of the Stokes parameters of a random electromagnetic beam has been introduced by Ellis and Dogariu [71] in the space-time domain and has also been studied by Korotkova and Wolf [75] in the space-frequency domain on the basis of the Wolf's unified theory of coherence and polarization [76]. Whereas the usual Stokes parameters depend on one spatial variable, these "two-point Stokes parameters", which can be defined in the spacefrequency domain from the cross-spectral density matrix that characterizes the correlations at two points, depend on two spatial variables and contain additional information about the coherence properties.

Another relevant approach to polarization description is the model of "spatial-angular Stokes parameters" developed by Luis $[77,78]$. Using Wigner functions [79], these parameters are introduced for generalized rays including spatial and angular dependence and allow their evolution during propagation to be considered.

Both the above-mentioned generalizations have their corresponding counterpart in Mueller representations, i.e. Mueller matrices transforming two-point Stokes parameters [80,81] and spatial-angular Mueller matrices transforming spatial-angular Stokes parameters [78].

The above paragraphs deal with a classical description of polarization. The Stokes parameters have been used traditionally to describe certain quantum systems [46,82]. Nevertheless, it should be noted that a quantum description of polarization, performed by means of replacing classical amplitudes by bosonic operators, results in some properties that cannot be fully described by the classical Stokes parameters [83]. Thus, for instance, the electric field vector never describes a definite ellipse (due to uncertainty relationships); there are states with $P=0$ that cannot be regarded as unpolarized, and $P$ does not reflect the lack of perfect polarization for any quantum state.

The quantum description of polarization states is necessary for the study and characterization of coherent, squeezed, number, and phase states, and for the design of experiments in order to show fundamental properties and applications of quantum theory such as entanglement, complementarity, information processing and cryptography, teleportation and Bell inequalities.

The characterization of states of polarization through probability distributions on the Poincaré sphere allows the definition of the quantum degree of polarization as the distance between the corresponding polarization distribution and the uniform distribution representing unpolarized light. This definition can be particularized as a trace distance between the coherency matrix and the identity matrix (unpolarized light), and is an important and open subject that provides results applicable to the description of quantum field states [83-86].

\section{3D Polarized light}

The two-dimensional formalism considered in the previous section is valid when the propagation direction of light is constant, which is the commonest physical situation of interest in polarimetry. In the most general case, the three components of the electric field vector of the light wave should be considered in order to describe the evolution of the end of the electric field vector (which determines the polarization state).

This section deals with the construction of a model for a general description of the polarization properties of light, applicable to waves whose direction of propagation is not stable in time. Thus, from the extension of the concept of the Jones vector, the $3 \mathrm{D}$ polarization matrix, as well as the 3D Stokes parameters, is defined. The main physical quantities characterizing the $3 \mathrm{D}$ states of polarization are obtained and analyzed, with special attention being given to the description of polarimetric purity by means of two invariant parameters.

\subsection{D Jones vector}

Let us consider a quasimonochromatic wave of arbitrary form, propagating in an isotropic medium, and let $\left(\mathbf{e}_{1}, \mathbf{e}_{2}, \mathbf{e}_{3}\right)$ be a reference basis of orthonormal vectors along the respective axes $X Y Z$. Given a point $\mathbf{r}$ in space, the analytic signals of the three components of the electric field of the electromagnetic wave can be arranged as the $3 \times 1$ complex vector

$$
\begin{aligned}
\boldsymbol{\eta}(t) & =\left(\begin{array}{l}
\eta_{1} \\
\eta_{2} \\
\eta_{3}
\end{array}\right)=\left(\begin{array}{c}
\eta_{x}(t) \\
\eta_{y}(t) \\
\eta_{z}(t)
\end{array}\right) \\
& =e^{i\left(u(t)+\beta_{x}(t)\right)}\left(\begin{array}{c}
A_{x}(t) \\
A_{y}(t) e^{i\left(\beta_{y}(t)-\beta_{x}(t)\right)} \\
A_{z}(t) e^{i\left(\beta_{z}(t)-\beta_{x}(t)\right)}
\end{array}\right)
\end{aligned}
$$

where $u(t)=\overline{\mathbf{k}} \cdot(\mathbf{r} /|\mathbf{r}|)-\bar{\omega} t, \overline{\mathbf{k}}$ being the mean wave-vector at the point $\mathbf{r}$.

The corresponding " $3 \mathrm{D}$ instantaneous Jones vector" is defined as

$$
\varepsilon(t)=\left(\begin{array}{c}
\varepsilon_{1}(t) \\
\varepsilon_{2}(t) \\
\varepsilon_{3}(t)
\end{array}\right)=\left(\begin{array}{c}
A_{x}(t) \\
A_{y}(t) e^{i \delta_{y}(t)} \\
A_{z}(t) e^{i \delta_{z}(t)}
\end{array}\right) .
$$

In this expression, the possible time dependence of amplitude and phases of the three components of the wave are indicated. Since only the phase differences have physical meaning, $\delta_{x}(t)$ has been taken as reference, so that a global phase factor has been avoided.

For totally-polarized sates, the following conditions are satisfied

$$
\begin{aligned}
& \frac{A_{y}(t)}{A_{x}(t)}=\text { constant }, \quad \frac{A_{z}(t)}{A_{x}(t)}=\text { constant }, \\
& \delta_{y}(t)=\text { constant }, \quad \delta_{z}(t)=\text { constant },
\end{aligned}
$$




$$
\mathbf{R}=\left(\begin{array}{ccc}
\left\langle A_{x}^{2}(t)\right\rangle & \left\langle A_{x}(t) A_{y}(t) e^{-i \delta_{y}(t)}\right\rangle & \left\langle A_{x}(t) A_{z}(t) e^{-i \delta_{z}(t)}\right\rangle \\
\left\langle A_{x}(t) A_{y}(t) e^{i \delta_{y}(t)}\right\rangle & \left\langle A_{y}^{2}(t)\right\rangle & \left\langle A_{y}(t) A_{z}(t) e^{i\left(\delta_{y}(t)-\delta_{z}(t)\right)}\right\rangle \\
\left\langle A_{x}(t) A_{z}(t) e^{i \delta_{z}(t)}\right\rangle\left\langle A_{y}(t) A_{z}(t) e^{-i\left(\delta_{y}(t)-\delta_{z}(t)\right)}\right\rangle & \left\langle A_{z}^{2}(t)\right\rangle
\end{array}\right) .
$$

and the generalized Jones vector is

$$
\varepsilon=\left(\begin{array}{l}
A_{x} \\
A_{y} e^{i \delta_{y}} \\
A_{z} e^{i \delta_{z}}
\end{array}\right)
$$

It is easy to verify that, in this case, the direction of propagation remains constant and the end of the electric field vector describes an ellipse perpendicular to the direction of propagation. The 2D model is then easily reproduced by taking the direction of propagation as the $Z$ reference axis, so that the third component of the 3D Jones vector is cancelled.

\subsection{D coherency matrix}

Several authors have dealt with the 3D description of the polarization state. From a chronological point of view, it is worth emphasizing the work of Roman [87], who defined a set of generalized Stokes parameters from a particular basis of $3 \times 3$ matrices. These matrices are not trace-orthogonal and, consequently, do not constitute the best option for building a basis. As Fano pointed out [46], a convenient basis for $n \times n$ coherency matrices is a set of Hermitian trace-orthogonal operators. Thus, for our purposes, an appropriate basis is that constituted by the $3 \times 3$ identity matrix together with the eight Gell-Mann matrices. We will see that this choice, based on the generators of the SU(3) group, is consistent with a generalization of the polarization algebra to $n \times n$ coherency matrices.

The so-called "coherency matrix" [6,43-45] or "polarization matrix", which contains all measurable information about the state of polarization (including intensity) of an electromagnetic wave, is defined as the following $3 \times 3$ Hermitian matrix

$$
\begin{aligned}
\mathbf{R} & =\left\langle\varepsilon(t) \otimes \varepsilon^{+}(t)\right\rangle \\
& =\left(\begin{array}{lll}
\left\langle\varepsilon_{1}(t) \varepsilon_{1}^{*}(t)\right\rangle & \left\langle\varepsilon_{1}(t) \varepsilon_{2}^{*}(t)\right\rangle & \left\langle\varepsilon_{1}(t) \varepsilon_{3}^{*}(t)\right\rangle \\
\left\langle\varepsilon_{2}(t) \varepsilon_{1}^{*}(t)\right\rangle & \left\langle\varepsilon_{2}(t) \varepsilon_{2}^{*}(t)\right\rangle & \left\langle\varepsilon_{2}(t) \varepsilon_{3}^{*}(t)\right\rangle \\
\left\langle\varepsilon_{3}(t) \varepsilon_{1}^{*}(t)\right\rangle & \left\langle\varepsilon_{3}(t) \varepsilon_{2}^{*}(t)\right\rangle & \left\langle\varepsilon_{3}(t) \varepsilon_{3}^{*}(t)\right\rangle
\end{array}\right),
\end{aligned}
$$

whose elements are the second-order moments of the zeromean analytic signals $\varepsilon_{i}(t) ; \quad i=1,2,3$, so that $\mathbf{R}$ is a covariance matrix and, therefore, $\mathbf{R}$ is completely characterized by the fact that it is a positive semidefinite Hermitian matrix [88].

The expression of $\mathbf{R}$ in terms of the amplitudes and relative phases of the field components is

$$
\text { see equation (40) above }
$$

As we have observed, $\mathbf{R}$ is characterized by the fact that its three eigenvalues are non-negative. These three constraints are a complete set of necessary and sufficient conditions for a Hermitian matrix $\mathbf{R}$ to be a " $3 \mathrm{D}$ coherency matrix", i.e. to represent a particular state of polarization of a light beam. An equivalent set of conditions is derived from the fact that the Hermitian matrix $\mathbf{R}$ has three nested non-negative principal minors [88].

The statistical properties of $\mathbf{R}$ arise clearly when its elements $r_{i j}(i, j=0,1,2)$ are written in terms of the corresponding standard deviations $\sigma_{i}$ and degrees of coherence $\mu_{i j}$,

$$
r_{i j} \equiv \mu_{i j} \sigma_{i} \sigma_{j}
$$

where $\sigma_{i}^{2}=\left\langle\varepsilon_{i} \varepsilon_{i}^{*}\right\rangle=r_{i i}, \mu_{i j}=r_{i j} / \sigma_{i} \sigma_{j}$, i.e.

$$
\mathbf{R}=\left(\begin{array}{ccc}
\sigma_{0}^{2} & \mu_{01} \sigma_{0} \sigma_{1} & \mu_{02} \sigma_{0} \sigma_{2} \\
\mu_{01}^{*} \sigma_{0} \sigma_{1} & \sigma_{1}^{2} & \mu_{12} \sigma_{1} \sigma_{2} \\
\mu_{02}^{*} \sigma_{0} \sigma_{2} & \mu_{12}^{*} \sigma_{1} \sigma_{2} & \sigma_{2}^{2}
\end{array}\right)
$$

From the non-negativity of three nested principal minors, we can write a set of necessary and sufficient explicit conditions for $\mathbf{R}$ to be a covariance matrix and, hence, to be a coherency matrix representing $3 \mathrm{D}$ states of polarization

$$
r_{00} \geqslant 0
$$

$$
1 \geqslant \rho_{01}
$$

$$
\begin{aligned}
\operatorname{det} \mathbf{R}=1+2 \rho_{01} \rho_{12} \rho_{02} \cos ( & \left.\beta_{01}+\beta_{12}-\beta_{02}\right) \\
& -\rho_{01}^{2}-\rho_{12}^{2}-\rho_{02}^{2} \geqslant 0
\end{aligned}
$$

where $\rho_{i j} \equiv\left|\mu_{i j}\right|, \mu_{i j} \equiv \rho e^{\beta_{i j}}$.

An interesting geometric interpretation of the $3 \mathrm{D}$ coherency matrix has been presented by Dennis [89] through the decomposition of $\mathbf{R}$ into a real symmetric positive definite matrix (interpreted as the moment of inertia of the ensemble, and represented by means of the corresponding ellipsoid) and a real axial vector (corresponding to the mean angular momentum of the ensemble). Another kind of representation of 3D states of polarization (but only valid for pure states), based on the Majorana sphere representation has been presented by Hannay [90] who has studied its relation with the Berry phase. Moreover, Saastamoinen and Tervo [91] and Ellis and Dogariu [92] have introduced geometric representations of the invariants of $\mathbf{R}$ in the eigenvalue space.

As a basis for the expansion of $\mathbf{R}$, let us now consider the following set of Hermitian, trace-orthogonal matrices constituted by the Gell-Mann matrices plus the identity 


$$
\begin{aligned}
& \boldsymbol{\omega}_{00} \equiv\left(\begin{array}{ccc}
1 & 0 & 0 \\
0 & 1 & 0 \\
0 & 0 & 1
\end{array}\right) \quad \boldsymbol{\omega}_{01} \equiv \sqrt{\frac{3}{2}}\left(\begin{array}{lll}
0 & 1 & 0 \\
1 & 0 & 0 \\
0 & 0 & 0
\end{array}\right) \quad \boldsymbol{\omega}_{02} \equiv \sqrt{\frac{3}{2}}\left(\begin{array}{lll}
0 & 0 & 1 \\
0 & 0 & 0 \\
1 & 0 & 0
\end{array}\right) \\
& \boldsymbol{\omega}_{10} \equiv \sqrt{\frac{3}{2}}\left(\begin{array}{ccc}
0 & -i & 0 \\
i & 0 & 0 \\
0 & 0 & 0
\end{array}\right) \quad \boldsymbol{\omega}_{11} \equiv \sqrt{\frac{3}{2}}\left(\begin{array}{ccc}
1 & 0 & 0 \\
0 & -1 & 0 \\
0 & 0 & 0
\end{array}\right) \quad \boldsymbol{\omega}_{12} \equiv \sqrt{\frac{3}{2}}\left(\begin{array}{lll}
0 & 0 & 0 \\
0 & 0 & 1 \\
0 & 1 & 0
\end{array}\right) \\
& \boldsymbol{\omega}_{20} \equiv \sqrt{\frac{3}{2}}\left(\begin{array}{ccc}
0 & 0 & -i \\
0 & 0 & 0 \\
i & 0 & 0
\end{array}\right) \quad \boldsymbol{\omega}_{21} \equiv \sqrt{\frac{3}{2}}\left(\begin{array}{ccc}
0 & 0 & 0 \\
0 & 0 & -i \\
0 & i & 0
\end{array}\right) \quad \boldsymbol{\omega}_{22} \equiv \frac{1}{\sqrt{2}}\left(\begin{array}{ccc}
1 & 0 & 0 \\
0 & 1 & 0 \\
0 & 0 & -2
\end{array}\right) \text {. }
\end{aligned}
$$

$$
\begin{aligned}
& q_{00} \equiv r_{00}+r_{11}+r_{22} \quad q_{01} \equiv \sqrt{\frac{3}{2}}\left(r_{01}+r_{10}\right) \quad q_{02} \equiv \sqrt{\frac{3}{2}}\left(r_{02}+r_{20}\right) \\
& q_{10} \equiv i \sqrt{\frac{3}{2}}\left(r_{01}-r_{10}\right) \quad q_{11} \equiv \sqrt{\frac{3}{2}}\left(r_{00}-r_{11}\right) \quad q_{12} \equiv \sqrt{\frac{3}{2}}\left(r_{12}+r_{21}\right) \\
& q_{20} \equiv i \sqrt{\frac{3}{2}}\left(r_{02}-r_{20}\right) \quad q_{21} \equiv i \sqrt{\frac{3}{2}}\left(r_{12}-r_{21}\right) q_{22} \equiv \frac{1}{\sqrt{2}}\left(r_{00}+r_{11}-2 r_{22}\right)
\end{aligned}
$$

$$
\mathbf{R}=\frac{1}{6}\left(\begin{array}{ccc}
\left(2 q_{00}+\sqrt{6} q_{11}+\sqrt{2} q_{22}\right) & \left(\sqrt{6} q_{01}-i \sqrt{6} q_{10}\right) & \left(\sqrt{6} q_{02}-i \sqrt{6} q_{20}\right) \\
\left(\sqrt{6} q_{01}+i \sqrt{6} q_{10}\right) & \left(2 q_{00}-\sqrt{6} q_{11}+\sqrt{2} q_{22}\right) & \left(\sqrt{6} q_{12}-i \sqrt{6} q_{21}\right) \\
\left(\sqrt{6} q_{02}+i \sqrt{6} q_{20}\right) & \left(\sqrt{6} q_{12}+i \sqrt{6} q_{21}\right) & \left(2 q_{00}-2 \sqrt{2} q_{22}\right)
\end{array}\right)
$$

matrix

$$
\text { see equation (44) above }
$$

The notation used for these matrices is justified for the sake of simplicity as well as to emphasize the symmetry in some future mathematical expressions.

As occurs with the basis used for the expansion of the $2 \times 2$ coherency matrices, the above matrices $\boldsymbol{\omega}_{i j}$ are Hermitian $\boldsymbol{\omega}_{i j}=\boldsymbol{\omega}_{i j}^{+}$; trace-orthogonal $\operatorname{tr}\left(\boldsymbol{\omega}_{i j} \boldsymbol{\omega}_{k l}\right)=3 \delta_{i k} \delta_{j l}$, and satisfy $\boldsymbol{\omega}_{i j}^{2}=\mathbf{D}(1,1,1)$. Thus, they are unitary and, except for the identity matrix $\boldsymbol{\omega}_{00}$, they are traceless.

The basis constituted by $\boldsymbol{\omega}_{i j}$ allows $\mathbf{R}$ to be expressed as the following linear combination [2]

$$
\mathbf{R}=\frac{1}{3} \sum_{i, j=0}^{2} q_{i j} \boldsymbol{\omega}_{i j}, \quad q_{i j}=\operatorname{tr}\left(\mathbf{R} \boldsymbol{\omega}_{i j}\right),
$$

where the nine real coefficients $q_{i j}$ can be properly called the "3D Stokes parameters".

In order to compare this expansion with that presented by other authors, the following observations should be taken into account:

- for the sake of symmetry and simplicity in the mathematical relations, the order chosen for the Gell-Mann matrices differs from that used by Gell-Mann and by several authors [93-95]. Except for the change $\boldsymbol{\omega}_{11} \leftrightarrow$ $\boldsymbol{\omega}_{22}$, the order coincides with the one used by Brosseau [2];

- the Gell-Mannn matrices have been normalized in order to ensure that their weight (Euclidean norm) coincide with that of the $3 \times 3$ identity matrix, which is also included in the basis. This choice differs from those used in the cited references, as well as in reference [96].

Obviously, these options have no physical consequences and do not affect the results expressed in terms of invariant quantities (i.e. derived from the eigenvalues of $\mathbf{R}$ ), but lead to different expressions for the 3D Stokes parameters and for their relations with other parameters.

As we will see, the normalization performed is consistent with a generalized model for the polarimetric properties of light and media based on $n$-dimensional coherency matrices.

\subsection{D Stokes parameters}

According to the definitions introduced in the previous section, the 3D Stokes parameters take the form

$$
\text { see equation (46) above }
$$

and the coherency matrix can be expressed in terms of $q_{i j}$ as follows

$$
\text { see equation (47) above }
$$

It is obvious that the Poincaré sphere representation is not applicable to $3 \mathrm{D}$ states of polarization. The constraining inequalities between $q_{i j}$ (derived from the non-negativity of the eigenvalues of $\mathbf{R}$ ) are of greater complexity than that of the $2 \mathrm{D}$ Stokes parameters. In fact, these restrictions involves, not only $\operatorname{tr} \mathbf{R}$ and $\operatorname{tr}\left(\mathbf{R}^{2}\right)$, but also $\operatorname{tr}\left(\mathbf{R}^{3}\right)$.

Now we examine the physical meaning of the 3D Stokes parameters. To do this, we first consider the reduction to 


$$
\mathbf{R}^{\prime}=\frac{1}{6}\left(\begin{array}{ccc}
\left(2 q_{00}^{\prime}+\sqrt{6} q_{11}^{\prime}+\sqrt{2} q_{22}^{\prime}\right) & \left(\sqrt{6} q_{01}^{\prime}-i \sqrt{6} q_{10}^{\prime}\right) & 0 \\
\left(\sqrt{6} q_{01}^{\prime}+i \sqrt{6} q_{10}^{\prime}\right) & \left(2 q_{00}^{\prime}-\sqrt{6} q_{11}^{\prime}+\sqrt{2} q_{22}^{\prime}\right) & 0 \\
0 & 0 & 0
\end{array}\right),
$$

the case of plane waves, which can be performed through a transformation of the reference axes $X Y Z$ to $X^{\prime} Y^{\prime} Z^{\prime}, Z^{\prime}$ being the propagation direction. The resulting coherency matrix $\mathbf{R}^{\prime}$ is

$$
\text { see equation (48) above }
$$

where the Stokes parameters can be interpreted by comparing them with the corresponding 2D Stokes parameters

$$
\begin{aligned}
s_{0} & =\frac{2}{3}\left(q_{00}^{\prime}+\frac{1}{\sqrt{2}} q_{22}^{\prime}\right), & s_{1} & =\sqrt{\frac{2}{3}} q_{11}^{\prime}, \\
s_{2} & =\sqrt{\frac{2}{3}} q_{01}^{\prime}, & s_{3} & =\sqrt{\frac{2}{3}} q_{10}^{\prime} .
\end{aligned}
$$

We see that, due to the particular normalization $\operatorname{tr}\left(\boldsymbol{\omega}_{i j} \boldsymbol{\omega}_{k l}\right)=3 \delta_{i k} \delta_{j l}$ chosen for $\boldsymbol{\omega}_{i j}$, and due to the intrinsic "asymmetry" of the Gell-Mann matrices, which have only two nonzero elements except for the diagonal matrix $\boldsymbol{\omega}_{22}$, the intensity $s_{0}$ includes a contribution from $q_{22}^{\prime}$. Thus, as Carozzi, Karlsson, and Bergman [93], and Setälä, Shevchenko, Kaivola and Friberg [94] have pointed out, in general, the parameter $q_{11}$ represents the predominance of the $X$ component $\left(q_{11}>0\right)$ or of the $Y$ component $\left(q_{11}<0\right)$. Attending to the projections of the field components on the $X Y$ plane, the value $q_{11}=0$ occurs, for example, in the cases of circularly polarized light and unpolarized light. Analogously, the parameters $q_{01}, q_{10}$ refer respectively to the predominance of projections on the positive $\left(q_{01}>0\right)$, or negative $\left(q_{01}<0\right)$, parts of the bisector axis of $X Y$ and of right handed $\left(q_{10}>0\right)$, or left handed $\left(q_{10}<0\right)$, circularly polarized light.

Analogous meanings correspond to the parameters $q_{02}, q_{20}$ (for projections on the $X Z$-plane), and $q_{12}, q_{21}$ (for projections on the $Y Z$-plane). Finally, the parameter $q_{22}$ represents the intensity in the $X Y$-plane additional to that in the $Z$-direction [94].

A particular analysis devoted to plane waves in the $3 \mathrm{D}$ spectral density tensor formalism is presented in reference [93], where the angles that determine the plane containing the polarization ellipse and the propagation direction are obtained from R. This analysis can also be applied to characterize the "spectral polarization ellipse" corresponding to each frequency contained in the light wave. In the time domain, and under the classic assumptions, the electric field describes the polarization ellipse, but, in general, both its shape and its plane vary with time. For the spectral density tensor, the dual pseudovector associated with its antisymmetric part is given by $i\left(-q_{21}, q_{12},-q_{20}\right)$, so that the real vector $\mathbf{v} \equiv\left(q_{21},-q_{12}, q_{20}\right)$ determines the plane of the polarization ellipse as well as the direction of propagation [93].

In the quantum model, these interpretations must be reconsidered because the polarization ellipse is not well defined [97], regardless of the existence of states of an essentially quantum nature.
As some authors have pointed out [98-100], the measurement of 3D Stokes parameters of near fields is an important subject for the study of coherent light diffracted by microstructures (producing subwavelength features) [99], fluorescence, multiphoton microscopy, imaging objects imbedded in dense scattering media, nanophotonics [100], etc. Dändliker et al. [99] have dealt with the measurement of the transverse component of the electric field by means of a heterodyne scanning probe optical microscope (heterodyne SPOM) and, more recently, Ellis and Dogariu [100] have suggested an original experimental setup for full 3D polarimetric measurements.

\subsection{Purity criterion for $3 \times 3$ coherency matrices}

The expression of the $3 \times 3$ coherency matrix $\mathbf{R}$ in terms of the statistical parameters, given by equation (42), shows that, as Ellis, Dogariu, Ponomarenko and Wolf have pointed out [101], total polarimetric purity corresponds to complete correlation between the field variables, so that the values of the modulus of the mutual degrees of coherence are equal to $1,\left|\mu_{i j}\right|=1$.

In order to express some relevant relations in terms of invariant metric parameters, let us now consider the Euclidean norms (also called Frobenius norms) of $\mathbf{R}$ and $\mathbf{Q} \equiv\left(q_{i j}\right)$

$$
\begin{aligned}
& \|\mathbf{R}\|_{2} \equiv\left(\sum_{i, j=0}^{2}\left|r_{i j}\right|^{2}\right)^{1 / 2}=\left[\operatorname{tr}\left(\mathbf{R}^{+} \mathbf{R}\right)\right]^{1 / 2}=\left[\operatorname{tr}\left(\mathbf{R}^{2}\right)\right]^{1 / 2} \\
& \|\mathbf{Q}\|_{2} \equiv\left(\sum_{i, j=0}^{2} q_{i j}^{2}\right)^{1 / 2}=\left[\operatorname{tr}\left(\mathbf{Q}^{T} \mathbf{Q}\right)\right]^{1 / 2}
\end{aligned}
$$

and, as in the $2 \mathrm{D}$ case, we define the following norm for $\mathbf{R}$

$$
\|\mathbf{R}\|_{0} \equiv \operatorname{tr} \mathbf{R}=\|\sqrt{\mathbf{R}}\|_{2}^{2}
$$

Since $\mathbf{R}$ is a positive semidefinite Hermitian matrix, it is easy to show that $\|\mathbf{R}\|_{0}$ satisfies all the conditions to be a norm. Furthermore, these norms satisfy the following relations

$$
\begin{aligned}
\|\mathbf{R}\|_{0} & =q_{00}, \\
\frac{1}{3}\|\mathbf{R}\|_{0}^{2} & \leqslant\|\mathbf{R}\|_{2}^{2} \leqslant\|\mathbf{R}\|_{0}^{2} .
\end{aligned}
$$

For pure states, the equality $\|\mathbf{R}\|_{2}^{2}=\|\mathbf{R}\|_{0}^{2}$ holds, which constitutes an objective purity criterion formulated in a symmetric manner with respect to that presented for the $2 \mathrm{D}$ case. The other limit $\|\mathbf{R}\|_{2}^{2}=\frac{1}{3}\|\mathbf{R}\|_{0}^{2}$ is reached in the case of unpolarized light (equiprobable mixture of states). 


\subsection{Decompositions of the coherency matrix}

In the following subsections we analyze different procedures for decomposing a coherency matrix $\mathbf{R}$ into a convex linear combination of particular coherency matrices. The spectral decomposition is based on the eigenvalue structure of $\mathbf{R}$ and consists of a set of pure components. The trivial decomposition is also based on the eigenvalue structure of $\mathbf{R}$ and consists of a pure component and a scaled set of unpolarized components. Finally, the arbitrary decomposition provides a general method for decomposing a mixed state into pure states and, hence, includes the spectral decomposition as a particular case.

\subsubsection{The spectral decomposition of the coherency matrix}

Since the coherency matrices are Hermitian positive semidefinite, they can be diagonalized through a unitary transformation. Therefore, the matrix $\mathbf{R}$ can be written as

$$
\mathbf{R}=\mathbf{U D}\left(\lambda_{0}, \lambda_{1}, \lambda_{2}\right) \mathbf{U}^{+}
$$

where $\mathbf{D}\left(\lambda_{0}, \lambda_{1}, \lambda_{1}\right)$ represents the diagonal matrix composed of the non-negative eigenvalues, ordered so that $0 \leqslant \lambda_{2} \leqslant \lambda_{1} \leqslant \lambda_{0}$. The columns $\mathbf{u}_{i} \quad(i=0,1,2)$ of the unitary matrix $\mathbf{U}$ are the respective eigenvectors.

Consequently, $\mathbf{R}$ can be expressed as the following convex linear combination of three rank-1 coherency matrices that represent respective pure states with the same intensity as $\mathbf{R}$

$$
\begin{aligned}
\mathbf{R}=\frac{\lambda_{0}}{\operatorname{tr} \mathbf{R}} \mathbf{U D}(\operatorname{tr} \mathbf{R}, 0,0) \mathbf{U}^{+} & +\frac{\lambda_{1}}{\operatorname{tr} \mathbf{R}} \mathbf{U D}(0, \operatorname{tr} \mathbf{R}, 0) \mathbf{U}^{+} \\
& +\frac{\lambda_{2}}{\operatorname{tr} \mathbf{R}} \mathbf{U D}(0,0, \operatorname{tr} \mathbf{R}) \mathbf{U}^{+}
\end{aligned}
$$

where each term in the sum is only affected by the corresponding eigenvector

$$
\mathbf{R}=\sum_{i=0}^{2} \frac{\lambda_{i}}{\operatorname{tr} \mathbf{R}}\left[(\operatorname{tr} \mathbf{R})\left(\mathbf{u}_{i} \otimes \mathbf{u}_{i}^{+}\right)\right]
$$

As in the 2D case, this spectral decomposition shows that any $3 \mathrm{D}$ polarization state can be considered as an incoherent superposition of three pure states with weights proportional to the eigenvalues of $\mathbf{R}$. The coherency matrix is a convex linear combination of the coherency matrices generated by their eigenvectors. It should be noted that, when one of the eigenvalues has a multiplicity higher than 1 , then the eigenvectors of the corresponding invariant subspace are not unique.

\subsubsection{The trivial decomposition of the coherency matrix}

The trivial decomposition of $\mathbf{R}$ cannot be performed in the form of a sum of a pure state and a 3D unpolarized state but, as we will see at the end of Section 3.6, a generalized trivial decomposition can be realized properly through a sum of a pure state, a 2D unpolarized state and a 3D unpolarized state. This agrees with the fact that the nine real independent parameters of $\mathbf{R}$ can be generated by the sum of a pure $3 \times 3$ coherency matrix (five real independent parameters), a 2 D-unpolarized $3 \times 3$ coherency matrix (three real independent parameters) and a 3D-unpolarized $3 \times 3$ coherency matrix (one real independent parameter). In general, pure $n \times n$ coherency matrices contain $2 n-1$ independent parameters whereas $m \mathrm{D}$-unpolarized $n \times n$ coherency matrices contain $2(n-m)+1$ independent parameters.

\subsubsection{The arbitrary decomposition of the coherency matrix}

As with the spectral decomposition, an arbitrary decomposition can also be applied to $3 \times 3$ coherency matrices

$$
\mathbf{R}=\sum_{i=0}^{2} \alpha_{i}\left[(\operatorname{tr} \mathbf{R})\left(\mathbf{v}_{i} \otimes \mathbf{v}_{i}^{+}\right)\right], \quad \sum_{i=0}^{2} \alpha_{i}=1,0 \leqslant \alpha_{i}
$$

where $\mathbf{v}_{i}$ are linearly independent unitary vectors. The number of pure components is equal to the rank of $\mathbf{R}$. The demonstration of the existence of this kind of decomposition for $n \times n$ coherency matrices, as well as the restrictions on $\mathbf{v}_{i}$, is dealt with in reference [102]. In our opinion this is a relevant result because provide all the ways for representing a mixed state as a superposition of pure states. Nevertheless, as we will see in Section 5.6.3, the application of this decomposition to coherency matrices representing material media requires that the mathematical expression of the superposition of pure states have the form of a convex sum. Thus, given the importance of this kind of decomposition and its potential application for scientific and industrial purposes we include here a new demonstration where $\mathbf{R}$ is expressed as a convex sum of pure components. It is straightforward to extend this demonstration to $n \times n$ coherency matrices.

Given a non-singular (rank-3) $3 \times 3$ coherency matrix $\mathbf{R}$, we consider equation (55) and write $\mathbf{R}$ as

$$
\begin{aligned}
& \mathbf{R}=\mathbf{C C}^{+}, \mathbf{C} \equiv \mathbf{U D}\left(\sqrt{\lambda_{0}}, \sqrt{\lambda_{1}}, \sqrt{\lambda_{2}}\right) \\
& \mathbf{C}^{+}=\mathbf{D}\left(\sqrt{\lambda_{0}}, \sqrt{\lambda_{1}}, \sqrt{\lambda_{2}}\right) \mathbf{U}^{+} .
\end{aligned}
$$

From successive steps we are going to decompose $\mathbf{R}$ into a linear convex combination of pure coherency matrices $\mathbf{A}^{(i)}$ satisfying $\operatorname{tr} \mathbf{A}^{(i)}=\operatorname{tr} \mathbf{R}$ (equal intensities before being affected by their respective weights in the convex sum).

First we observe that, given any rank-1 positive semidefinite Hermitian matrix $\mathbf{A}^{(2)}$, the matrix $(\mathbf{C})^{-1} \mathbf{A}^{(2)}\left(\mathbf{C}^{+}\right)^{-1}$ is a rank-1 Hermitian matrix, and there exist a unitary matrix $\mathbf{V}$ that diagonalizes it [103]

$$
\mathbf{V}^{+}\left[(\mathbf{C})^{-1} \mathbf{A}^{(2)}\left(\mathbf{C}^{+}\right)^{-1}\right] \mathbf{V}=\mathbf{D}\left(0,0, a_{2}\right), 0 \leqslant a_{2},
$$

and, consequently,

$$
\mathbf{A}^{(2)}=\mathbf{C V D}\left(0,0, a_{2}\right) \mathbf{V}^{+} \mathbf{C}^{+} .
$$


We introduce the following notations for $\mathbf{V}$ in order to use them in subsequent calculations

$$
\mathbf{V} \equiv\left(\begin{array}{lll}
v_{00} & v_{01} & v_{02} \\
v_{10} & v_{11} & v_{12} \\
v_{20} & v_{21} & v_{22}
\end{array}\right) \equiv\left(\mathbf{v}_{0}, \mathbf{v}_{1}, \mathbf{v}_{2}\right)
$$

where the column vectors $\mathbf{v}_{i} \equiv\left(v_{0 i}, v_{1 i}, v_{2 i}\right)^{T}$ are the orthogonal unitary eigenvectors of the matrix $\left[(\mathbf{C})^{-1} \mathbf{A}^{(2)}\left(\mathbf{C}^{+}\right)^{-1}\right]$.

Therefore, $\mathbf{A}^{(2)}$ can be written as

$$
\mathbf{A}^{(2)}=\mathbf{C}\left[a_{2}\left(\mathbf{v}_{2} \otimes \mathbf{v}_{2}^{+}\right)\right] \mathbf{C}^{+} .
$$

It is important to note that, whereas the eigenvector $\mathbf{v}_{2}$, corresponding to the nonzero eigenvalue $a_{2}$, is fixed by the particular election of $\mathbf{A}^{(2)}$, the other two orthogonal eigenvectors $\mathbf{v}_{0}, \mathbf{v}_{1}$, corresponding to the null subspace, can be chosen freely, provided they are orthogonal to $\mathbf{v}_{2}$.

Let us now consider the trace of $\mathbf{A}^{(2)}$, in order to determine the parameter $a_{2}$ from the condition $\operatorname{tr} \mathbf{A}^{(2)}=\operatorname{tr} \mathbf{R}$,

$$
\begin{aligned}
\operatorname{tr} \mathbf{A}^{(2)}= & \operatorname{tr}\left[\mathbf{C V D}\left(0,0, a_{2}\right) \mathbf{V}^{+} \mathbf{C}^{+}\right] \\
= & \operatorname{tr}\left\{\mathbf { U } \left[\mathbf{D}\left(\sqrt{\lambda_{0}}, \sqrt{\lambda_{1}}, \sqrt{\lambda_{2}}\right) \mathbf{V D}\left(0,0, a_{2}\right)\right.\right. \\
& \left.\left.\times \mathbf{V}^{+} \mathbf{D}\left(\sqrt{\lambda_{0}}, \sqrt{\lambda_{1}}, \sqrt{\lambda_{2}}\right)\right] \mathbf{U}^{+}\right\} \\
= & \operatorname{tr}\left[\mathbf{D}\left(\lambda_{0}, \lambda_{1}, \lambda_{2}\right) \mathbf{V D}\left(0,0, a_{2}\right) \mathbf{V}^{+}\right]=a_{2} l_{2},
\end{aligned}
$$

where

$$
\begin{aligned}
l_{2} & \equiv \operatorname{tr}\left[\mathbf{D}\left(\lambda_{0}, \lambda_{1}, \lambda_{2}\right) \mathbf{V D}(0,0,1) \mathbf{V}^{+}\right] \\
& =\operatorname{tr}\left\{\mathbf{D}\left(\lambda_{0}, \lambda_{1}, \lambda_{2}\right)\left[\mathbf{v}_{2} \otimes \mathbf{v}_{2}^{+}\right]\right\} \\
& =\lambda_{0}\left|v_{02}\right|^{2}+\lambda_{1}\left|v_{12}\right|^{2}+\lambda_{2}\left|v_{22}\right|^{2}, \quad l_{2}>0,
\end{aligned}
$$

and the trace condition $\operatorname{tr} \mathbf{A}^{(2)}=\operatorname{tr} \mathbf{R}$ leads to

$$
a_{2}=\frac{\operatorname{tr} \mathbf{R}}{l_{2}} \text {. }
$$

Moreover $\mathbf{R}$ can be expressed as

$$
\mathbf{R}=\mathbf{C C}^{+}=\mathbf{C}\left(\mathbf{V V}^{+}\right) \mathbf{C}^{+}=(\mathbf{C V}) \mathbf{D}(1,1,1)\left(\mathbf{V}^{+} \mathbf{C}^{+}\right)
$$

Thus, the simultaneous diagonalization of $\mathbf{A}^{(2)}$ and $\mathbf{R}$, allows us to perform the following subtraction

$$
\begin{aligned}
\mathbf{R}-\alpha_{2} \mathbf{A}^{(2)} & =\mathbf{C V}\left[\mathbf{D}(1,1,1)-\alpha_{2} \mathbf{D}\left(0,0, a_{2}\right)\right] \mathbf{V}^{+} \mathbf{C}^{+} \\
& =\mathbf{C V D}\left(1,1,1-\alpha_{2} a_{2}\right) \mathbf{V}^{+} \mathbf{C}^{+}
\end{aligned}
$$

We observe that, by taking $\alpha_{2}=1 / a_{2}=l_{2} / \operatorname{tr} \mathbf{R}$, the subtraction results in

$$
\mathbf{R}-\alpha_{2} \mathbf{A}^{(2)}=\mathbf{R}^{\prime}
$$

$$
\begin{aligned}
\mathbf{R}^{\prime} \equiv \mathbf{C V D}(1,1,0) \mathbf{V}^{+} \mathbf{C}^{+} \\
=\mathbf{C}\left[\left(\mathbf{v}_{0} \otimes \mathbf{v}_{0}^{+}\right)+\left(\mathbf{v}_{1} \otimes \mathbf{v}_{1}^{+}\right)\right] \mathbf{C}^{+},
\end{aligned}
$$

where $\mathbf{R}^{\prime}$ is a rank-2, positive semidefinite Hermitian matrix.

Let us now consider an arbitrary unitary matrix $\mathbf{V}^{\prime}$ of the form

$$
\mathbf{V}^{\prime} \equiv\left(\begin{array}{ccc}
v_{00}^{\prime} & v_{01}^{\prime} & 0 \\
v_{10}^{\prime} & v_{11}^{\prime} & 0 \\
0 & 0 & 1
\end{array}\right)
$$

This matrix $\mathbf{V}^{\prime}$ always satisfies the following matricial equation

$$
\mathbf{V}^{\prime} \mathbf{D}(1,1,0) \mathbf{V}^{\prime+}=\mathbf{D}(1,1,0),
$$

and, therefore

$$
\mathbf{R}^{\prime} \equiv \mathbf{C W D}(1,1,0) \mathbf{W}^{\prime+} \mathbf{C}^{+}, \quad \mathbf{W} \equiv \mathbf{V V}^{\prime},
$$

where, $\mathbf{W}$ is a unitary matrix with the form

$$
\mathbf{W} \equiv\left(\begin{array}{lll}
w_{00} & w_{01} & v_{02} \\
w_{10} & w_{11} & v_{12} \\
v_{20} & v_{21} & v_{22}
\end{array}\right) \equiv\left(\mathbf{w}_{0}, \mathbf{w}_{1}, \mathbf{v}_{2}\right)
$$

In order to find the class of rank-1 matrices susceptible to be diagonalized simultaneously with $\mathbf{R}^{\prime}$, we construct the matrix

$$
\mathbf{A}^{(1)}=\mathbf{C W D}\left(0, a_{1}, 0\right) \mathbf{W}^{+} \mathbf{C}^{+}
$$

so that

$$
\mathbf{A}^{(1)}=\mathbf{C}\left[a_{1}\left(\mathbf{w}_{1} \otimes \mathbf{w}_{1}^{+}\right)\right] \mathbf{C}^{+},
$$

where, as we have observed, the vector $\mathbf{w}_{1}$ can be chosen arbitrarily in the subspace orthogonal to $\mathbf{v}_{2}$.

The trace of $\mathbf{A}^{(1)}$ is given by

$$
\operatorname{tr} \mathbf{A}^{(1)}=\operatorname{tr}\left[\mathbf{C W D}\left(0, a_{1}, 0\right) \mathbf{W}^{+} \mathbf{C}^{+}\right]=a_{1} l_{1},
$$

where

$$
\begin{aligned}
l_{1} & \equiv \operatorname{tr}\left[\mathbf{D}\left(\lambda_{0}, \lambda_{1}, \lambda_{2}\right) \mathbf{W D}(0,1,0) \mathbf{W}^{+}\right] \\
& =\operatorname{tr}\left\{\mathbf{D}\left(\lambda_{0}, \lambda_{1}, \lambda_{2}\right)\left[\mathbf{w}_{1} \otimes \mathbf{w}_{1}^{+}\right]\right\} \\
& =\lambda_{0}\left|w_{01}\right|^{2}+\lambda_{1}\left|w_{11}\right|^{2}+\lambda_{2}\left|w_{21}\right|^{2}, \quad l_{1}>0 .
\end{aligned}
$$

Due to the non-uniqueness of the eigenvectors corresponding to the subspace given by the degenerate eigenvalue 1 , the coherency matrix $\mathbf{R}^{\prime}$ can be written as

$$
\begin{aligned}
\mathbf{R}^{\prime} & =\mathbf{C W D}(1,1,0) \mathbf{W}^{+} \mathbf{C}^{+} \\
& =\mathbf{C}\left[\left(\mathbf{w}_{0} \otimes \mathbf{w}_{0}^{+}\right)+\left(\mathbf{w}_{1} \otimes \mathbf{w}_{1}^{+}\right)\right] \mathbf{C}^{+} .
\end{aligned}
$$

Thus, the simultaneous diagonalization of $\mathbf{A}^{(1)}$ and $\mathbf{R}^{\prime}$ allows us to perform the following subtraction

$$
\mathbf{R}^{\prime}-\alpha_{1} \mathbf{A}^{(1)}=\mathbf{C W}\left[\mathbf{D}\left(1,1-\alpha_{1} a_{1}, 0\right)\right] \mathbf{W}^{+} \mathbf{C}^{+},
$$

and, by choosing $a_{1}=\operatorname{tr} \mathbf{R} / l_{1}$ and $\alpha_{1}=1 / a_{1}=l_{1} / \operatorname{tr} \mathbf{R}$, we obtain

$$
\mathbf{R}^{\prime \prime}=\mathbf{R}^{\prime}-\alpha_{1} \mathbf{A}^{(1)}=\mathbf{C W D}(1,0,0) \mathbf{W}^{+} \mathbf{C}^{+} .
$$

The last pure component is fully determined by the previous choices of $\mathbf{A}^{(2)}$ and $\mathbf{A}^{(1)}$ and can be written as

$$
\begin{aligned}
\mathbf{A}^{(0)} & =a_{0} \mathbf{R}^{\prime \prime}=\mathbf{C W D}\left(a_{0}, 0,0\right) \mathbf{W}^{+} \mathbf{C}^{+} \\
& =\mathbf{C}\left[a_{0}\left(\mathbf{w}_{0} \otimes \mathbf{w}_{0}^{+}\right)\right] \mathbf{C}^{+},
\end{aligned}
$$


so that its trace is

$$
\operatorname{tr} \mathbf{A}^{(0)}=a_{0} l_{0}
$$

where

$$
\begin{aligned}
l_{0} & \equiv \operatorname{tr}\left[\mathbf{D}\left(\lambda_{0}, \lambda_{1}, \lambda_{2}\right) \mathbf{W D}(1,0,0) \mathbf{W}^{+}\right] \\
& =\operatorname{tr}\left\{\mathbf{D}\left(\lambda_{0}, \lambda_{1}, \lambda_{2}\right)\left[\mathbf{w}_{0} \otimes \mathbf{w}_{0}^{+}\right]\right\} \\
& =\lambda_{0}\left|w_{00}\right|^{2}+\lambda_{1}\left|w_{10}\right|^{2}+\lambda_{2}\left|w_{20}\right|^{2}, \quad l_{0}>0 .
\end{aligned}
$$

Analogously to the previous steps, we chose $a_{0}$ and $\alpha_{0}$ satisfying $a_{0}=\operatorname{tr} \mathbf{R} / l_{0}$ and $\alpha_{0}=1 / a_{0}=l_{0} / \operatorname{tr} \mathbf{R}$. that

From the properties of the unitary matrix $\mathbf{W}$ we see

$$
\sum_{i=0}^{2} l_{i}=\operatorname{tr} \mathbf{R}
$$

which is the condition for the following expansion to be a convex sum

$$
\begin{aligned}
\mathbf{R} & =\sum_{i=0}^{2} \alpha_{i} \mathbf{A}^{(i)}, \\
\mathbf{A}^{(i)} & =\left(\mathbf{A}^{(i)}\right)^{+}, \\
\sum_{i=0}^{2} \alpha_{i} & =\sum_{i=0}^{2} \frac{l_{i}}{\operatorname{tr} \mathbf{R}}=1, \\
\operatorname{tr} \mathbf{A}^{(i)} & =\operatorname{tr} \mathbf{R}, \quad \operatorname{rank}\left(\mathbf{A}^{(i)}\right)=1 .
\end{aligned}
$$

For the case $\operatorname{rank}(\mathbf{R})=2$ the same procedure can be applied by starting from the expression of the coherency matrix $\mathbf{R}^{\prime}$.

This result can be interpreted as follows: any mixed state of $3 \mathrm{D}$ polarized light is physically equivalent to an arbitrary incoherent superposition of up to three independent pure sates. When $\operatorname{rank}(\mathbf{R})=3$, any pure state can be considered as a component and, once chosen, the election of the second component is not completely arbitrary, whereas the third component is fully determined by the previous choices. When $\operatorname{rank}(\mathbf{R})=2$, the direction of propagation is fixed, and the mixed state corresponds to a $2 \mathrm{D}$ state of partially polarized light. This agrees with the arbitrary decomposition seen in the section devoted to $2 \mathrm{D}$ polarized light.

Obviously, the spectral decomposition is a particular case of the arbitrary decomposition. Moreover, we see that any mixed state with can be expressed as a convex sum of an arbitrary pure state and a corresponding mixed state.

As we will show, this new result is also relevant when applied to coherency matrices representing the polarimetric properties of material media.

\subsection{Polarimetric purity of $3 \times 3$ coherency matrices}

A first proper definition of the 3D degree of polarization was presented by Samson in 1973 [104] within the scope of geophysical studies of ultra-low frequency magnetic fields.
This result was also obtained by Barakat by formulating the degree of polarization in terms of scalar invariants of the coherency matrix [53]. Nevertheless, the study of the $3 \mathrm{D}$ degree of polarization has recently attracted attention due to the advances in optical nanotechnologies and from the necessity of understanding polarization phenomena in fluctuating near fields and evanescent waves.

A complete formulation of the purity of the $3 \mathrm{D}$ states of polarization based on two relative differences of the eigenvalues of the coherency matrix (indices of purity) has been introduced by Gil, Correas, Melero and Ferreira [41]. More recently, Ellis, Dogariu, Ponomarenko and Wolf [105] have also presented two parameters based on two relative differences of the eigenvalues of the coherency matrix and, from this result, Hioe [106] has introduced a parameter called the "degree of isotropy". We refer also to the relevant approaches obtained by Réfrégier, Roche and Goudail [107] who have emphasized the necessity of three quantities to characterize the invariant properties of $3 \times 3$ coherency matrices and have shown that, for light with Gaussian fluctuations, a set of three invariant parameters is enough to characterize the "polarimetric contrast", so that different sets of three invariant parameters defined from the 3D coherency matrix have been considered, as well as their relations with the different "degrees of polarization" defined by Barakat [53] and by Samson [104]. Réfrégier and Goudail have also proposed the Kullback relative entropy as an invariant parameter that, together with the intensity and the 3D degree of polarization, completes a set of invariant parameters [108].

Another purity parameter has recently been introduced by Dennis [109] by means of averaging the 3D state of polarization due to a dipole over all scattering directions, which leads to a purity measure which is not a unitary invariant of the coherency matrix.

Particularly relevant contributions concerning the concept of the 3D degree of polarization have been presented by Setälä, Shevchenko, Kaivola and Friberg [94,98] and Ellis and Dogariu [92].

Quantum and classical approaches to the 3D degree of polarization concept, defining it as a distance between distributions [83] and between correlation matrices have been presented by Luis $[95,110]$. The connection between the degree of polarization and the effective degree of coherence has been studied by Vahimaa and Tervo [111].

The 3D "degree of polarization" can be defined as $[53,98,104]$

$$
P_{(3)}=\left|\left[\frac{1}{2}\left(\frac{3 \operatorname{tr}\left(\mathbf{R}^{2}\right)}{(\operatorname{tr} \mathbf{R})^{2}}-1\right)\right]^{1 / 2}\right| .
$$

This invariant non-dimensional parameter is limited to the interval $0 \leqslant P_{(3)} \leqslant 1$, in such a manner that $P_{(3)}=1$ corresponds to the case that $\mathbf{R}$ has only one nonzero eigenvalue (total polarimetric purity: the direction of propagation is constant and the electric field describes a welldefined polarization ellipse), whereas $P_{(3)}=0$ is reached when the three eigenvalues of $\mathbf{R}$ are equal (equiprobable 
mixture of states and zero correlation between the electric field components).

For reasons that will be made clear below, we prefer using the term "degree of purity" (which obviously refers to polarimetric purity), rather than "degree of polarization", for $P_{(3)}$.

The expression of $P_{(3)}$ in function of the 3D Stokes parameters is

$$
\begin{aligned}
P_{(3)} & =\left|\left[\frac{1}{2}\left(\frac{\sum_{i, j=0}^{2} q_{i}^{2}}{q_{00}^{2}}-1\right)\right]^{1 / 2}\right| \\
& =\left|\left[\frac{1}{2}\left(\frac{\operatorname{tr}\left(\mathbf{Q}^{T} \mathbf{Q}\right)}{q_{00}^{2}}-1\right)\right]^{1 / 2}\right|
\end{aligned}
$$

and, in terms of both norms of $\mathbf{R}$

$$
P_{(3)}=\left|\left[\frac{1}{2}\left(\frac{3\|\mathbf{R}\|_{2}^{2}}{\|\mathbf{R}\|_{0}^{2}}-1\right)\right]^{1 / 2}\right| .
$$

As Setälä et al. have pointed out $[94,98], P_{(3)}$ takes into account not only the purity of the mean polarization ellipse, but also the stability of the plane that contains the instantaneous components of the electric field of the wave. Thus, for unpolarized light whose propagation direction remains fixed, $P_{(2)}=0$ whereas $P_{(3)}=1 / 2$. It is clear that in the $3 \mathrm{D}$ description of polarization, new relevant quantities and peculiar properties arise that do not exist in the 2D model. Therefore, the existence of three eigenvalues leads to the fact that, unlike the $2 \mathrm{D}$ model, the overall degree of purity $P_{(3)}$ does not provide complete information of the polarimetric purity properties. In order to find adequate invariant non-dimensional quantities that provide a complete description of the purity of 3D polarization states, we return to $P_{(2)}$ and observe that it can be defined as a relative difference between the two eigenvalues, so that $0 \leqslant P_{(2)} \leqslant 1$. Thus, in the light of the structure of the algebraic expressions of the eigenvalues of $\mathbf{R}$, and by inspecting the various relative differences between them, we see that a convenient pair of "indices of purity" is defined as [41]

$$
P_{1}=\frac{\lambda_{0}-\lambda_{1}}{\operatorname{tr} \mathbf{R}}, \quad P_{2}=\frac{\lambda_{0}+\lambda_{1}-2 \lambda_{2}}{\operatorname{tr} \mathbf{R}} .
$$

These non-dimensional quantities are restricted by the following limits

$$
0 \leqslant P_{1} \leqslant P_{2} \leqslant 1
$$

From the above equations, the following quadratic relation between $P_{(3)}$ and the two indices of purity $P_{1}, P_{2}$ is derived

$$
P_{(3)}^{2}=\frac{1}{4}\left(3 P_{1}^{2}+P_{2}^{2}\right) \text {. }
$$

Another interesting expression of $P_{(3)}$, as a homogeneous quadratic measure of all the relative differences between

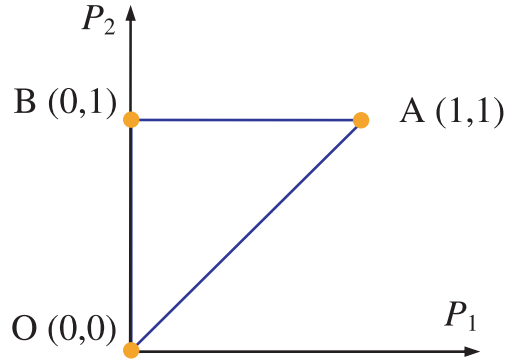

Fig. 5. (Color online) Feasible region for $P_{1}, P_{2}$ in the purity space.

the eigenvalues, is the following

$$
P_{(3)}^{2}=\frac{1}{2} \sum_{\substack{i, j=0 \\ i<j}}^{2} p_{i j}^{2}, \quad p_{i j} \equiv \frac{\lambda_{i}-\lambda_{j}}{\operatorname{tr} \mathbf{R}} .
$$

Thus, the two indices of purity provide complete information about the polarimetric purity of the corresponding polarization state. This enhanced description based on invariant, non-dimensional parameters with values restricted to the range between 0 and 1, has special significance from a physical point of view.

The physically feasible region in the purity space $P_{1}, P_{2}$ is shown in Figure 5.

As we will see in the case analysis presented below, $P_{1}$ maintain its meaning of " $2 \mathrm{D}$ degree of polarization" whereas $P_{2}$ have the following properties:

- if $P_{1}=0$ (fully random polarization ellipse), then $0 \leqslant P_{2} \leqslant 1$. We see that the only possible contribution to purity of $P_{2}$ is related with the stability of the propagation direction;

- if $P_{1}=1$ (pure $2 \mathrm{D}$ state), then $P_{2}=1$ and $P_{(3)}=1$ (3D pure state). This agrees with the fact that a $2 \mathrm{D}$ pure state is a $3 \mathrm{D}$ pure state with fixed direction of propagation;

- if $P_{2}=0$, then $P_{1}=0$ and $P_{(3)}=0$ (3D unpolarized state). This agrees with the fact that a random propagation direction entails a random polarization ellipse.

These arguments indicate that $P_{2}$ is a measure of the degree of stability of the propagation direction of the wave.

To provide a good understanding of the physical meaning of the indices of purity, we analyze different states represented in the corresponding feasible region.

(a) $P_{1}=0,0<P_{2}<1$

$0<P_{(3)}<1 / 2 ; \quad \lambda_{0}=\lambda_{1}>\lambda_{2}>0$. The system is equivalent to an incoherent combination of three pure contributions. The more significant two have equal intensities $\lambda_{0}=\lambda_{1}>\lambda_{2}$. These states are represented in the edge $\mathrm{OB}$ (vertices $\mathrm{O}$ and $\mathrm{B}$ excluded) and correspond to light whose direction of propagation fluctuates $\left(P_{2}<1\right)$ with a predominance of an "average direction of propagation", whereas the "instantaneous polarization ellipse" is fully random $\left(P_{1}=0\right)$. 
(b) $0 \leqslant P_{1} \leqslant 1, P_{2}=1$

$1 / 2 \leqslant P_{(3)} \leqslant 1 ; \lambda_{0} \geqslant \lambda_{1} \geqslant 0, \lambda_{0}>0, \lambda_{2}=0$. The system is composed of two incoherent beams with orthogonal states of polarization and superposed in the same fixed direction of propagation, so that this case corresponds to 2D partially polarized light. These states are represented in the edge BA. $P_{1}$ is the $2 \mathrm{D}$ degree of polarization $P_{(2)}$, whereas the constancy of the propagation direction results in a contribution of $1 / 2$ to the value of $P_{(3)}$. Therefore, it is clear that $P_{2}$ refers directly to the stability of the propagation direction.

(b.1) $P_{1}=P_{2}=1$

$P_{(3)}=1 ; \quad \lambda_{0}>0, \lambda_{1}=\lambda_{2}=0$. The vertex A represents this state of totally polarized light, i.e. light with fixed direction of propagation and stable mean polarization ellipse (in short times only the intensity fluctuates; whereas the azimuth $\chi$ and the ellipticity $\varphi$ do not).

(b.2) $P_{1}=0, P_{2}=1$

$P_{(3)}=1 / 2 ; \quad \lambda_{0}=\lambda_{1}>0, \lambda_{2}=0$. The point $\mathrm{B}$ represents this state of $2 \mathrm{D}$ unpolarized light, i.e. plane wave with fixed direction of propagation and equiprobable statistical contributions of the two orthogonally polarized incoherent components.

(c) $0<P_{2}=P_{1}<1$

$P_{(3)}=P_{2}=P_{1} ; \quad \lambda_{0}>\lambda_{1}\left(=\lambda_{2}\right)>0$. The propagation direction and the instantaneous polarization ellipse fluctuate with the same randomness $P_{2}=P_{1}$. These states are represented in the segment OA (vertices $\mathrm{O}$, A excluded). $P_{1}$ has a maximum value compatible with $P_{2}$.

(d) $P_{1}=P_{2}=0$

$P_{(3)}=0 ; \quad \lambda_{0}=\lambda_{1}=\lambda_{2}>0$. This state is represented by the vertex $\mathrm{O}$ and corresponds to a fully random $3 \mathrm{D}$ state of polarization. No predominance exists in the direction of propagation and in the polarization ellipse. The only nonzero 3D Stokes parameter is $q_{00}$. This is an extreme physical situation which can be generated by intersecting three orthogonal light beams with the same intensity. It is very important to note that the inequality $P_{1} \leqslant P_{2}$ indicates that the value of $P_{1}$ is limited by the value of $P_{2}$. This result is consequent with the expected physical behavior. In this case, fully random direction of propagation implies zero purity of the polarization ellipse.

(e) $0<P_{1}<P_{2}<1$

$0<P_{(3)}<1 ; \quad \lambda_{0}>\lambda_{1}>\lambda_{2}>0$. These states are represented by points inside the triangle OBA and exhibit partially random fluctuations of the direction of propagation and of the instantaneous polarization ellipse (with different randomness $P_{1} \neq P_{2}$ ). It should be noted again that the purity of the fluctuations of the polarization ellipse $\left(P_{1}\right)$ can never exceed the purity of the fluctuations of the direction of propagation $\left(P_{2}\right)$.

The above analysis leads us to propose the following names for the indices of purity, $P_{1}$ : degree of polarization; $P_{2}$ : degree of directionality, and $P_{(3)}$ : degree of purity. The names are consistent with the concepts underlying these relevant quantities. We also observe that this disquisition agrees with the conclusions of the study of Ellis and Dogariu concerning the concept of degree of polarization and the other invariant quantities describing the purity of a polarization state [92]. In fact, in the light of the previous analysis, we can consider the following decomposition proposed by Holm and Barnes [112], which has also been considered by Cloude and Pottier [113] and by Ellis and Dogariu [92]

$$
\begin{aligned}
& \mathbf{R}=\mathbf{U D}\left(\lambda_{0}, \lambda_{1}, \lambda_{2}\right) \mathbf{U}^{+}=\mathbf{A}+\mathbf{B}+\mathbf{C} \\
& \mathbf{A} \equiv \mathbf{U D}\left(\lambda_{0}-\lambda_{1}, 0,0\right) \mathbf{U}^{+}, \\
& \mathbf{B} \equiv \mathbf{U D}\left(\lambda_{1}-\lambda_{2}, \lambda_{1}-\lambda_{2}, 0\right) \mathbf{U}^{+}, \\
& \mathbf{C} \equiv \mathbf{U D}\left(\lambda_{2}, \lambda_{2}, \lambda_{2}\right) \mathbf{U}^{+}
\end{aligned}
$$

where the coherency matrix $\mathbf{R}$ is expressed as a sum of the following coherency matrices: $\mathbf{A}$, which represents a 2D pure state (vertex A, case (b.1)); $\mathbf{B}$, which represents a $2 \mathrm{D}$ unpolarized state (vertex B, case (b.2)), and $\mathbf{C}$, which represents a $3 \mathrm{D}$ unpolarized state (vertex $\mathrm{O}$, case $(\mathrm{d})$ ). Provided $P_{2}=1$ (i.e. $\lambda_{2}=0$ ), this decomposition results in the $2 \mathrm{D}$ trivial decomposition.

This result can be expressed as follows: "any 3D polarization state can be considered as a superposition of three polarization states: a pure state, a $2 D$ unpolarized state and a 3D unpolarized state" [92].

Therefore, the decomposition of a mixed state into a pure state and an unpolarized state must be performed in this scaled way, where the unpolarized term is composed of a $2 \mathrm{D}$ unpolarized state and a $3 \mathrm{D}$ unpolarized state. This point of view definitely clarifies the fundamental meaning of the generalized trivial decomposition. We consequently propose the name "3D trivial decomposition" for the decomposition given by equation (93).

Moreover, it is interesting to express this decomposition by means of a linear convex combination of light states (with equal intensities before being affected by their respective weights in the convex sum)

$$
\begin{aligned}
\mathbf{R} & =\frac{\lambda_{0}-\lambda_{1}}{\operatorname{tr} \mathbf{R}} \mathbf{A}^{(0)}+2 \frac{\lambda_{1}-\lambda_{2}}{\operatorname{tr} \mathbf{R}} \mathbf{B}^{(1)}+3 \frac{\lambda_{2}}{\operatorname{tr} \mathbf{R}} \mathbf{B}^{(2)} \\
\mathbf{A}^{(0)} & \equiv \operatorname{tr} \mathbf{R}\left[\mathbf{U D}(1,0,0) \mathbf{U}^{+}\right] \\
\mathbf{B}^{(1)} & \equiv \frac{1}{2} \operatorname{tr} \mathbf{R}\left[\mathbf{U D}(1,1,0) \mathbf{U}^{+}\right] \\
\mathbf{B}^{(2)} & \equiv \frac{1}{3} \operatorname{tr} \mathbf{R}\left[\mathbf{U D}(1,1,1) \mathbf{U}^{+}\right] .
\end{aligned}
$$

$\mathbf{A}^{(0)}$ is the pure component, $\mathbf{B}^{(1)}$ is the $2 \mathrm{D}$ unpolarized state and $\mathbf{B}^{(2)}$ is the $3 \mathrm{D}$ unpolarized state.

In later sections devoted to coherency matrices representing the polarimetric properties of material media, we 
will see that any physically realizable decomposition of such coherency matrices has the form of a linear convex combination of coherency matrices with equal traces.

The trivial decomposition constitutes an appropriate framework for distinguishing the pure component from the random or noise component of the radiation field. In the same sense it allows a proper treatment of the measurement errors when it is known that the polarization state being measured is pure.

\subsection{Entropy associated with $\mathbf{R}$}

From the description of the von Neumann entropy for $n$ dimensional density matrices introduced by Fano [46] and studied by Brosseau [2], the 3D polarization entropy can be defined as

$$
S_{(3)}=-\operatorname{tr}(\hat{\mathbf{R}} \ln \hat{\mathbf{R}})=-\sum_{i=0}^{2}\left(\hat{\lambda}_{i} \ln \hat{\lambda}_{i}\right)
$$

where $\hat{\mathbf{R}} \equiv \mathbf{R} / \operatorname{tr} \mathbf{R}$ is the density matrix and $\hat{\lambda}_{0}, \hat{\lambda}_{1}, \hat{\lambda}_{2}$ are the eigenvalues of $\hat{\mathbf{R}}$.

Now, in order to complete the study of the physical parameters involved in 3D polarized light, and in the light of the above results, we consider the entropy of a state of polarization in terms of the indices of purity of $\mathbf{R}$

$$
\begin{aligned}
S_{(3)}= & -\operatorname{tr}(\hat{\mathbf{R}} \ln \hat{\mathbf{R}})=-\sum_{i=0}^{2}\left(\hat{\lambda}_{i} \ln \hat{\lambda}_{i}\right) \\
= & -\left\{\frac{\left(1+\frac{1}{2} P_{2}+\frac{3}{2} P_{1}\right)}{3} \ln \left[\frac{1}{3}\left(1+\frac{1}{2} P_{2}+\frac{3}{2} P_{1}\right)\right]\right. \\
& +\frac{\left(1+\frac{1}{2} P_{2}-\frac{3}{2} P_{1}\right)}{3} \ln \left[\frac{1}{3}\left(1+\frac{1}{2} P_{2}-\frac{3}{2} P_{1}\right)\right] \\
& \left.+\frac{\left(1-P_{2}\right)}{3} \ln \left[\frac{1}{3}\left(1-P_{2}\right)\right]\right\}
\end{aligned}
$$

$S$ is characterized univocally by $P_{1}$ and $P_{2}$. The maximum $S_{(3)}=\ln 3$ corresponds to $P_{1}=P_{1}=P_{(3)}=0$, whereas the minimum $S_{(3)}=0$ is reached when $P_{1}=$ $P_{1}=P_{(3)}=1$.

Due to the relevant nature of the concepts related with entropy, Réfrégier et al. [108,114,115] have dealt with the Shannon entropy and with the Kullback relative entropy, including their relations with the $n$-dimensional degrees of polarization for optical waves with Gaussian and nonGaussian probability density functions. In these works, the degree of polarization is related to the measure of proximity between probability density functions and to the measure of disorder provided by the Shannon entropy.

Taking into account the expression of the von Neumann entropy associated with $2 \times 2$ coherency matrices given by equation (34), as well as that in the case of $3 \times 3$ coherency matrices there exist two indices of purity defined as relative differences of the eigenvalues, it is possible to introduce a proper definition of two respective "partial entropies", namely, directional entropy $S_{(2)}\left(P_{2}\right)$ and polarization entropy $S_{(2)}\left(P_{1}\right)$.
These invariant quantities contain objective information about the randomness in the propagation direction and in the polarization ellipse respectively. The condition $0 \leqslant P_{1} \leqslant P_{2} \leqslant 1$ on the indices of purity has its counterpart in the partial entropies $0 \leqslant S_{(2)}\left(P_{2}\right) \leqslant S_{(2)}\left(P_{1}\right) \leqslant$ $\ln 2$.

Moreover, specific partial entropy can be defined for each purity parameter $p_{i j}=\hat{\lambda}_{i}-\hat{\lambda}_{j}$. Nevertheless, as we have seen, $P_{1}, P_{2}$ contain all the relevant information and, hence, $S_{(2)}\left(P_{2}\right), S_{(2)}\left(P_{1}\right)$ are proper representative quantities among all possible partial entropies $S_{(2)}\left(p_{i j}\right)$.

Furthermore, following the definition of Réfrégier, Roche and Goudail [107] of the polarimetric contrast, we find that $\left[\operatorname{tr} \mathbf{R}, P_{1}, P_{2}\right]$ can be considered as an appropriate set of characteristic parameters of the polarimetric contrast of the corresponding $3 \times 3$ coherency matrix $\mathbf{R}$.

\section{Mathematical representation of the polarimetric effects of material media}

The polarimetric effects of a material sample depend on its intrinsic properties, on the spectral characteristics of interacting light, and on the particular type of interaction under study. Thus, given a direction of incidence of the light, it is possible to study the effects in the light refracted, reflected, diffracted or scattered in different directions. The transformation of the polarization state of the incoming light into the polarization state of the outgoing light due to the interaction with a passive linear medium can be represented through a linear transformation of the corresponding Stokes parameters [10-13].

The main problem of polarimetry is the physical interpretation of the information that provides the sixteen elements of the transformation matrix (Soleillet-Mueller matrix). The resolution of this problem requires a suitable mathematical characterization of these matrices, which is obtained through the construction of a $4 \times 4$ coherency matrix whose properties are analogous to that of the $2 \times 2$ and $3 \times 3$ coherency matrices studied previously. In order to avoid confusion, we will hereafter refer to the abovementioned transformation matrix as a Mueller matrix, rather than a Soleillet matrix, because this is the term generally used in the literature.

In the case of deterministic non-depolarizing linear material systems (pure systems), pure states are transformed into pure states, so that these basic interactions involve birefringence and diattenuation properties. In the general case, the incoherent superposition of emerging light pencils results in depolarization phenomena.

In this section, a general characterization of physical Mueller matrices is obtained from the representation of a passive, linear optical system as a parallel combination of pure elements. This model is justified because of the essentiality of scattering phenomena: any polarimetric behavior of a material medium is derived from the secondary radiation of the accelerated atomic and molecular charges of the medium.

This general characterization is obtained by means of a complete set of explicit necessary and sufficient conditions 


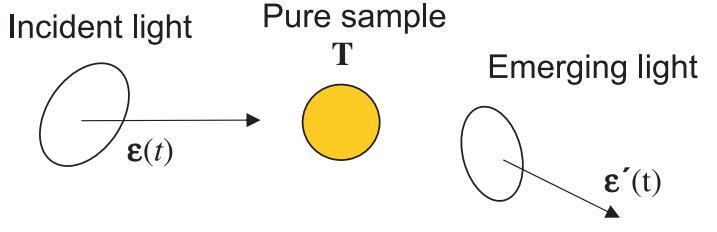

Fig. 6. (Color online) Basic polarimetric interaction.

for a real $4 \times 4$ matrix to be a physical Mueller matrix. A subset of four conditions is derived from the mathematical properties of the covariance matrix associated with a Mueller matrix (covariance conditions). Another subset of conditions, composed of two "transmittance conditions" is based on the physical restriction that the elements of the parallel combination do not amplify the intensity of light.

\subsection{Basic polarimetric interaction: the Jones matrix}

First, let us consider the Jones formalism in order to represent the effects of a deterministic-nondepolarizing optical system (or, briefly, "pure system") on the polarization properties of an electromagnetic wave that interacts with it. It should be noted that we use the term "deterministic nondepolarizing" in the sense that totally polarized incident light always emerges totally polarized. This observation is important because, as Simon has pointed out [116], the degree of polarization of a partially polarized light beam can decrease when it interacts with some kinds of deterministic optical systems (for example partial polarizers). Moreover, there exists the possibility of depolarizing effects when polychromatic light passes through some deterministic systems [117-120].

For passive pure systems, the linear transformation of the electric field components of the light beam interacting with them is given by the corresponding Jones matrix $\mathbf{T}$

$$
\varepsilon^{\prime}(t)=\mathbf{T} \varepsilon(t),
$$

where $\varepsilon^{\prime}, \varepsilon$, are the emerging and incident Jones vectors (Fig. 6).

Thus, we can say that any polarimetrically-pure system can be represented by its corresponding Jones matrix and vice-versa.

In general both emerging and incident fields can fluctuate, so that, in order to describe the properties of partially polarized light, it is necessary to use the $2 \times 2$ coherency matrices rather than the Jones vectors. The transformation of the coherency matrix is given by

$$
\begin{aligned}
\boldsymbol{\Phi}^{\prime} & =\left\langle\varepsilon^{\prime} \otimes \varepsilon^{\prime+}\right\rangle=\left\langle\mathbf{T} \boldsymbol{\varepsilon} \otimes(\mathbf{T} \varepsilon)^{+}\right\rangle \\
& =\left\langle\mathbf{T} \boldsymbol{\varepsilon} \otimes \varepsilon^{+} \mathbf{T}^{+}\right\rangle=\mathbf{T}\left\langle\varepsilon \otimes \varepsilon^{+}\right\rangle \mathbf{T}^{+}=\mathbf{T} \mathbf{\Phi} \mathbf{T}^{+} .
\end{aligned}
$$

Like other authors $[13,121]$, and in order to make subsequent straightforward calculations, we introduce the following alternative notation for the elements of $\boldsymbol{\Phi}$

$$
\varphi_{0} \equiv \varphi_{11}, \varphi_{1} \equiv \varphi_{12}, \varphi_{2} \equiv \varphi_{21}, \varphi_{3} \equiv \varphi_{22} .
$$

The elements of the polarization matrix can also be written as a column-vector $\varphi$ defined as

$$
\begin{aligned}
\boldsymbol{\varphi} & \equiv\left(\varphi_{0}, \varphi_{1}, \varphi_{2}, \varphi_{3}\right)^{T} \\
& =\left(\left\langle\varepsilon_{1} \varepsilon_{1}^{*}\right\rangle,\left\langle\varepsilon_{1} \varepsilon_{2}^{*}\right\rangle,\left\langle\varepsilon_{2} \varepsilon_{1}^{*}\right\rangle,\left\langle\varepsilon_{2} \varepsilon_{2}^{*}\right\rangle\right)^{T}=\left\langle\varepsilon \otimes \varepsilon^{*}\right\rangle .
\end{aligned}
$$

The polarization vector $\varphi$ and the corresponding Stokes vector $\mathbf{s}$ are related by means of the following expression $[13,47]$

$$
\mathbf{s}=\mathbf{L} \varphi
$$

where

$$
\mathbf{L}=\left[\begin{array}{cccc}
1 & 0 & 0 & 1 \\
1 & 0 & 0 & -1 \\
0 & 1 & 1 & 0 \\
0 & i & -i & 0
\end{array}\right]
$$

This matrix $\mathbf{L}$ connecting both vectorial representations of the polarization states has the following property

$$
\mathbf{L}^{-1}=\frac{1}{2} \mathbf{L}^{+} \text {. }
$$

Taking into account some properties of the Kronecker product, it is straightforward to show that the Stokes vector $\mathbf{s}^{\prime}$ of the emerging beam is given by

$$
\begin{aligned}
& \mathbf{s}^{\prime}=\mathbf{L}\left\langle\varepsilon^{\prime} \otimes \varepsilon^{* *}\right\rangle=\mathbf{L}\left\langle(\mathbf{T} \varepsilon) \otimes(\mathbf{T} \varepsilon)^{*}\right\rangle \\
&=\mathbf{L}\left\langle\left(\mathbf{T} \otimes \mathbf{T}^{*}\right)\left(\varepsilon \otimes \varepsilon^{*}\right)\right\rangle=\mathbf{L}\left(\mathbf{T} \otimes \mathbf{T}^{*}\right)\left\langle\left(\varepsilon \otimes \varepsilon^{*}\right)\right\rangle \\
&=\mathbf{L}\left(\mathbf{T} \otimes \mathbf{T}^{*}\right) \boldsymbol{\varphi}=\mathbf{L}\left(\mathbf{T} \otimes \mathbf{T}^{*}\right) \mathbf{L}^{-1} \mathbf{s} .
\end{aligned}
$$

Therefore, these linear transformations of Stokes vectors are given by the "Mueller-Jones matrix" N [13]

$$
\mathbf{N}=\mathbf{L}\left(\mathbf{T} \otimes \mathbf{T}^{+}\right) \mathbf{L}^{-1}
$$

or, in components,

$$
n_{k l}=\frac{1}{2} \operatorname{tr}\left(\boldsymbol{\sigma}_{k} \mathbf{T} \boldsymbol{\sigma}_{l} \mathbf{T}^{+}\right)
$$

In the next section we will deal with optical systems whose effect on polarized light cannot be represented by means of Jones matrices but can be represented by Mueller matrices. Therefore, like other authors, we will distinguish between Mueller-Jones matrices (or pure Mueller matrices [122]), which correspond to pure systems (i.e. characterized by Jones matrices) and Mueller matrices in general.

For the sake of clarity, hereafter we will use the following notation for the different types of matrices: $\mathbf{N}$, Mueller-Jones matrices and M, general Mueller matrices.

As several authors have pointed out $[17,18,45,123-126]$, the condition for the elements of the Jones matrix to represent a pure "physically realizable" (or physical) system arises from the physical restriction that the gain (intensity transmittance) $g$ of the optical system, defined as the ratio between the intensities of the emerging and incident light beams, must be limited to the interval $0 \leqslant g \leqslant 1$. This condition, 


$$
\begin{aligned}
& \mathbf{T}_{R}(\alpha, \delta, \Delta) \equiv\left(\begin{array}{cc}
c_{\alpha}^{2} e^{i(\Delta / 2)}+s_{\alpha}^{2} e^{-i(\Delta / 2)} & s_{\alpha} c_{\alpha}\left(e^{i(\Delta / 2)}-e^{-i(\Delta / 2)}\right) e^{-i \delta} \\
s_{\alpha} c_{\alpha}\left(e^{i(\Delta / 2)}-e^{-i(\Delta / 2)}\right) e^{i \delta} & s_{\alpha}^{2} e^{i(\Delta / 2)}+c_{\alpha}^{2} e^{-i(\Delta / 2)}
\end{array}\right), \\
& s_{\alpha} \equiv \sin \alpha, c_{\alpha} \equiv \cos \alpha
\end{aligned}
$$

called the "transmittance condition" (or "gain condition") can be written in function of the elements of $\mathbf{T}$ as follows $[124,126]$

$$
\frac{1}{2}\left\{\operatorname{tr}\left(\mathbf{T}^{+} \mathbf{T}\right)+\left[\left(\operatorname{tr}\left(\mathbf{T}^{+} \mathbf{T}\right)\right)^{2}+4 \operatorname{det}\left(\mathbf{T}^{+} \mathbf{T}\right)\right]^{1 / 2}\right\} \leqslant 1 .
$$

It should be noted that the condition $0 \leqslant g$, is directly satisfied [124].

Moreover, it is also opportune to consider the reversibility properties of the matrices that represent optical systems, i.e. the operation of interchanging the incident and emerging light beams. It was Jones who first formulated a reciprocity theorem in terms of Jones matrices [9]. Given a linear optical system characterized by a Jones matrix $\mathbf{T}$, the relation between the input $\boldsymbol{\eta}$ and output $\boldsymbol{\eta}^{\prime}$ Jones vectors when the light passes through the same system in the opposite direction is given by $\boldsymbol{\eta}^{T}=\boldsymbol{\eta}^{T} \boldsymbol{T}$, which formulated in terms of column vectors is

$$
\boldsymbol{\eta}^{\prime}=\mathbf{T}^{T} \boldsymbol{\eta}
$$

That is to say, the Jones matrix representing the system when incident and emerging beams are interchanged is $\mathbf{T}^{T}[9,127-129]$. This result is not applicable when the system exhibits magneto-optic effects. For example, for a Faraday cell, the forward-propagating and counterpropagating Jones matrices are equal [129].

Moreover, the symmetry of equation (107) shows that if it is valid for $\mathbf{T}$, it is also valid for $\mathbf{T}^{T}$. Therefore, if a Jones matrix $\mathbf{T}$ is physically realizable, $\mathbf{T}^{T}$ is also physically realizable, and vice-versa.

For a Mueller-Jones matrix $\mathbf{N}(\mathbf{T})$, the corresponding "reciprocal Mueller-Jones matrix" $\mathbf{N}^{r}$ is given by

$$
\mathbf{N}^{r} \equiv \mathbf{N}\left(\mathbf{T}^{T}\right)=\mathbf{D}(1,1,1,-1) \mathbf{N}^{T} \mathbf{D}(1,1,1,-1),
$$

where the diagonal matrix $\mathbf{D}(1,1,1,-1)$ performs a change of the sign in $s_{3}$ in the reverse interaction. As in the case of Jones matrices, a particular analysis of the form of $\mathbf{N}^{r}$ is required when the system includes magneto-optic effects.

The transmittance condition, expressed in function of the elements of $\mathbf{N}(\mathbf{T})$, is the following [124]

$$
g_{f} \leqslant 1, \quad g_{f} \equiv n_{00}+\left(n_{01}^{2}+n_{02}^{2}+n_{03}^{2}\right)^{1 / 2},
$$

where $g_{f}$ is the maximum transmittance of $\mathbf{N}$.

In the case of Mueller-Jones matrices, the following equality is satisfied $[130,131]$

$$
\left(n_{01}^{2}+n_{02}^{2}+n_{03}^{2}\right)=\left(n_{10}^{2}+n_{20}^{2}+n_{30}^{2}\right),
$$

so that the transmittance condition can also be expressed as

$$
g_{r} \leqslant 1, \quad g_{r} \equiv n_{00}+\left(n_{10}^{2}+n_{20}^{2}+n_{30}^{2}\right)^{1 / 2},
$$

which shows that

$$
g_{r}(\mathbf{N})=g_{f}\left(\mathbf{N}^{T}\right) .
$$

In consequence, $g_{r}$ can be properly considered as the maximum reverse transmittance. We will see that, although for Mueller-Jones matrices both "gains" are equal $g_{r}(\mathbf{N})=g_{f}(\mathbf{N})$, this equality fails, in general, for Mueller matrices: $g_{r}(\mathbf{M}) \neq g_{f}(\mathbf{M})$.

The above conclusions are also valid when the medium exhibits magneto-optic effects because changes of the sign in $m_{0 i}, m_{j 0}(i, j=1,2,3)$ do not affect the value of the maximum gain.

In a later section we will show that any system is polarimetrically equivalent to a certain parallel combination of pure systems, and that any pure system is polarimetrically equivalent to a serial combination of pure retarders and pure diattenuators (partial or total polarizers) [132]. The following sections are devoted to the properties of pure systems, prior to dealing with non-pure systems.

\subsection{Pure retarders}

Pure retarders are non-absorbing materials that exhibit different refraction indices (birefringence) for respective orthogonal states of polarization, and are represented by unitary Jones matrices

$$
\text { see equation (114) above }
$$

where $\Delta$ stands for the retardance caused between the two orthogonal elliptical-polarized eigenstates (Fig. 7). Their respective azimuths, (namely $\varphi, \varphi+\pi / 2$ ) and ellipticities $(\chi,-\chi)$ are given by

$\tan 2 \varphi=\tan 2 \alpha \cos \delta, \sin 2 \chi=\sin 2 \alpha \sin \delta$,

$$
(0 \leqslant \alpha \leqslant \pi / 2,-\pi<\delta \leqslant \pi) .
$$

The operational notation used for the Jones matrices is similar to that used by Priebe [133] for Mueller-Jones matrices.

It is important to point out that birefringent systems can produce depolarization in quasimonochromatic light. For instance, this is the case with long fiber optics, where the shift between the modes is higher than the coherence length. Thus, as we have indicated previously, these cases are not covered by the Jones model, which can only be applied when the cumulated shift is smaller than the coherence length of the light. The treatment of linear interactions involving depolarizing effects requires the use of the Mueller formalism.

Moreover, the chromatic dependence of the effective retardance of retarders is applied to the design of 


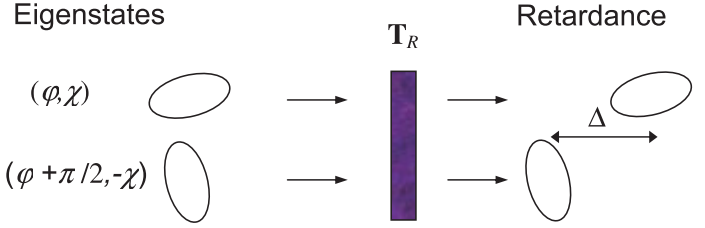

Fig. 7. (Color online) Pure retardes.

Lyot and Solc filters, which are constituted by appropriate serial combinations of retarders placed between total polarizers. The spectral calculations can be performed through the Jones formalism, whereas the study of the integrated depolarization effects requires the Mueller formalism $[117,134]$. All these considerations also have important applications to liquid crystal optical filters, electro-optical polarization controllers, polarization maintaining fiber ring lasers, birefringent tuning in dye lasers, thin film filters for optical sensing of gas concentration, active filters for color imaging, optical flip-flop systems and many other industrial and scientific devices.

Particular cases of pure retarders are the linear retarder $\mathbf{T}_{R}(\alpha, 0, \Delta)$ and the circular retarder $\mathbf{T}_{R}(\pi / 4, \pi / 2, \Delta)$.

Given a Jones matrix $\mathbf{T}$ referred to a coordinate system $X Y$ ( $Z$ is the propagation direction of the light beam), the corresponding Jones matrix $\mathbf{T}^{\prime}$ referred to a rotated coordinate system $X^{\prime} Y^{\prime}\left(\theta\right.$ : angle between $X$ and $\left.X^{\prime}\right)$ is given by

$$
\begin{aligned}
\mathbf{T}_{R}^{\prime} & \equiv \mathbf{T}_{G}(-\theta) \mathbf{T}_{R} \mathbf{T}_{G}(\theta) ; \\
\mathbf{T}_{G}(\theta) & \equiv\left(\begin{array}{cc}
\cos \theta & \sin \theta \\
-\sin \theta & \cos \theta
\end{array}\right) .
\end{aligned}
$$

With this operational notation for the unitary Jones matrices it is easy to show the following equivalences $[127,130,132,135-137]$ :

(a) $\mathbf{T}_{R}(\alpha, 0, \Delta)=\mathbf{T}_{G}(-\alpha) \mathbf{T}_{R}(0,0, \Delta) \mathbf{T}_{G}(\alpha)$,

(b) $\mathbf{T}_{R}^{-1}(\alpha, \delta, \Delta)=\mathbf{T}_{R}^{+}(\alpha, \delta, \Delta)=\mathbf{T}_{R}(\alpha, \delta,-\Delta)$,

(c) $\mathbf{T}_{R}(0,0,0)=\mathbf{D}(1,1)$,

(d) $\mathbf{T}_{R}(0,0, \pi)=\mathbf{D}(1,-1)$,

(e) $\mathbf{T}_{R}(0, \pi / 2, \Delta)=\mathbf{T}_{R}(0,0,-\Delta)$,

(f) $\mathbf{T}_{G}(\theta)=\mathbf{T}_{R}(\pi / 4, \pi / 2,2 \theta)$ : A rotation matrix is equivalent to the matrix of a circular retarder with $\Delta=2 \theta$,

(g) a system composed of any serial combination of retarders is equivalent to an elliptical retarder,

(h) an elliptical retarder is equivalent to a serial combination of a linear retarder and a circular retarder (rotator),

(i) an elliptical retarder is equivalent to a serial combination of two linear retarders,

(j) $\quad \mathbf{T}_{R}(\alpha, \delta, \Delta)=\mathbf{T}_{R}(0,0,-\delta) \mathbf{T}_{R}(\alpha, 0, \Delta) \mathbf{T}_{R}(0,0, \delta)$. Any pure retarder is optically equivalent to a linear retarder placed between two linear retarders whose fast axes coincide with the reference axes $X$ and $Y$ respectively,

(k) $\quad \mathbf{T}_{R}(\alpha, 0, \Delta)=\mathbf{T}_{R}\left(0,0, \delta_{1}\right) \mathbf{T}_{R}\left(\theta, 0, \delta_{2}\right) \mathbf{T}_{R}\left(0,0, \delta_{1}\right)$ : a linear retarder placed between two "aligned" equal linear retarders is equivalent to a linear retarder [138]. The parameters $\alpha, \Delta$ of the resulting equivalent linear retarder are

$$
\begin{aligned}
\cos (\Delta / 2) & =\cos \delta_{1} \cos \left(\delta_{2} / 2\right)-\sin \delta_{1} \sin \left(\delta_{2} / 2\right) \cos 2 \theta \\
\tan 2 \alpha & =\frac{\sin 2 \theta}{\sin \delta_{1} \cot \left(\delta_{2} / 2\right)+\cos \delta_{1} \cos 2 \theta} .
\end{aligned}
$$

This serial combination of three linear retarders provides a simple method for designing tunable compensators by adjusting the angle of the intermediate retarder.

In agreement with theorem $(j)$, any unitary Jones matrix $\mathbf{T}_{R}$ can be expressed as $\mathbf{T}_{R}=e^{i \mathbf{K}}$, where $\mathbf{K}$ is the Hermitian matrix given by $\mathbf{K}=$ $\left.\left.i[\mathbf{D}(1,1))-\mathbf{T}_{R}\right][\mathbf{D}(1,1))+\mathbf{T}_{R}\right]^{-1}[103]$. Thus, from this point of view, any pure retarder has an associated Hermitian matrix $\mathbf{K}$, in such a manner that its Jones matrix has the above exponential form. This representation is useful for calculations concerning the evolution of polarization states along the direction of propagation of the wave inside thick media [139-141]. In these problems, differential forms of Jones matrices and Mueller-Jones matrices are used $[139,142-144]$.

An interesting case of pure retarder is a twisted optical fiber with local linear polarized eigenstates. The twist results in an overall behavior equivalent to an elliptical retarder. Nevertheless, the appropriate physical model for the study of polarization mode dispersion is given by the "principal states of polarization" $[145,146]$. Despite the overall equivalence to an elliptical retarder, the system is also equivalent to a linear retarder followed by a circular retarder (rotator). Thus, the incoming eigenstates of the equivalent linear retarder result in a pair of orthogonal linear polarized outgoing states, which are rotated with respect to the incoming ones. As we will see, this model of principal states of polarization is also related with the singular value decomposition of Jones and Mueller matrices, and can be very useful for modeling serial and parallel combinations of pure systems.

The Jones formulation presented for pure retarders can easily be translated to the Mueller formalism. We have seen that any matrix of pure retarder can be expressed as a product of simple matrices corresponding to linear retarders and rotation matrices. The respective Mueller matrices of linear retarders and rotation matrices have the following forms

$$
\begin{aligned}
\mathbf{N}_{R}(0,0, \Delta) & =\left(\begin{array}{llll}
1 & 0 & 0 & 0 \\
0 & 1 & 0 & 0 \\
0 & 0 & c_{\Delta} & s_{\Delta} \\
0 & 0 & -s_{\Delta} & c_{\Delta}
\end{array}\right), \\
\mathbf{N}_{G}(\theta) & =\mathbf{N}_{R}(\pi / 4, \pi / 2,2 \theta)=\left(\begin{array}{lllll}
1 & 0 & 0 & 0 \\
0 & c_{2 \theta} & s_{2 \theta} & 0 \\
0 & -s_{2 \theta} & c_{2 \theta} & 0 \\
0 & 0 & 0 & 1
\end{array}\right)
\end{aligned}
$$


so that, in the same way as in the Jones model,

$$
\begin{aligned}
& \mathbf{N}_{R}(\alpha, \delta, \Delta)=\mathbf{N}_{R}(0,0,-\delta) \mathbf{N}_{R}(\alpha, 0, \Delta) \mathbf{N}_{R}(0,0, \delta) \\
& =\mathbf{N}_{R}(0,0,-\delta) \mathbf{N}_{G}(-\alpha) \mathbf{N}_{R}(0,0, \Delta) \mathbf{N}_{G}(\alpha) \mathbf{N}_{R}(0,0, \delta) .
\end{aligned}
$$

These orthogonal matrices have the general form

$$
\mathbf{N}_{R}=\left(\begin{array}{llll}
1 & 0 & 0 & 0 \\
0 & & \\
0 & \left(\overline{\mathbf{N}}_{R}\right) & \\
0 & &
\end{array}\right),
$$

where $\overline{\mathbf{N}}_{R}$ represents a proper rotation around the axes defined by the three Stokes parameters $s_{1}, s_{2}, s_{2}$, i.e. it corresponds to a rotation in the Poincaré sphere. It should be noted that, in accordance with the definition of the Stokes parameters, these axes do not correspond to those of the spatial reference system $X Y Z$.

Orthogonal Mueller matrices (which always correspond to pure retarders) can be diagonalized through the following similarity transformation

$$
\begin{aligned}
& \mathbf{D}\left(1,1, e^{i \Delta}, e^{-i \Delta}\right)=\mathbf{N}_{R}(0,0, \delta) \mathbf{N}_{G}(\alpha) \\
& \quad \times \mathbf{C}^{+} \mathbf{N}_{R}(\alpha, \delta, \Delta) \mathbf{C N}_{G}(-\alpha) \mathbf{N}_{R}(0,0,-\delta),
\end{aligned}
$$

where the non-Mueller unitary matrix $\mathbf{C}$ is [147]

$$
\mathbf{C}=\frac{1}{\sqrt{2}}\left(\begin{array}{cccc}
1 & 1 & 0 & 0 \\
1 & -1 & 0 & 0 \\
0 & 0 & 1 & 1 \\
0 & 0 & i & -i
\end{array}\right)
$$

Although this "modal matrix" is not the only one that satisfies equation (120), it has been chosen because it is the same as we will use to diagonalize the Mueller matrices of diattenuators.

Thus, any pure retarder has two physical eigenstates, with eigenvalues equal to one, which are the following [148]

$$
\begin{aligned}
\mathbf{t}^{(0)} & =\frac{1}{\sqrt{2}}\left(1, c_{2 \alpha}, s_{2 \alpha} c_{\delta}, s_{2 \alpha} s_{\delta}\right)^{T}, \\
\mathbf{t}^{(1)} & =\frac{1}{\sqrt{2}}\left(1,-c_{2 \alpha},-s_{2 \alpha} c_{\delta},-s_{2 \alpha} s_{\delta}\right)^{T},
\end{aligned}
$$

and the Mueller matrix also has the following two non-physical eigenvectors (with respective eigenvalues $\left.e^{i \Delta}, e^{-i \Delta}\right)$

$$
\begin{aligned}
& \frac{1}{\sqrt{2}}\left(1,-s_{2 \alpha}, c_{2 \alpha} c_{\delta}+i s_{\delta}, c_{2 \alpha} s_{\delta}-i c_{\delta}\right)^{T} \\
& \frac{1}{\sqrt{2}}\left(1,-s_{2 \alpha}, c_{2 \alpha} c_{\delta}-i s_{\delta}, c_{2 \alpha} s_{\delta}+i c_{\delta}\right)^{T} .
\end{aligned}
$$

The action of a retarder can be represented in the Poincaré sphere as a rotation, of an angle $\Delta$, around the axis defined by the two antipodal points corresponding to the two orthogonal physical eigenstates [127].

The matrix $\overline{\mathbf{N}}_{R}$ characteristic of a pure retarder can be expressed in terms of the normalized Stokes eigenvector for the fast axis $\hat{\mathbf{t}}^{(0)} \equiv \mathbf{t}^{(0)} / t_{0}^{(0)}$ and the retardance as follows [149]

$$
\begin{aligned}
\left(\overline{\mathbf{N}}_{R}\right)_{i j}=\delta_{i j} & \cos \Delta+\hat{t}_{i}^{(0)} \hat{t}_{j}^{(0)}(1-\cos \Delta) \\
& +\sum_{k=1}^{3}\left(\epsilon_{i j k} \hat{t}_{k}^{(0)}\right) \sin \Delta ; \quad i, j=1,2,3
\end{aligned}
$$

where $\delta_{i j}$ is the Kronecker delta and is the Lecy-Cività permutation symbol.

Conversely, the retardance and the elements of the eigenvectors of $\mathbf{N}_{R}$ can be obtained as [16,149]

$$
\cos \Delta=\frac{\operatorname{tr} \mathbf{N}_{R}}{2}-1, \quad \hat{t}_{i}^{(0)}=\frac{1}{2 \sin \Delta} \sum_{j, k=1}^{3} \epsilon_{i j k}\left(\overline{\mathbf{N}}_{R}\right)_{j k}
$$

It should also be noted that the transmittance of a pure retarder is independent of the incoming Stokes vector and $g\left(\mathbf{N}_{R}\right)=1$.

Any serial combination of pure retarders is equivalent to a certain elliptical retarder represented by a unitary Jones matrix (orthogonal Mueller matrix). Therefore, any kind of pure retarder has two orthogonal eigenstates.

Hereafter we will distinguish between serial combinations, where the light passes through successive elements arranged along the direction of propagation, and parallel combinations, where the incoming light beam falls simultaneously on different parts of the material target and the light pencils emerging from the different components are recombined into a whole emerging beam. The Mueller (or Jones) matrix of a serial combination is given by the ordered product of the Mueller (or Jones) matrices corresponding to the different components. The Jones matrix of a coherent parallel combination is given by a convex linear combination of the Jones matrices corresponding to the different components [13]. The Mueller matrix of an incoherent parallel combination is given by a convex linear combination of the Mueller matrices corresponding to the different components [13]. For a proper calculus of such a convex linear combination, the matrices of the components must be normalized to have the same mean transmittance as that exhibited by the whole equivalent matrix.

An original method for the graphical representation of retarders has been introduced on the basis of a fourdimensional spherical parameterization of the Jones matrix. This representation takes the form of a solid cylinder in such a manner that the projection of the point representing the retarder on the cylinder base gives the corresponding Jones eigenvectors [150].

Equation (119) shows the possibility of a particular treatment of Mueller-Jones matrices representing devices composed of retarders by means of $3 \times 3$ matrices applicable directly to the "vectorial part" of the Stokes vectors $\left(s_{1}, s_{2}, s_{3}\right)^{T}[137]$. Moreover, the use of a complex number to represent the polarization ellipse of light passing through a thick retarder allows us to obtain the evolution 
Eigenstates

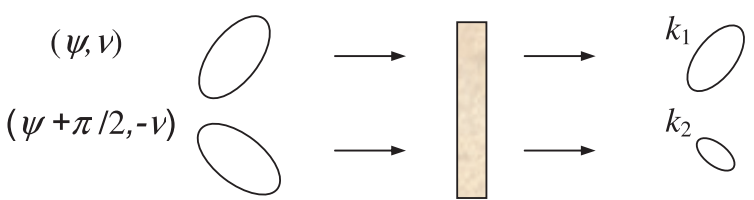

Fig. 8. (Color online) Pure diattenuator.

of the state of polarization along the direction of propagation. This evolution is given by a second-degree ordinary differential equation [151].

As we will see in next section, this $3 \times 3$ model cannot be applied to pure systems exhibiting diattenuation.

\subsection{Pure diattenuators}

Pure diattenuators are material systems exhibiting selective transmittances for two incoming states of polarization. A diattenuator is called "homogeneous" when it has two orthogonal eigenstates and is represented by a positive semidefinite Hermitian Jones matrix

$\mathbf{T}_{D}\left(\beta, \gamma, k_{1}, k_{2}\right) \equiv\left(\begin{array}{lc}k_{1} c_{\beta}^{2}+k_{2} s_{\beta}^{2} & s_{\beta} c_{\beta}\left(k_{1}-k_{2}\right) e^{-i \gamma} \\ s_{\beta} c_{\beta}\left(k_{1}-k_{2}\right) e^{i \gamma} & k_{1} s_{\beta}^{2}+k_{2} c_{\beta}^{2}\end{array}\right)$,

$s_{\beta} \equiv \sin \beta, \quad c_{\beta} \equiv \cos \beta$,

where $k_{1}, k_{2}\left(0 \leqslant k_{2} \leqslant k_{1} \leqslant 1\right)$ are the coefficients of amplitude transmittance for the two orthogonal eigenstates (Fig. 8). Their respective azimuths, (namely $\psi, \psi+\pi / 2$ ) and ellipticities $(\nu,-\nu)$ are given by

$$
\begin{array}{r}
\tan 2 \psi=\tan 2 \beta \cos \gamma, \quad \sin 2 \nu=\sin 2 \beta \sin \gamma, \\
(0 \leqslant \beta \leqslant \pi / 2,-\pi<\gamma \leqslant \pi) .
\end{array}
$$

Some interesting specific cases are the following: total diattenuator $\mathbf{T}_{D}\left(\beta, \gamma, k_{1}, 0\right)$; linear diattenuator $\mathbf{T}_{D}\left(\beta, 0, k_{1}, k_{2}\right)$, and circular diattenuator $\mathbf{T}_{D}\left(\pi / 4, \pi / 2, k_{1}, k_{2}\right)$.

The inverse matrix of a non-singular Hermitian Jones matrix is

$\mathbf{T}_{D}^{-1}\left(\beta, \gamma, k_{1}, k_{2}\right)=\mathbf{T}_{D}\left(\beta, \gamma, 1 / k_{1}, 1 / k_{2}\right)$

$$
\left(0<k_{2} \leqslant k_{1} \leqslant 1\right) \text {. }
$$

We observe that this is a non-Jones matrix because it violates the transmittance condition. For singular Hermitian Jones matrices $\left(k_{2}=0\right)$, the corresponding pseudoinverse is

$$
\mathbf{T}_{D}^{-}\left(\beta, \gamma, k_{1}, 0\right)=\mathbf{T}_{D}\left(\beta, \gamma, 1 / k_{1}, 0\right), \quad\left(0<k_{1} \leqslant 1\right),
$$

so that

$$
\left(\mathbf{T}_{D}^{-} \mathbf{T}_{D}\right) \mathbf{T}_{D}=\mathbf{T}_{D}
$$

When $k_{2}=k_{1}$ the diattenuator degenerates into a partially-transparent isotropic medium

$$
\mathbf{T}_{D}\left(\beta, \gamma, k_{1}, k_{1}\right)=k_{1} \mathbf{D}(1,1) .
$$

The matrix of a generic homogeneous elliptical diattenuator can be written as

$$
\begin{array}{r}
\mathbf{T}_{D}\left(\beta, \gamma, k_{1}, k_{2}\right)=\mathbf{T}_{R}(0,0,-\gamma) \mathbf{T}_{D}\left(\beta, 0, k_{1}, k_{2}\right) \\
\times \mathbf{T}_{R}(0,0, \gamma)=\mathbf{T}_{R}(0,0,-\gamma) \mathbf{T}_{G}(-\beta) \\
\times \mathbf{T}_{D}\left(0,0, k_{1}, k_{2}\right) \mathbf{T}_{G}(\beta) \mathbf{T}_{R}(0,0, \gamma)
\end{array}
$$

i.e. any elliptical diattenuator is optically equivalent to a linear diattenuator placed between two linear retarders whose fast axes coincide with the reference axes $X$ and $Y$ respectively.

It should be noted that the limits established for $k_{1}, k_{2}$, which are just the singular values of $\mathbf{T}_{D}$, ensure the fulfillment of the transmittance condition

$$
g\left(\mathbf{T}_{D}\right)=k_{1}^{2} \leqslant 1 .
$$

As in the case of pure retarders, the Mueller-Jones matrices allow the matricial equivalences shown for the Jones model to be replicated. Nevertheless, it is important to consider the Mueller-Jones matrices because they can be used in the general Stokes-Mueller model.

We have seen that any matrix of a pure homogeneous diattenuator can be expressed as a product of simple matrices corresponding to linear diattenuators, linear retarders (aligned with the reference axes) and rotation matrices. The Mueller matrix of an "aligned" $(\beta=0)$ linear diattenuator is

$$
\begin{aligned}
& \mathbf{N}_{D}\left(0,0, k_{1}, k_{1}\right) \\
& \quad=\frac{1}{2}\left(\begin{array}{cccc}
k_{1}^{2}+k_{2}^{2} & k_{1}^{2}-k_{2}^{2} & 0 & 0 \\
k_{1}^{2}-k_{2}^{2} & k_{1}^{2}+k_{2}^{2} & 0 & 0 \\
0 & 0 & 2 k_{1} k_{2} & 0 \\
0 & 0 & 0 & 2 k_{1} k_{2}
\end{array}\right),
\end{aligned}
$$

so that, as in the Jones model,

$$
\begin{array}{r}
\mathbf{N}_{D}\left(\beta, \gamma, k_{1}, k_{2}\right)=\mathbf{N}_{R}(0,0,-\gamma) \mathbf{N}_{D}\left(\beta, 0, k_{1}, k_{2}\right) \\
\times \mathbf{N}_{R}(0,0, \gamma)=\mathbf{N}_{R}(0,0,-\gamma) \mathbf{N}_{G}(-\beta) \\
\quad \times \mathbf{N}_{D}\left(0,0, k_{1}, k_{2}\right) \mathbf{N}_{G}(\beta) \mathbf{N}_{R}(0,0, \gamma)
\end{array}
$$

Mueller-Jones matrices corresponding to pure homogeneous diattenuators are symmetric and can be diagonalized through the following similarity transformation

$$
\begin{aligned}
\mathbf{D}\left(1,1, k_{1}^{2}, k_{2}^{2}\right)= & \mathbf{N}_{R}(0,0, \gamma) \mathbf{N}_{G}(\beta) \mathbf{C}^{+} \mathbf{N}_{D}\left(0,0, k_{1}, k_{2}\right) \\
& \times \mathbf{C N}_{G}(-\beta) \mathbf{N}_{R}(0,0,-\gamma) .
\end{aligned}
$$

Therefore, the eigenvalues of a pure diattenuator are $\left(k_{1}^{2}, k_{2}^{2}, k_{1} k_{2}, k_{1} k_{2}\right)$, which correspond to the same eigenvectors as those obtained for the Mueller-Jones matrices of pure retarders (but replacing $\alpha, \delta$ by $\beta, \gamma$ ) $[147,148]$. $k_{1}^{2}, k_{2}^{2}$ are the intensity transmittances of to the two physical eigenstates, whereas the duplicated eigenvalue $k_{1} k_{2}$ corresponds to the other two non-physical eigenvectors.

The Mueller matrix of a homogeneous diattenuator can be written as [149]

$$
\mathbf{N}_{D}=n_{00}\left[\begin{array}{ll}
1 & \mathbf{\Upsilon}^{T} \\
\mathbf{\Upsilon} & \overline{\mathbf{N}}_{D}
\end{array}\right]
$$


where

$$
\begin{aligned}
& \Upsilon \equiv \frac{\left(n_{10}, n_{20}, n_{30}\right)^{T}}{n_{00}}=\frac{\left(n_{01}, n_{02}, n_{03}\right)^{T}}{n_{00}}, \\
& \overline{\mathbf{N}}_{D}=\left(1-\Upsilon^{2}\right)^{1 / 2} \mathbf{D}(1,1,1)+\left[1-\left(1-\Upsilon^{2}\right)^{1 / 2}\right] \frac{\mathbf{\Upsilon} \otimes \boldsymbol{\Upsilon}^{\mathbf{T}}}{\Upsilon^{2}} ; \\
& \Upsilon \equiv|\Upsilon|
\end{aligned}
$$

These expressions show that the Mueller matrix is completely determined by its transmittance for unpolarized light $n_{00}$ and its "polarizance/diattenuation vector" $\mathbf{\Upsilon}$ [149], that is to say, $\mathbf{N}_{D}$ is completely characterized by the Stokes vector $n_{00}(1, \mathbf{\Upsilon})[152]$.

The parameters $\left(\beta, \gamma, k_{1}, k_{2}\right)$ of the homogeneous diattenuator can be calculated from $\mathbf{N}_{D}$ as [16]

$$
\begin{aligned}
\tan 2 \beta & =\frac{\left(n_{20}^{2}+n_{30}^{2}\right)^{1 / 2}}{n_{10}}=\frac{\left(n_{02}^{2}+n_{03}^{2}\right)^{1 / 2}}{n_{01}}, \\
\tan \gamma & =\frac{n_{30}}{n_{20}}=\frac{n_{03}}{n_{02}}, \\
k_{1}^{2} & =n_{00}(1+\Upsilon) ; \quad k_{2}^{2}=n_{00}(1-\Upsilon) .
\end{aligned}
$$

Any serial combination of pure diattenuators is equivalent to a diattenuator whose Jones matrix (Mueller matrix) is a product of Hermitian (symmetric) matrices. Unlike the case of unitary (orthogonal) matrices, the product of Hermitian (symmetric) matrices is not a Hermitian (symmetric) matrix. This means that the most general case of diattenuator has non-orthogonal eigenstates (inhomogeneous diattenuator). Moreover, general conditions for pure systems to be dichroic (diattenuator) and birefringent (retarder) have been studied by Savenkov, Sydoruk and Muttiah [153].

The effects of partial polarizers on the input field can also be represented in quantum theoretical terms by replacing the classical amplitude transmittances with their respective annihilation operators. This subject has been studied by Agarwal [154], showing that the Wigner function of the field is transformed in a simple manner by polarizing devices (squeezing devices) and finding the relation of the Berry phase with this kind of transformation.

\subsection{Polar decomposition of pure systems}

A general matricial description of pure systems is given by the polar decomposition of its corresponding matrices $[16,135]$. This decomposition can be carried out in both Jones and Mueller-Jones formalisms.

Any $2 \times 2$ complex matrix $\mathbf{T}$ that satisfies the transmittance condition given by equation (107) is a Jones matrix and can be written as the product of a Hermitian matrix (pure diattenuator) and a unitary matrix (pure retarder) in either of the two possible relative positions (Fig. 9):

$$
\begin{aligned}
& \mathbf{T}=\mathbf{T}_{D}\left(\beta, \gamma, k_{1}, k_{2}\right) \mathbf{T}_{R}(\alpha, \delta, \Delta) \\
& \mathbf{T}=\mathbf{T}_{R}\left(\alpha^{\prime}, \delta^{\prime}, \Delta^{\prime}\right) \mathbf{T}_{D}\left(\beta^{\prime}, \gamma^{\prime}, k_{1}^{\prime}, k_{2}^{\prime}\right) .
\end{aligned}
$$
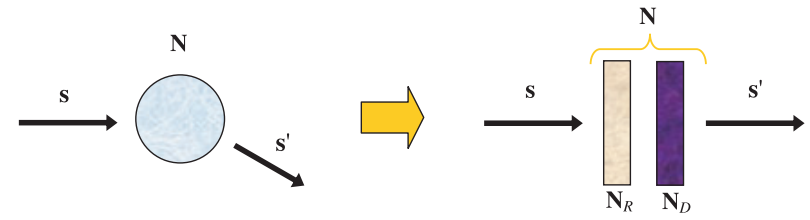

Fig. 9. (Color online) Retarder-diattenuator equivalent system based on the polar decomposition of pure matrices.

Obviously, these expressions are directly translatable to the corresponding Mueller-Jones matrices

$$
\begin{aligned}
& \mathbf{N}=\mathbf{N}_{D}\left(\beta, \gamma, k_{1}, k_{2}\right) \mathbf{N}_{R}(\alpha, \delta, \Delta) ; \\
& \mathbf{N}=\mathbf{N}_{R}\left(\alpha^{\prime}, \delta^{\prime}, \Delta^{\prime}\right) \mathbf{N}_{D}\left(\beta^{\prime}, \gamma^{\prime}, k_{1}^{\prime}, k_{2}^{\prime}\right),
\end{aligned}
$$

where $\mathbf{N}_{D}$ is a symmetric Mueller-Jones matrix and $\mathbf{N}_{R}$ is an orthogonal Mueller-Jones matrix.

The set of physical parameters $\left(\alpha, \delta, \Delta, \beta, \gamma, k_{1}, k_{2}\right)$, which are restricted to the limits

$$
\begin{aligned}
& 0 \leqslant \alpha \leqslant \pi / 2, \quad-\pi<\delta \leqslant \pi, \quad 0 \leqslant \Delta \leqslant \pi, \\
& \quad 0 \leqslant \beta \leqslant \pi / 2, \quad-\pi<\gamma \leqslant \pi, \quad 0 \leqslant k_{2} \leqslant k_{1} \leqslant 1,
\end{aligned}
$$

provides all the information obtainable through polarimetric techniques.

Alternatively, $\left(\alpha^{\prime}, \delta^{\prime}, \Delta^{\prime}, \beta^{\prime}, \gamma^{\prime}, k_{1}^{\prime}, k_{2}^{\prime}\right)$ can be used to characterize the pure system.

When $\mathbf{N}$ is singular $\left[\operatorname{det} \mathbf{N}=(\operatorname{det} \mathbf{T})^{4}=0\right]$ the polar decomposition is not unique and arbitrary values of $\delta, \delta^{\prime}$ can be chosen.

Regardless of whether $\mathbf{N}$ is singular or non-singular, the parameters corresponding to the equivalent diattenuators are [16]

$$
\begin{aligned}
k_{1}^{2} & =k_{1}^{\prime 2}=n_{00}(1+\Upsilon), \quad k_{2}^{2}=k_{2}^{\prime 2}=n_{00}(1-\Upsilon), \\
\tan 2 \beta & =\frac{\left(n_{20}^{2}+n_{30}^{2}\right)^{1 / 2}}{n_{10}}, \\
\tan 2 \beta^{\prime} & =\frac{\left(n_{02}^{2}+n_{03}^{2}\right)^{1 / 2}}{n_{01}}, \quad \tan \gamma=\tan \gamma^{\prime}=\frac{n_{30}}{n_{20}} \frac{n_{03}}{n_{02}},
\end{aligned}
$$

where

$\Upsilon \equiv \frac{1}{n_{00}}\left(n_{01}^{2}+n_{02}^{2}+n_{03}^{2}\right)^{1 / 2}=\frac{1}{n_{00}}\left(n_{10}^{2}+n_{20}^{2}+n_{30}^{2}\right)^{1 / 2}$.

This last equality expresses a particular property of pure Mueller matrices.

It should be noted that $k_{1}^{2}, k_{2}^{2}, k_{1} k_{2}, k_{1} k_{2}$ are the singular values of $\mathbf{N}(\mathbf{T})$ and that, obviously, the value of the maximum transmittance does not depend on the mathematical formalism used, i.e.

$$
\begin{aligned}
g(\mathbf{N}) & =g(\mathbf{T})=k_{1}^{2} \\
& =\frac{1}{2}\left\{\operatorname{tr}\left(\mathbf{T}^{+} \mathbf{T}\right)+\left[\left(\operatorname{tr}\left(\mathbf{T}^{+} \mathbf{T}\right)\right)^{2}+4 \operatorname{det}\left(\mathbf{T}^{+} \mathbf{T}\right)\right]^{1 / 2}\right\} \\
& \leqslant 1 .
\end{aligned}
$$


Moreover, the minimum transmittance $k_{2}^{2}$ can be expressed as

$$
k_{2}^{2}=\frac{1}{2}\left\{\operatorname{tr}\left(\mathbf{T}^{+} \mathbf{T}\right)-\left[\left(\operatorname{tr}\left(\mathbf{T}^{+} \mathbf{T}\right)\right)^{2}+4 \operatorname{det}\left(\mathbf{T}^{+} \mathbf{T}\right)\right]^{1 / 2}\right\}
$$

Now, for the obtainment of the parameters of the equivalent retarder, we distinguish two cases [16]:

(1) $\mathbf{N}$ non-singular $\left(0<k_{2}\right)$

As a first step, by applying the inverse matrices of the respective diattenuators, the matrices of the retarders are calculated

$$
\begin{aligned}
\mathbf{E} \equiv \mathbf{N}_{R}(\alpha, \delta, \Delta) & =\mathbf{N}_{D}\left(\beta, \gamma, 1 / k_{1}, 1 / k_{2}\right) \mathbf{N} \\
\mathbf{N}_{R}\left(\alpha^{\prime}, \delta^{\prime}, \Delta^{\prime}\right) & =\mathbf{N N}_{D}\left(\beta^{\prime}, \gamma^{\prime}, 1 / k_{1}, 1 / k_{2}\right)=\mathbf{E} .
\end{aligned}
$$

Furthermore, the angular parameters $\varphi, \chi, \Delta$ of the equivalent retarder are obtained through the following relations

$$
\begin{aligned}
\cos \Delta & =\cos \Delta^{\prime}=\frac{\operatorname{tr} \mathbf{E}}{2}-1, \\
\sin 2 \chi & =\sin 2 \chi=\frac{e_{12}-e_{21}}{2 \sin (\Delta / 2)} \\
\sin 2 \varphi & =\sin 2 \varphi^{\prime}=\frac{e_{31}-e_{13}}{2 \cos 2 \chi \sin (\Delta / 2)}
\end{aligned}
$$

which lead to

$$
\begin{aligned}
& \cos 2 \alpha=\cos 2 \alpha^{\prime}=\cos 2 \varphi \cos 2 \chi=\cos 2 \varphi^{\prime} \cos 2 \chi^{\prime} \\
& \tan \delta=\tan \delta^{\prime}=\frac{\tan 2 \chi}{\sin 2 \varphi}=\frac{\tan 2 \chi^{\prime}}{\sin 2 \varphi^{\prime}}
\end{aligned}
$$

(2) $\mathbf{N}$ singular $\left(0=k_{2}\right)$

In this case, the decomposition is not unique. An arbitrary value can be chosen for $\delta$, (or $\delta^{\prime}$ ) for example $\delta=0\left(\delta^{\prime}=0\right)$, so that the equivalent retarder is a linear retarder. Then $\alpha, \alpha^{\prime}$ are given by

$$
\tan 2 \alpha=\tan 2 \alpha^{\prime}=\frac{n_{10}-n_{01}}{n_{02}-n_{20}},
$$

and for $\Delta\left(\Delta^{\prime}\right)$, two different values $\Delta_{1}, \Delta_{2}\left(\Delta_{1}^{\prime}, \Delta_{2}^{\prime}\right)$ are possible

$$
\begin{aligned}
& \tan \Delta_{1}=\tan \Delta_{1}^{\prime}=\frac{b n_{30}-a n_{03}}{b^{2}-n_{03}^{2}} \\
& \tan \Delta_{2}=\tan \left(\pi-\Delta_{2}^{\prime}\right)=\frac{b n_{30}+a n_{03}}{b^{2}-n_{03}^{2}},
\end{aligned}
$$

where

$$
a \equiv n_{01} \sin \alpha-n_{02} \cos \alpha, \quad b \equiv n_{10} \sin \alpha-n_{20} \cos \alpha .
$$

In the case of $n_{03}=0$, these results must be replaced by $\Delta=\Delta^{\prime}=\pi / 2$.

Each one of the pure components of the equivalent system given by the polar decomposition is homogeneous [17], i.e. has orthogonal eigenstates. In particular, the fact that the equivalent diattenuator has orthogonal eigenstates (and, hence, has not the most general form of a pure diattenuator) does not represent a lack of validity, but is a peculiarity of the polar decomposition.

From the general expressions of the Jones matrices (or the corresponding Mueller-Jones matrices) of pure retarders, pure diattenuators and pure systems, several theorems can be stated. All of them can be deduced from those mentioned in this section $[127,130,132,135-137,155]$.

The equivalent model (retarder-diattenuator) of a pure system, which has been obtained by the polar decomposition of its corresponding matrix $\mathbf{T}$, results in the singular value decomposition of $\mathbf{T}$. In fact, equation (140) can be expressed as

$$
\begin{aligned}
\mathbf{T}=\left[\mathbf{T}_{R}(0,0,\right. & \left.-\gamma) \mathbf{T}_{G}(-\beta)\right] \mathbf{T}_{D}\left(0,0, k_{1}, k_{2}\right) \\
& \times\left[\mathbf{T}_{G}(\beta) \mathbf{T}_{R}(0,0, \gamma) \mathbf{T}_{R}(\alpha, \delta, \Delta)\right] .
\end{aligned}
$$

This Jones algebra allows us to easily distinguish between "homogeneous" Jones matrices (i.e. the eigenvalues coincide with the singular values) and "inhomogeneous" Jones matrices (i.e. the eigenvalues are different from the singular values). Homogeneous Jones matrices correspond to systems in which the eigenvectors of the equivalent retarder coincide with those of the equivalent diattenuator. A particular case of homogeneous Jones matrices are those that represent pure retarders. This subject has been studied by Lu and Chipman [17], who have defined an "inhomogeneity parameter" $\eta$ as the scalar product of the eigenvectors of the system, so that for homogeneous systems $\eta=0$, whereas the value $\eta=1$ represents the maximum possible value of the inhomogeneity. An example of system with $\eta=1$ is a serial combination composed of a retarder placed between two crossed total polarizers ("black sandwich") [156].

Obviously, given the direct relation between Jones and Mueller-Jones matrices, the above arguments are also valid for Muller-Jones matrices.

The above-mentioned authors, Lu and Chipman, have analyzed the retardation and diattenuation properties for both homogeneous and inhomogeneous cases through the polar decomposition of their Jones matrices [17,149]. In terms of the elements of the Mueller-Jones matrix $\mathbf{N}$, the polarizance and diattenuation vectors are defined as

$$
\Upsilon_{f} \equiv \frac{1}{n_{00}}\left(n_{10}, n_{20}, n_{30}\right)^{T}, \quad \Upsilon_{r} \equiv \frac{1}{n_{00}}\left(n_{01}, n_{02}, n_{03}\right)^{T},
$$

so that $\mathbf{\Upsilon}_{f}$ contain all the information about the polarizing power for incident unpolarized radiation, whereas $\mathbf{\Upsilon}_{r}$ describes completely the transmittance properties of $\mathbf{N}$.

Taking into account the reciprocity conditions for Mueller matrices, we see that

$$
\boldsymbol{\Upsilon}_{f}(\mathbf{N})=\Upsilon_{r}\left(\mathbf{N}^{r}\right), \quad \Upsilon_{r}(\mathbf{N})=\Upsilon_{f}\left(\mathbf{N}^{r}\right),
$$

and, from equation (144), we observe that, for pure systems, the modulus of both polarizance and diattenuation vectors are equal. 
The properties of non-singular Jones and MuellerJones matrices can be studied by means of their representation in the $\mathrm{SL}(2 \mathrm{C})$ group or in the proper orthochronous Lorentz group respectively [157-160]. In the case of diattenuators, this requires a normalization which violates the transmittance condition. As we will see, a powerful group-theoretic framework for the representations of the polarimetric properties of material media arises from the concept of the coherency matrix associated with a Mueller matrix $[41,161-163]$.

\section{Interaction of polarized light with non-deterministic passive optical systems}

Many scientific and industrial applications of polarimetry involve depolarization phenomena. The study and characterization of the general polarimetric behavior of material samples is of considerable importance in order to take maximum advantage of the potential possibilities of these non-destructive techniques.

In general, for an incident totally polarized light beam, a material system produces a superposition of totally polarized outgoing light pencils with different polarizations. Depending on the spectral distribution of the incident light, on the nature of the material target and on the nature of the interaction, these pencils can present different relative degrees of coherence. Thus, the superposition of some pencils can be coherent (so that their respective Jones vectors must be added in order to obtain the corresponding resultant Jones vector), the superposition of some pencils can be incoherent (so that their respective Stokes vectors must be added in order to obtain the corresponding resultant Stokes vector) and the superposition of other outgoing pencils can be partially coherent (so that their coherent parts are "Jones-added" and their incoherent parts are "Stokes-added"). Taking into account the principle of optical equivalence of polarization states [33], the above observation implies that the polarimetric effect of a material medium is equivalent to that of a system composed of a parallel combination of several pure optical systems. Therefore, the state of polarization of the outgoing light beam is physically equivalent to the state of polarization of the superposition of the light beams emerging from each one of the pure components of the equivalent parallel combination.

In this paper, we deal only with physical (or physically realizable) Mueller matrices, i.e. $4 \times 4$ real matrices that represent the linear polarimetric behavior of material systems. In accordance with the above considerations, we will show that the Mueller matrix of the whole material system is given by a convex sum of the Mueller-Jones matrices of the incoherent pure elements included in the parallel combination. This definition of general Mueller matrices is equivalent to saying that the Mueller matrix of the system can be considered as an ensemble average of pure Mueller matrices [161,164-168].

Although in the literature the properties of matrices transforming Stokes vectors into Stokes vectors (i.e. sat- isfying the so-called "Stokes criterion") have been studied $[152,160,167,169-175]$, this kind of matrix, hereafter named "Stokes matrices", does not coincide with the set of "Mueller matrices", which is derived from the "ensemble criterion". Some properties of Stokes matrices have been obtained from the Stokes criterion, which means that the degree of polarization of outgoing light must be less than, or equal to, unity (i.e. the Stokes matrix does not "overpolarize") $[169,170,173]$. These and other relevant results have been derived from the properties of the eigenvectors of $\mathbf{G} \mathbf{M}^{T} \mathbf{G M}$, or $\mathbf{M}^{T} \mathbf{G M}$ and from their spectral decomposition, where $\mathbf{G}$ is the diagonal matrix $\mathbf{G} \equiv \mathbf{D}(1,-1,-1,-1)[160,172,174]$.

Obviously, any physical Mueller matrix is a Stokes matrix but, in general, the converse is not true. No method has been quoted to physically realize a Stokes matrix being non derivable from the ensemble criterion. It is obvious that only physical Mueller matrices (from now on, Mueller matrices) are the subject of physical interest and, as several authors have pointed out $[165,167,168]$, the ensemblebased model represents a suitable theoretical framework for the study of polarimetric phenomena. In the following sections we formulate mathematically the conditions for a $4 \times 4$ real matrix to be a Mueller matrix.

\subsection{Construction of a Mueller matrix}

In general, an optical system can exhibit spatial heterogeneity over the area illuminated by the incident light beam, as well as dispersive effects, producing depolarization. The emerging light is consequently composed of several incoherent contributions, and the optical system cannot be represented by means of a Jones matrix. However, taking into account that, essentially, any linear effect is due to certain sort of scattering, the system can be considered as being composed of deterministic-nondepolarizing elements, each one with a well-defined Jones matrix, in such a manner that the light beam is shared among these different elements. In other words, the system is a parallel combination of polarimetrically-pure components (Fig. 10).

Let $I^{(i)}$ be the intensity of the portion of light that interacts with the " $i$ " element. The ratio between $I^{(i)}$ and the intensity $I$ of the whole beam is denoted by a respective coefficient $p_{i}$ so that

$$
p_{i}=\frac{I^{(i)}}{I}, \quad \sum_{i} p_{i}=1
$$

Now we denote by $\mathbf{T}^{(i)}, \mathbf{N}^{(i)}$, the respective Jones and Mueller-Jones matrices representing the " $i$ " element. Thus, the Jones vector of the light pencil emerging from each element is given by

$$
\varepsilon_{i}^{\prime}(t)=\mathbf{T}^{(i)}\left[\sqrt{p_{i}} \varepsilon(t)\right]
$$


Incoming radiation

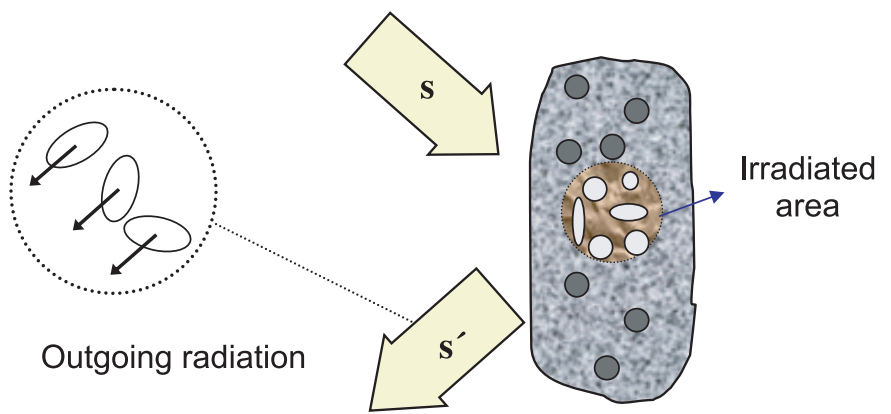

Fig. 10. (Color online) Physical transformation of Stokes vectors.

and the corresponding Stokes vector is given by

$$
\begin{aligned}
\mathbf{s}_{i}^{\prime} & =\mathbf{L}\left\langle\boldsymbol{\varepsilon}_{i}^{\prime} \otimes{\boldsymbol{\varepsilon}^{\prime}}_{i}^{*}\right\rangle=\mathbf{L}\left\langle\left(\mathbf{T}^{(i)} \sqrt{p_{i}} \boldsymbol{\varepsilon}\right) \otimes\left(\mathbf{T}^{(i)} \sqrt{p_{i}} \boldsymbol{\varepsilon}\right)^{*}\right\rangle \\
& =\mathbf{L}\left\langle p_{i}\left(\mathbf{T}^{(i)} \otimes \mathbf{T}^{(i) *}\right)\left(\varepsilon \otimes \varepsilon^{*}\right)\right\rangle \\
& =p_{i} \mathbf{L}\left(\mathbf{T}^{(i)} \otimes \mathbf{T}^{(i) *}\right)\left\langle\left(\boldsymbol{\varepsilon} \otimes \varepsilon^{*}\right)\right\rangle \\
& =p_{i}\left[\mathbf{L}\left(\mathbf{T}^{(i)} \otimes \mathbf{T}^{(i) *}\right) \mathbf{L}^{-1}\right] \mathbf{s} .
\end{aligned}
$$

The polarization state of the complete emerging beam is obtained through the incoherent superposition of the beams emerging from the different elements, resulting in the following Stokes vector

$$
\mathbf{s}^{\prime}=\sum_{i} \mathbf{s}_{i}^{\prime}=\left(\sum_{i} p_{i} \mathbf{N}^{(i)}\right) \mathbf{s}=\mathbf{M} \mathbf{s}
$$

where

$$
\begin{array}{r}
\mathbf{M} \equiv\left(\sum_{i} p_{i} \mathbf{N}^{(i)}\right), \quad \mathbf{N}^{(i)} \equiv \mathbf{L}\left(\mathbf{T}^{(i)} \otimes \mathbf{T}^{(i) *}\right) \mathbf{L}^{-1}, \\
p_{i} \geqslant 0, \quad \sum_{i} p_{i}=1 .
\end{array}
$$

We have obtained this result by considering the optical system as composed of a set of parallel elements, but it is worth pointing out that the same result is obtained by considering the system as an ensemble, so that each realization " $i$ ", characterized by a well-defined Jones matrix $\mathbf{T}^{(i)}$, occurs with a probability $p_{i}$. Therefore, we can consider the optical system as composed of such an ensemble [165]. We will refer to ensemble averages by means of

$$
\langle x\rangle_{e} \equiv \sum_{i} p_{i} x^{(i)} ; \quad p_{i} \geqslant 0, \quad \sum_{i} p_{i}=1
$$

so that

$$
\mathbf{M}=\langle\mathbf{N}\rangle_{e}=\mathbf{L}\left\langle\mathbf{T} \otimes \mathbf{T}^{*}\right\rangle_{e} \mathbf{L}^{-1},
$$

or, in components [165],

$$
m_{k l}=\left\langle n_{k l}\right\rangle_{e}=\frac{1}{2}\left\langle\operatorname{tr}\left(\boldsymbol{\sigma}_{k} \mathbf{T} \boldsymbol{\sigma}_{l} \mathbf{T}^{+}\right)\right\rangle_{e}, \quad k, l=0,1,2,3 .
$$

\subsection{Coherency matrix associated with a Mueller matrix and covariance conditions}

A high degree of knowledge of the relationships between the elements of a Mueller matrix is important for analyzing the information contained in such matrices obtained from experimental measurements. As we have indicated above, contributions of various authors who have dealt with this subject provide several sets of constraining conditions for the elements of Mueller matrices $[130,164,167,170,172,174,176,177]$. As we will see, the non-negativity of the four eigenvalues of the Hermitian matrix $\mathbf{H}$ associated with a Mueller matrix $\mathbf{M}$ plays a fundamental role in the general characterization of Mueller matrices. This criterion was introduced by Cloude [161] and derives directly from the ensemble model. Another criterion which must be added in order to complete the mathematical characterization of Mueller matrices, is that the Mueller matrix of any passive optical system must satisfy the "transmittance condition", i.e. $g_{f} \leqslant 1$, where $g_{f}$ is the maximum transmittance (gain) with regard to all possible incident polarization states [124].

As a step previous to the definition of the coherency matrix associated with a Mueller matrix, we introduce the following notation for the elements of $\mathbf{T} t_{0} \equiv t_{11}, t_{1} \equiv t_{12}$, $t_{2} \equiv t_{21}, t_{3} \equiv t_{22}$, which allows the matrix $\left\langle\mathbf{T} \otimes \mathbf{T}^{*}\right\rangle_{e}$ to be written in the following manner

$$
\left\langle\mathbf{T} \otimes \mathbf{T}^{*}\right\rangle_{e}=\left[\begin{array}{cccc}
\left\langle t_{0} t_{0}^{*}\right\rangle_{e} & \left\langle t_{0} t_{1}^{*}\right\rangle_{e} & \left\langle t_{1} t_{0}^{*}\right\rangle_{e} & \left\langle t_{1} t_{1}^{*}\right\rangle_{e} \\
\left\langle t_{0} t_{2}^{*}\right\rangle_{e} & \left\langle t_{0} t_{3}^{*}\right\rangle_{e} & \left\langle t_{1} t_{2}^{*}\right\rangle_{e} & \left\langle t_{1} t_{3}^{*}\right\rangle_{e} \\
\left\langle t_{2} t_{0}^{*}\right\rangle_{e} & \left\langle t_{2} t_{1}^{*}\right\rangle_{e} & \left\langle t_{3} t_{0}^{*}\right\rangle_{e} & \left\langle t_{3} t_{1}^{*}\right\rangle_{e} \\
\left\langle t_{2} t_{2}^{*}\right\rangle_{e} & \left\langle t_{2} t_{3}^{*}\right\rangle_{e} & \left\langle t_{3} t_{2}^{*}\right\rangle_{e} & \left\langle t_{3} t_{3}^{*}\right\rangle_{e}
\end{array}\right],
$$

where, by attending to the subscripts, we observe that the elements can be suitably reordered to construct the matrix $\mathbf{H}[121,168,178]$ whose elements are defined as

$$
h_{k l} \equiv \frac{1}{2}\left\langle t_{k} t_{l}^{*}\right\rangle_{e}, \quad k, l=0,1,2,3 .
$$

This matrix was first introduced by Simon [121], who distinguished between two cases $\mathbf{H}$ positive semidefinite and $\mathbf{H}$ negative definite [178]. As we will see in next paragraph this last case never corresponds to physical Mueller matrices.

This definition of the Hermitian matrix $\mathbf{H}$ shows that its elements are, in fact, the second-order moments of the variables $t_{k} / \sqrt{2}$. A necessary and sufficient condition for a Hermitian matrix $\mathbf{H}$ to be a matrix of second-order moments is that $\mathbf{H}$ be positive semidefinite [88]. Moreover, a Hermitian matrix $\mathbf{H}$ is positive semidefinite if, and only if, its eigenvalues $\lambda_{i}$ are non-negative [103].

Through symbolic calculus it is possible to obtain the very complicated algebraic expressions for the four eigenvalues of $\mathbf{H}$ and, hence, to express the conditions of their non-negativity.

Another equivalent set of conditions, whose algebraic expressions are simpler, is derived from the non-negativity of four nested principal minors of $\mathbf{H}$ [168]. This explicit formulation of the conditions could be useful in order to show some properties of the structure of Mueller matrices, 
$\mathbf{H}=\frac{1}{4}\left(\begin{array}{cccc}m_{00}+m_{01}+m_{10}+m_{11} & m_{02}+m_{12}+i\left(m_{03}+m_{13}\right) & m_{20}+m_{21}-i\left(m_{30}+m_{31}\right) & m_{22}+m_{33}+i\left(m_{23}-m_{32}\right) \\ m_{02}+m_{12}-i\left(m_{03}+m_{13}\right) & m_{00}-m_{01}+m_{10}-m_{11} & m_{22}-m_{33}-i\left(m_{23}+m_{32}\right) & m_{20}-m_{21}-i\left(m_{30}-m_{31}\right) \\ m_{20}+m_{21}+i\left(m_{30}+m_{31}\right) & m_{22}-m_{33}+i\left(m_{23}+m_{32}\right) & m_{00}+m_{01}-m_{10}-m_{11} & m_{02}-m_{12}+i\left(m_{03}-m_{13}\right) \\ m_{22}+m_{33}-i\left(m_{23}-m_{32}\right) & m_{20}-m_{21}+i\left(m_{30}-m_{31}\right) & m_{02}-m_{12}-i\left(m_{03}-m_{13}\right) & m_{00}-m_{01}-m_{10}+m_{11},\end{array}\right)$

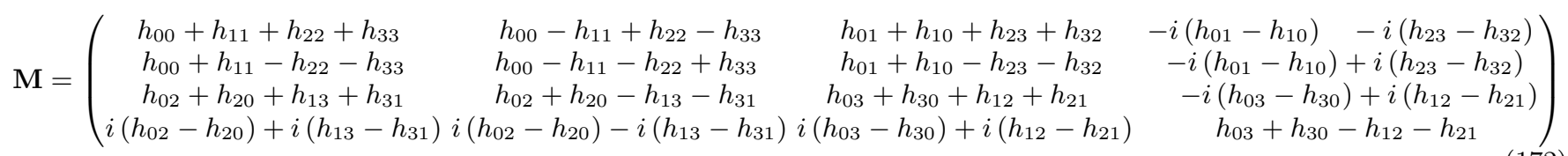

to analyze polarimetric measurements and to study physical models. To obtain convenient expressions of these constraining conditions, it is useful to write the $h_{k l}$ elements in terms of statistical parameters

$$
h_{k l} \equiv \mu_{k l} \sigma_{k} \sigma_{l}
$$

where

$$
\begin{aligned}
\sigma_{k}^{2}=h_{k k} & =\left\langle\left(\frac{1}{\sqrt{2}} t_{k}\right)\left(\frac{1}{\sqrt{2}} t_{k}\right)^{*}\right\rangle_{e}=\frac{1}{2}\left\langle\left|t_{k}\right|^{2}\right\rangle_{e}, \\
\mu_{k l} & =\frac{h_{k l}}{\sigma_{k} \sigma_{l}}\left(\mu_{k l} \equiv \rho_{k l} e^{i \beta_{k l}}\right) .
\end{aligned}
$$

Thus, $\mathbf{H}$ can be written as follows

$$
\mathbf{H}=\left[\begin{array}{llll}
\sigma_{0}^{2} & \mu_{01} \sigma_{0} \sigma_{1} & \mu_{02} \sigma_{0} \sigma_{2} & \mu_{03} \sigma_{0} \sigma_{3} \\
\mu_{01}^{*} \sigma_{0} \sigma_{1} & \sigma_{1}^{2} & \mu_{12} \sigma_{1} \sigma_{2} & \mu_{13} \sigma_{1} \sigma_{3} \\
\mu_{02}^{*} \sigma_{0} \sigma_{2} & \mu_{12}^{*} \sigma_{1} \sigma_{2} & \sigma_{2}^{2} & \mu_{23} \sigma_{2} \sigma_{3} \\
\mu_{03}^{*} \sigma_{0} \sigma_{3} & \mu_{13}^{*} \sigma_{1} \sigma_{3} & \mu_{23}^{*} \sigma_{2} \sigma_{3} & \sigma_{3}^{2}
\end{array}\right] .
$$

From the non-negativity of four nested principal minors, we can write the set of necessary and sufficient conditions (hereafter called "covariance conditions") for a Hermitian matrix $\mathbf{H}$ to be a matrix of second-order moments [168]

$$
\begin{gathered}
h_{00} \geqslant 0 ; \\
1 \geqslant \rho_{01} ; \\
1+2 \rho_{01} \rho_{12} \rho_{02} \cos \left(\beta_{01}+\beta_{12}-\beta_{02}\right) \geqslant \rho_{01}^{2}+\rho_{12}^{2}+\rho_{02}^{2} ; \\
\operatorname{det}(\mathbf{H})= \\
+2 \rho_{12} \rho_{23} \rho_{13} \cos \left(\beta_{12}+\beta_{23}-\beta_{13}\right) \\
+2 \rho_{02} \rho_{23} \rho_{03} \cos \left(\beta_{02}+\beta_{23}-\beta_{03}\right) \\
+2 \rho_{01} \rho_{13} \rho_{03} \cos \left(\beta_{01}+\beta_{13}-\beta_{03}\right) \\
+2 \rho_{01} \rho_{12} \rho_{02} \cos \left(\beta_{01}+\beta_{12}-\beta_{02}\right) \\
-2 \rho_{01} \rho_{02} \rho_{13} \rho_{23} \cos \left(\beta_{01}-\beta_{02}+\beta_{13}-\beta_{23}\right) \\
-2 \rho_{01} \rho_{03} \rho_{12} \rho_{23} \cos \left(\beta_{01}-\beta_{03}+\beta_{12}+\beta_{23}\right) \\
-2 \rho_{02} \rho_{03} \rho_{12} \rho_{13} \cos \left(\beta_{02}-\beta_{03}-\beta_{12}+\beta_{13}\right) \\
+\rho_{01}^{2} \rho_{23}^{2}+\rho_{02}^{2} \rho_{13}^{2}+\rho_{03}^{2} \rho_{12}^{2} \\
-\rho_{01}^{2}-\rho_{02}^{2}-\rho_{03}^{2}-\rho_{12}^{2}-\rho_{13}^{2}-\rho_{23}^{2} \geqslant 0 .
\end{gathered}
$$

It should be noted that, regardless of the fact that the above four inequalities provide a set of sufficient conditions; all the fifteen principal minors of $\mathbf{H}$ are nonnegative.

The pure case occurs when the following nine independent conditions are satisfied

$$
\begin{aligned}
& \rho_{i j}=1 \quad(i, j=0,1,2,3), \\
& \beta_{01}+\beta_{12}=\beta_{02}, \beta_{12}+\beta_{23}=\beta_{13}, \quad \beta_{02}+\beta_{23}=\beta_{03} .
\end{aligned}
$$

This "deterministic" case corresponds to a Mueller-Jones matrix $\mathbf{N}(\mathbf{H})$ [168].

As a basis for the expansion of $\mathbf{H}$, let us now consider the following set of Hermitian trace-orthogonal matrices $[161,179]$

$$
\mathbf{E}_{i j}=\boldsymbol{\sigma}_{i} \otimes \boldsymbol{\sigma}_{j}(i, j=0,1,2,3),
$$

which are defined as direct products of the $\boldsymbol{\sigma}_{i}$ matrices used for the expansion of the 2D coherency matrix (i.e. the Pauli matrices plus the identity matrix).

As occurs with the basis used for the expansions of the $2 \times 2$ and $3 \times 3$ coherency matrices, these matrices $\mathbf{E}_{i j}$ are Hermitian $\mathbf{E}_{i j}=\mathbf{E}_{i j}^{+}$; trace-orthogonal $\operatorname{tr}\left(\mathbf{E}_{i j} \mathbf{E}_{k l}\right)=$ $4 \delta_{i k} \delta_{j l}$, and satisfy $\mathbf{E}_{i j}^{2}=\mathbf{D}(1,1,1,1)$. Thus, these matrices are unitary and, except for $\mathbf{E}_{00}=\mathbf{D}(1,1,1,1)$, are traceless. This basis allows $\mathbf{H}$ to be expressed as a linear combination of $\mathbf{E}_{i j}$

$$
\mathbf{H}=\frac{1}{4} \sum_{i, j=0}^{3} m_{i j} \mathbf{E}_{i j}, \quad m_{i j}=\operatorname{tr}\left(\mathbf{E}_{i j} \mathbf{H}\right),
$$

where the sixteen real coefficients $m_{i j}$ are just the elements of the Mueller matrix associated with the "coherency matrix" H. It should be noted that the term "coherency matrix" is used by Cloude [161], van der Mee [169] and other authors to refer to other Hermitian matrices defined from $\mathbf{M}$ in a different way.

The formulation given by equation (170), together with the non-negativity of $\mathbf{H}$ has been considered by Aiello, Puentes and Woerdman [180], who have analyzed the connection between classical polarization optics and quantum mechanics of two level systems. The explicit relations between the coherency matrix $\mathbf{H}$ and its corresponding Mueller matrix $\mathbf{M}$ are $[121,168,178]$.

$$
\text { see equations (171,172) above }
$$


The constraining inequalities between $m_{i j}$ (derived from the non-negativity of the eigenvalues of $\mathbf{H}$ ) are of higher degree of complexity than in the 2D and 3D Stokes parameters, and involve the invariants $\operatorname{tr} \mathbf{H}, \operatorname{tr}\left(\mathbf{H}^{2}\right), \operatorname{tr}\left(\mathbf{H}^{3}\right)$ and $\operatorname{tr}\left(\mathbf{H}^{4}\right)$. Leaving aside the fact that the $4 \times 4$ coherency matrix $\mathbf{H}$ represents the polarimetric properties of material media, from $\mathbf{H}$ we can reproduce easily the coherency matrices $\boldsymbol{\Phi}$ and $\mathbf{R}$ representing the polarimetric properties of light in the respective 2D and 3D models. Therefore, by eliminating the corresponding extra components of the variables $t_{i}$, the $2 \mathrm{D}$ model arises as the upper-left $2 \times 2$ matrix, by placing

$$
\begin{aligned}
& s_{0}=m_{00}+m_{10}, \quad s_{1}=m_{01}+m_{11}, \\
& s_{2}=m_{02}+m_{12}, \quad s_{3}=-m_{03}-m_{13} .
\end{aligned}
$$

The restriction to the $3 \mathrm{D}$ model can be straightforward performed but, as occurs in the restriction $3 \mathrm{D}-2 \mathrm{D}$, the explicit expressions for the 3D Stokes parameters in terms of $m_{i j}$ are more complicated because of the mathematical asymmetry of the Gell-Mann matrices with respect to the $\mathbf{E}_{i j}$ matrices.

The expansion given by equation (170) provides a fundamental significance for the elements of the Mueller matrix, deeper than their simple phenomenological significance as coefficients of the linear transformation of Stokes vectors. We see that the relation between the complex correlation matrix $\mathbf{H}$ and its corresponding Mueller matrix is, in fact, analogous to the relation between the $2 \times 2$ coherency matrix and its corresponding Stokes parameters. Thus, leaving aside the physical meaning of the elements $m_{i j}$ of the Mueller matrix, they can be mathematically considered as 4D Stokes parameters. This shows the symmetry of the operators representing polarization quantities for both light and material media.

Recently, Sudha, Gopala Rao, Usha Devi and Rajagopal [181] have emphasized the mathematical analogy between the formulation of the coherency matrix $\mathbf{H}$, derived from the ensemble criterion, and the positive operator valued measures (POVM) of the quantum density matrix and have proposed a way of realizing different types of Mueller devices.

\subsection{Transmittance conditions}

The covariance conditions have been obtained from the construction of the Mueller matrix as the average given by equation (159a) — or equation $(161)$ - where $\mathbf{T}^{(i)}$ are $2 \times 2$ complex matrices, but without taking into account the conditions derived from $\mathbf{T}^{(i)}$ being passive Jones matrices. Thus, in order to complete the set of conditions for a $4 \times 4$ real matrix to be a Mueller matrix, it is necessary to consider the conditions derived from the fact that all the pure Mueller matrices of the elements involved in the average satisfy the transmittance condition.

The direct application to a Mueller matrix of the condition of passivity (i.e. its transmittance never exceeds the value 1) leads to the well-known condition $[124,126]$

$$
m_{00}+\left(m_{01}^{2}+m_{02}^{2}+m_{03}^{2}\right)^{1 / 2} \leqslant 1 .
$$

Nevertheless, through calculations based on two main hypotheses: (1) the system is considered as an ensemble (the Mueller matrix is given by an ensemble average of pure Mueller matrices) and (2) each single realization is a passive system (i.e. its pure Mueller matrix satisfies the transmittance condition), the following pair of conditions can be demonstrated [168]

$$
\begin{gathered}
g_{f} \leqslant 1, \quad g_{r} \leqslant 1 \\
g_{f} \equiv m_{00}+\left(m_{01}^{2}+m_{02}^{2}+m_{03}^{2}\right)^{1 / 2}, \\
g_{r} \equiv m_{00}+\left(m_{10}^{2}+m_{20}^{2}+m_{30}^{2}\right)^{1 / 2} .
\end{gathered}
$$

Thus, the procedure followed to construct the general Mueller matrices as a convex sum (or as an ensemble average) of passive pure Mueller matrices, leads to the fact that any Mueller matrix must satisfy, not only the "forward transmittance condition"

$$
m_{00}+\left(m_{01}^{2}+m_{02}^{2}+m_{03}^{2}\right)^{1 / 2} \leqslant 1,
$$

but also the "reverse transmittance condition"

$$
m_{00}+\left(m_{10}^{2}+m_{20}^{2}+m_{30}^{2}\right)^{1 / 2} \leqslant 1 .
$$

The origin of these names derives from the fact that $g_{r}$ represents the maximum transmittance for light which passes through the system in the reverse direction.

A good example for analyzing these properties of Mueller matrices is the following matrix associated with a "transparent ideal circular polarizer"

$$
\left[\begin{array}{llll}
1 & 0 & 0 & 0 \\
0 & 0 & 0 & 0 \\
0 & 0 & 0 & 0 \\
1 & 0 & 0 & 0
\end{array}\right] .
$$

It is easy to check that this matrix satisfies the covariance conditions as well as the forward transmittance condition, but it does not satisfy the reverse transmittance condition. This is consistent with the fact that this matrix cannot be obtained as an average of pure Mueller matrices and, hence, it is impossible to realize it and is not a Mueller matrix.

\subsection{Characterization theorem for Mueller matrices}

Let us now consider the starting premises for obtaining the characterization of Mueller matrices:

(1) a $2 \times 2$ complex matrix is a Jones matrix if, and only if, it satisfies the transmittance condition given by equation (107);

(2) a Mueller-Jones matrix is defined as a $4 \times 4$ real matrix which can be derived from a Jones matrix. Therefore, a Mueller-Jones matrix can be expressed by means of equation (105) in function of its corresponding Jones matrix. In consequence, a Mueller-Jones matrix satisfies the transmittance condition given by equation (110), which can also be expressed by means of equation (112); 


$\mathbf{M}=\frac{1}{2}\left[\begin{array}{c|c|c|c}\sigma_{0}^{2}+\sigma_{1}^{2}+\sigma_{2}^{2}+\sigma_{3}^{2} & \sigma_{0}^{2}-\sigma_{1}^{2}-\sigma_{2}^{2}+\sigma_{3}^{2} & \begin{array}{c}2 \rho_{02} \sigma_{0} \sigma_{2} c_{02} \\ +2 \rho_{23} \sigma_{2} \sigma_{3} c_{23}\end{array} & \begin{array}{c}2 \rho_{01} \sigma_{0} \sigma_{1} s_{01} \\ -2 \rho_{23} \sigma_{2} \sigma_{3} s_{23}\end{array} \\ \hline \sigma_{0}^{2}+\sigma_{1}^{2}-\sigma_{2}^{2}-\sigma_{3}^{2} & \sigma_{0}^{2}-\sigma_{1}^{2}-\sigma_{2}^{2}+\sigma_{3}^{2} & 2 \rho_{01} \sigma_{0} \sigma_{1} c_{01} & 2 \rho_{01} \sigma_{0} \sigma_{1} s_{01} \\ -2 \rho_{23} \sigma_{2} \sigma_{3} c_{23} & +2 \rho_{23} \sigma_{2} \sigma_{3} s_{23} \\ \hline 2 \rho_{02} \sigma_{0} \sigma_{2} c_{02} & 2 \rho_{02} \sigma_{0} \sigma_{2} c_{02} & 2 \rho_{03} \sigma_{0} \sigma_{2} c_{02} & +2 \rho_{03} \sigma_{0} \sigma_{3} s_{03} \\ +2 \rho_{12} \sigma_{1} \sigma_{2} c_{13} & -2 \rho_{13} \sigma_{1} \sigma_{3} c_{13} & +2 \rho_{12} \sigma_{2} \sigma_{3} c_{12} & -2 \rho_{12} \sigma_{1} \sigma_{2} s_{12} \\ \hline-2 \rho_{02} \sigma_{0} \sigma_{3} s_{02} & -2 \rho_{02} \sigma_{0} \sigma_{3} s_{02} & -2 \rho_{03} \sigma_{0} \sigma_{1} s_{03} & 2 \rho_{03} \sigma_{0} \sigma_{1} c_{03} \\ -2 \rho_{13} \sigma_{1} \sigma_{3} s_{13} & +2 \rho_{13} \sigma_{3} \sigma_{2} s_{12} & -2 \rho_{23} \sigma_{2} \sigma_{3} s_{23} & +2 \rho_{12} \sigma_{1} \sigma_{2} c_{12}\end{array}\right]$

(3) the set of Mueller matrices is defined by all the $4 \times 4$ real matrices which can be obtained by means of a convex sum (or by an ensemble average) of MuellerJones matrices;

(4) characterization theorem. The results of the previous sections lead to the following statement: "A real $4 \times 4$ matrix $\mathbf{M}$ is a Mueller matrix if, and only if, the four eigenvalues of $\mathbf{H}(\mathbf{M})$ are non-negative and $\mathbf{M}$ satisfies the transmittance conditions given by equation (176) and equation (177)".

In terms of simpler algebraically explicit expressions, this statement can be formulated as: "a real $4 \times 4$ matrix is a Mueller matrix if, and only if, it satisfies the following six inequalities: the four covariance conditions given by equation (167) and the two transmittance conditions given by equations (176) and (177)".

Evidently, in all the previous paragraphs we use the terms "Jones matrices" and "Mueller matrices" to refer to physical Jones and Mueller matrices respectively. Despite the reiteration, we consider it appropriate to emphasize this in order to avoid any confusion due to the several terms and definitions used in the literature [182].

When a Mueller matrix is obtained from an experimental measurement, we of course expect that, leaving aside experimental errors, it corresponds to a real optical system. However, it is of interest to consider the above general constraints in order to know the range of possible expectations as well as if the results obtained are consistent. Furthermore, the knowledge of a general characterization of Mueller matrices allows us to interpret the results in terms of parameters with specific physical meaning.

A useful way to explicitly show most of the covariance conditions (although not all) in the expression of the Mueller matrix $\mathbf{M}$, is to write it in function of the statistical parameters, so that we get

see equation (179) above

where $c_{i j}=\cos \beta_{i j}, \mathrm{~s}_{i j}=\sin \beta_{i j}$.
From equation (179) we observe that the following Stokes vectors can be constructed in terms of the elements of $\mathbf{M}$

$$
\begin{aligned}
& \mathbf{s}^{(01)} \equiv\left[\begin{array}{c}
m_{00}+m_{10} \\
m_{01}+m_{11} \\
m_{02}+m_{12} \\
m_{03}+m_{13}
\end{array}\right]=\left[\begin{array}{c}
\sigma_{0}^{2}+\sigma_{1}^{2} \\
\sigma_{0}^{2}-\sigma_{1}^{2} \\
2 \rho_{01} \sigma_{0} \sigma_{1} c_{01} \\
2 \rho_{01} \sigma_{0} \sigma_{1} s_{01}
\end{array}\right], \\
& \mathbf{s}^{(23)} \equiv\left[\begin{array}{c}
m_{00}-m_{10} \\
m_{01}-m_{11} \\
m_{02}-m_{12} \\
m_{03}-m_{13}
\end{array}\right]=\left[\begin{array}{c}
\sigma_{2}^{2}+\sigma_{3}^{2} \\
\sigma_{2}^{2}-\sigma_{3}^{2} \\
2 \rho_{23} \sigma_{2} \sigma_{3} c_{23} \\
2 \rho_{23} \sigma_{2} \sigma_{3} s_{23}
\end{array}\right] \text {, } \\
& \mathbf{s}^{(02)} \equiv\left[\begin{array}{c}
m_{00}+m_{01} \\
m_{10}+m_{11} \\
m_{20}+m_{21} \\
m_{30}+m_{31}
\end{array}\right]=\left[\begin{array}{c}
\sigma_{0}^{2}+\sigma_{2}^{2} \\
\sigma_{0}^{2}-\sigma_{2}^{2} \\
2 \rho_{02} \sigma_{0} \sigma_{2} c_{02} \\
-2 \rho_{02} \sigma_{0} \sigma_{2} s_{02}
\end{array}\right] \text {, } \\
& \mathbf{s}^{(13)} \equiv\left[\begin{array}{c}
m_{00}-m_{01} \\
m_{10}-m_{11} \\
m_{20}-m_{21} \\
m_{30}-m_{31}
\end{array}\right]=\left[\begin{array}{c}
\sigma_{1}^{2}+\sigma_{3}^{2} \\
\sigma_{1}^{2}-\sigma_{3}^{2} \\
2 \rho_{13} \sigma_{1} \sigma_{3} c_{13} \\
-2 \rho_{13} \sigma_{1} \sigma_{3} s_{13}
\end{array}\right] \text {, } \\
& \mathbf{s}^{(03)} \equiv\left[\begin{array}{c}
m_{00}+m_{11} \\
m_{10}+m_{01} \\
m_{22}+m_{33} \\
m_{23}-m_{32}
\end{array}\right]=\left[\begin{array}{c}
\sigma_{0}^{2}+\sigma_{3}^{2} \\
\sigma_{0}^{2}-\sigma_{3}^{2} \\
2 \rho_{03} \sigma_{0} \sigma_{3} c_{03} \\
2 \rho_{03} \sigma_{0} \sigma_{3} s_{03}
\end{array}\right] \text {, } \\
& \mathbf{s}^{(12)} \equiv\left[\begin{array}{c}
m_{00}-m_{11} \\
m_{10}-m_{01} \\
m_{22}-m_{33} \\
m_{23}+m_{32}
\end{array}\right]=\left[\begin{array}{c}
\sigma_{1}^{2}+\sigma_{2}^{2} \\
\sigma_{1}^{2}-\sigma_{2}^{2} \\
2 \rho_{12} \sigma_{1} \sigma_{2} c_{12} \\
-2 \rho_{12} \sigma_{1} \sigma_{2} s_{12}
\end{array}\right] \text {. }
\end{aligned}
$$

These expressions are formally identical to those corresponding to certain Stokes vectors where the magnitudes $\sigma_{k}^{2}$ are the mean quadratic amplitudes and $\rho_{k l}, \beta_{k l}$ are the respective modulus and arguments of the degrees of coherence.

It is important to point out that the first four Stokes vectors given by equation (180) can be obtained as

$$
\begin{aligned}
& \mathbf{s}^{(01)}=\mathbf{M}^{r}(1,1,0,0)^{T}, \mathbf{s}^{(23)}=\mathbf{M}^{r}(1,-1,0,0)^{T}, \\
& \mathbf{s}^{(02)}=\mathbf{M}(1,1,0,0)^{T}, \quad \mathbf{s}^{(13)}=\mathbf{M}(1,-1,0,0)^{T}
\end{aligned}
$$

whereas the last two $\mathbf{s}^{(03)}$ and $\mathbf{s}^{(12)}$, cannot be obtained by means of direct transformations of Stokes vectors. This 
property arises from the peculiar structure of Mueller matrices and not from the fact that they transform Stokes vectors into Stokes vectors.

Obviously, any Mueller matrix is a Stokes matrix. The converse statement is not true because there are Stokes matrices such as $\mathbf{X} \equiv \mathbf{D}(1,0,1,1)$, whose corresponding vector $\mathbf{s}^{(03)}(\mathbf{X})=(1,0,2,0)^{T}$ is not a Stokes vector. Another counterexample is the Stokes matrix $\mathbf{Y} \equiv \mathbf{D}(1,1,1,0)$, whose corresponding vector $\mathbf{s}^{(12)}(\mathbf{Y})=$ $(0,0,1,0)^{T}$ is not a Stokes vector.

Since any Mueller matrix is a Stokes matrix, we conclude that the numerous inequalities quoted in the literature for Stokes matrices are all deducible, as necessary conditions, from the above general characterization of Mueller matrices.

\subsection{The purity criterion for Mueller matrices and the degree of polarimetric purity of material media}

The study of the necessary and sufficient conditions for a Mueller matrix to be a pure Mueller matrix is an important subject dealt with by several authors $[121,122,125,164-167,178,182-188]$.

We can now analyze this subject in the light of the properties of the matrix $\mathbf{H}$.

As with the $2 \times 2$ and $3 \times 3$ coherency matrices, we consider here the Euclidean norms of $\mathbf{H}$ and $\mathbf{M}$

$$
\begin{aligned}
\|\mathbf{H}\|_{2} & \equiv\left(\sum_{i, j=0}^{3}\left|h_{i j}\right|^{2}\right)^{1 / 2}=\left[\operatorname{tr}\left(\mathbf{H}^{+} \mathbf{H}\right)\right]^{1 / 2}=\left[\operatorname{tr}\left(\mathbf{H}^{2}\right)\right]^{1 / 2} \\
\|\mathbf{M}\|_{2} & \equiv\left(\sum_{i, j=0}^{3} m_{i j}^{2}\right)^{1 / 2}=\left[\operatorname{tr}\left(\mathbf{M}^{T} \mathbf{M}\right)\right]^{1 / 2},
\end{aligned}
$$

and we define the norm

$$
\|\mathbf{H}\|_{0} \equiv \operatorname{tr} \mathbf{H}=\|\sqrt{\mathbf{H}}\|_{2}^{2} .
$$

It is easy to show that these norms satisfy the following relations

$$
\begin{aligned}
\|\mathbf{H}\|_{2}^{2} & =\frac{1}{4}\|\mathbf{M}\|_{2}^{2} \\
\|\mathbf{H}\|_{0} & =m_{00} \\
\frac{1}{4}\|\mathbf{H}\|_{0}^{2} & \leqslant\|\mathbf{H}\|_{2}^{2} \leqslant\|\mathbf{H}\|_{0}^{2} .
\end{aligned}
$$

The last relation shows that $\|\mathbf{H}\|_{2}^{2}=\|\mathbf{H}\|_{0}^{2}$, or equivalently $\sum_{i, j=0}^{3} m_{i j}^{2}=4 m_{00}^{2}$, is a necessary and sufficient condition for $\mathbf{H}$ to have only a nonzero eigenvalue and, in consequence, is a necessary and sufficient condition for a $4 \times 4$ coherency matrix to correspond to a Mueller-Jones matrix [168]. The other limit $\|\mathbf{H}\|_{2}^{2}=\frac{1}{4}\|\mathbf{H}\|_{0}^{2}$ is reached when the system is composed of an equiprobable mixture of pure elements.
The inequality $\|\mathbf{H}\|_{2}^{2} \leqslant\|\mathbf{H}\|_{0}^{2}$ was first shown by Fry and Kattawar [164] in terms of the elements of the Mueller matrix, and they found that the equality

$$
\operatorname{tr}\left(\mathbf{M}^{T} \mathbf{M}\right)=4 m_{00}^{2}
$$

is satisfied by any Mueller-Jones matrix. However, the question whether, given a Mueller matrix $\mathbf{M}$, this is a sufficient condition for $\mathbf{M}$ to be a Mueller-Jones matrix has been a subject discussed in various papers $[121,122,165,173,176,178,182,186,187,189,190]$. It is clear that, under the premise that a Mueller matrix is expressible as a convex sum of Mueller-Jones matrices, we can state: "given a Mueller matrix M, equation (187) is a sufficient condition for $\mathbf{M}$ to be a Mueller-Jones matrix". All the arguments contrary to this statement arise from considering real $4 \times 4$ matrices which do not satisfy the eigenvalue conditions or the transmittance conditions $[173,178,182,186,189,190]$.

The degree of polarimetric purity $P_{(4)}$ of a material sample is defined as $[41,131,168]$

$$
P_{(4)}=\left|\frac{1}{3}\left(\frac{4 \operatorname{tr}\left(\mathbf{H}^{2}\right)}{(\operatorname{tr} \mathbf{H})^{2}}-1\right)^{1 / 2}\right|
$$

This invariant non-dimensional parameter is restricted to the interval $0 \leqslant P_{(4)} \leqslant 1$. The minimum $P_{(4)}=0$ corresponds to an ideal total depolarizer, characterized by the fact that all eigenvalues of $\mathbf{H}$ are equal, i.e. the medium is composed of an equiprobable mixture of elements, and does not exhibit any polarimetric preference $\left(m_{i j}=0\right.$ except $\left.m_{00}\right)$. The maximum corresponds to a pure system $\left(\lambda_{0}>0, \lambda_{1}=\lambda_{2}=\lambda_{3}=0\right)$.

This fundamental physical quantity $P_{(4)}$ was first introduced by Gil and Bernabéu in the form of a "depolarizing index" in terms of the elements of $\mathbf{M}$ [131]

$$
\begin{aligned}
P_{(4)} & =\left|\left[\frac{1}{3}\left(\frac{\sum_{i, j=0}^{3} m_{i j}^{2}}{m_{00}^{2}}-1\right)\right]^{1 / 2}\right| \\
& =\left|\left[\frac{1}{3}\left(\frac{\operatorname{tr}\left(\mathbf{M}^{T} \mathbf{M}\right)}{m_{00}^{2}}-1\right)\right]^{1 / 2}\right| .
\end{aligned}
$$

In function of both norms of $\mathbf{H}, P_{(4)}$ is expressed as

$$
P_{(4)}=\left|\left[\frac{1}{3}\left(\frac{4\|\mathbf{H}\|_{2}^{2}}{\|\mathbf{H}\|_{0}^{2}}-1\right)\right]^{1 / 2}\right| .
$$

This parameter gives an objective measure of the global polarimetric purity of the system, as well as of its depolarizing power, and provides criteria for the analysis of measured Mueller matrices [58,191,193].

Moreover, the "depolarizance" $D$ of an optical system can be defined as

$$
D \equiv 1-P_{(4)}
$$


This definition is proper because $D$ can be obtained as an average measure of the depolarization produced by the system over all incident pure states [131]. This quantity has been studied by Chipman [194] and compared with another scalar measure of the depolarizing power of a material sample: the "average degree of polarization" $A$. Both measures are defined from averages of the degree of polarization of the exiting states. The difference between these two quantities is particularly significant for systems involving an asymmetric serial arrangement of diattenuators and depolarizers. This is a direct consequence of the fact that, whereas $D$ is an invariant quantity defined from a symmetric average involving exiting states for both direct $(\mathbf{M})$ and reciprocal $\left(\mathbf{M}^{r}\right)$ Mueller matrices, $A$ only takes into account the effects of direct propagation. Although the definition of $A$ seems to be more natural, the symmetric nature of the characteristic properties of the Mueller matrices leads to a simple geometric interpretation of $D$ as a normalized distance from $\mathbf{M}$ to an ideal depolarizer [194].

\subsection{Parallel incoherent decompositions of matrices representing material media}

As with the case of $3 \times 3$ coherency matrices we consider here the possible parallel decompositions of the coherency matrix $\mathbf{H}(\mathbf{M})$ representing the properties of a material sample. Any such physically realizable decomposition requires having the form of a convex linear combination. That is to say, the incident light is shared among the components, so that the emerging pencils are recombined into the whole emerging beam. The convex linear combination of passive components ensures the passive behavior of the whole system.

5.6.1 The spectral decomposition of the coherency matrix of a material medium

Since $\mathbf{H}$ is a positive semidefinite Hermitian matrix, it can be diagonalized through a unitary transformation

$$
\mathbf{H}=\mathbf{U D}\left(\lambda_{0}, \lambda_{1}, \lambda_{2}, \lambda_{3}\right) \mathbf{U}^{+},
$$

where $\mathbf{D}\left(\lambda_{0}, \lambda_{1}, \lambda_{2}, \lambda_{3}\right)$ represents the diagonal matrix composed of the four non-negative eigenvalues ordered so that $0 \leqslant \lambda_{3} \leqslant \lambda_{2} \leqslant \lambda_{1} \leqslant \lambda_{0}$. The columns $\mathbf{u}_{i} \quad(i=0,1,2,3)$ of the $4 \times 4$ unitary matrix $\mathbf{U}$ are the respective unitary, and mutually orthogonal, eigenvectors.

Therefore, $\mathbf{H}$ can be expressed as the following convex linear combination of four rank-1 coherency matrices that represent respective pure systems

$$
\begin{aligned}
& \mathbf{H}=\frac{\lambda_{0}}{\operatorname{tr} \mathbf{H}} \mathbf{U D}(\operatorname{tr} \mathbf{H}, 0,0,0) \mathbf{U}^{+} \\
& +\frac{\lambda_{1}}{\operatorname{tr} \mathbf{H}} \mathbf{U D}(0, \operatorname{tr} \mathbf{H}, 0,0) \mathbf{U}^{+}+\frac{\lambda_{2}}{\operatorname{tr} \mathbf{H}} \mathbf{U D}(0,0, \operatorname{tr} \mathbf{H}, 0) \mathbf{U}^{+} \\
& +\frac{\lambda_{3}}{\operatorname{tr} \mathbf{H}} \mathbf{U D}(0,0,0, \operatorname{tr} \mathbf{H}) \mathbf{U}^{+},
\end{aligned}
$$

where each term in the sum is affected by its corresponding eigenvector $\mathbf{u}_{i}$, so that

$$
\mathbf{H}=\sum_{i=0}^{3} \frac{\lambda_{i}}{\operatorname{tr} \mathbf{H}} \mathbf{H}^{(i)}, \quad \mathbf{H}^{(i)} \equiv(\operatorname{tr} \mathbf{H})\left(\mathbf{u}_{i} \otimes \mathbf{u}_{i}^{+}\right) .
$$

This spectral decomposition shows that any linear system can be considered as a parallel combination of, up to four, pure systems with weights proportional to the eigenvalues of $\mathbf{H}$. Given the physical meaning of the concept of parallel decomposition, it is very important to keep in mind that any physically realizable parallel decomposition must be expressed as a convex linear combination. Several problems and controversies about the properties of Mueller matrices are derived from neglecting this physical requisite.

Due to the practical importance of this kind of decomposition, it should be noted that when an eigenvalue $\lambda_{i}$ has a multiplicity $r \quad(1<r \leqslant 4)$, the eigenvectors of the corresponding invariant $r$-dimensional subspace are not unique and can be chosen arbitrarily as a set of orthonormal vectors covering the corresponding subspace.

Moreover, the statistical nature of $\mathbf{H}$ leads to a probabilistic interpretation of its eigenvalues. This fact has direct consequences in the interpretation of quantities such as the von Neumann entropy and the indices of purity, which will be considered in later sections.

\subsubsection{The trivial decomposition of the coherency matrix of a material medium}

Let us now consider the $4 D$ trivial decomposition of the coherency matrix $\mathbf{H}$ as a convex linear combination of four systems with equal mean transmittances

$$
\begin{aligned}
\mathbf{H}= & \mathbf{U D}\left(\lambda_{0}, \lambda_{1}, \lambda_{2}, \lambda_{3}\right) \mathbf{U}^{+} \\
= & \frac{\lambda_{0}-\lambda_{1}}{\operatorname{tr} \mathbf{H}} \mathbf{A}+2 \frac{\lambda_{1}-\lambda_{2}}{\operatorname{tr} \mathbf{H}} \mathbf{B}^{(1)}+3 \frac{\lambda_{2}-\lambda_{3}}{\operatorname{tr} \mathbf{H}} \mathbf{B}^{(2)} \\
& +4 \frac{\lambda_{3}}{\operatorname{tr} \mathbf{H}} \mathbf{B}^{(3)} \\
\mathbf{A} \equiv & \operatorname{tr} \mathbf{H}\left[\mathbf{U D}(1,0,0,0) \mathbf{U}^{+}\right] \\
\mathbf{B}^{(1)} \equiv & \frac{1}{2} \operatorname{tr} \mathbf{H}\left[\mathbf{U D}(1,1,0,0) \mathbf{U}^{+}\right] \\
\mathbf{B}^{(2)} \equiv & \frac{1}{3} \operatorname{tr} \mathbf{H}\left[\mathbf{U D}(1,1,1,0) \mathbf{U}^{+}\right] \\
\mathbf{B}^{(3)} \equiv & \frac{1}{4} \operatorname{tr} \mathbf{H}\left[\mathbf{U D}(1,1,1,1) \mathbf{U}^{+}\right] .
\end{aligned}
$$

We observe that any state of the material system is polarimetrically equivalent to a convex linear combination of up to four components with equal mean transmittances: a pure component, a "2D unpolarized system", a "3D unpolarized system" and a "4D unpolarized system". 
This result can also be expressed in terms of Mueller matrices as follows

$$
\begin{aligned}
\mathbf{M}= & \frac{\lambda_{0}-\lambda_{1}}{\operatorname{tr} \mathbf{H}} \mathbf{N}+2 \frac{\lambda_{1}-\lambda_{2}}{\operatorname{tr} \mathbf{H}} \mathbf{M}^{(1)}\left(\mathbf{B}^{(1)}\right) \\
& +3 \frac{\lambda_{2}-\lambda_{3}}{\operatorname{tr} \mathbf{H}} \mathbf{M}^{(2)}\left(\mathbf{B}^{(2)}\right)+4 \frac{\lambda_{3}}{\operatorname{tr} \mathbf{H}} \mathbf{M}^{(3)}\left(\mathbf{B}^{(3)}\right) .
\end{aligned}
$$

As expected, it is straightforward to reproduce the trivial decomposition for 3D and 2D coherency matrices.

Moreover, it is clear that, in general, a non-pure system cannot be decomposed into a pure system and a $4 \mathrm{D}$ unpolarized system. As we have observed previously, a $n \times n$ coherency matrix of a mixed state depends, in general, on $n^{2}$ independent real parameters, whereas a $n \times n$ coherency matrix of a pure state depends, in general, on $2 n-1$ independent real parameters. It is obvious that the trivial decomposition of a $n \times n$ coherency matrix $\left(n^{2}\right.$ parameters) into a convex sum of the coherency matrix of a pure state $(2 n-1$ independent real parameters) and a fully random state (1 parameter) is only possible for $n=2$.

The trivial decomposition constitutes the appropriate framework for distinguishing the pure component from the random or noise component of the material sample. It also allows a proper treatment of the measurement errors when it is known that the target under measurement is pure.

5.6.3 The arbitrary decomposition of the coherency matrix of a material medium

Given the simple linear relation between a coherency matrix and its corresponding Mueller matrix, it is possible to classify Mueller Matrices according to the rank of $\mathbf{H}$, leading to the possible "target decompositions" $[113,191,192]$. The methods for decomposing measured Mueller matrices play an important role in several applications of polarimetry because they can be used for different purposes as, for example, to identify elements in the sample and to improve the contrast of images obtained by radar polarimetry $[59,195]$.

As we have demonstrated for $3 \times 3$ coherency matrices, the spectral decomposition is not the only possibility for decomposing a mixed state. It can also be decomposed through the arbitrary decomposition. This decomposition can be applied to $n \times n$ coherency matrices [102] and, in particular, to $\mathbf{H}$. Moreover, the physical possibility of synthesizing experimentally non-pure systems by means of different parallel combinations of pure systems suggests the existence of decompositions other than the spectral decomposition.

Let us consider a non-pure $4 \times 4$ coherency matrix $\mathbf{H}(1<\operatorname{rank}(\mathbf{H}) \leqslant 4)$.

Using the same arguments as for the arbitrary decomposition of $3 \times 3$ coherency matrices, we obtain the following arbitrary decomposition of the coherency matrix into a linear convex combination of pure coherency matrices

$$
\begin{array}{r}
\mathbf{H}=\sum_{i=0}^{3} \frac{l_{i}}{\operatorname{tr} \mathbf{H}} \mathbf{A}^{(i)}, \quad \sum_{i=0}^{3} \frac{l_{i}}{\operatorname{tr} \mathbf{H}}=1, \quad \mathbf{A}^{(i)}=\mathbf{A}^{(i)+}, \\
\operatorname{rank}\left(\mathbf{A}^{(i)}\right)=1, \quad \operatorname{tr} \mathbf{A}^{(i)}=\operatorname{tr} \mathbf{H} .
\end{array}
$$

This arbitrary decomposition can also be expressed as

$$
\mathbf{H}=\sum_{i=0}^{3} \frac{l_{i}}{\operatorname{tr} \mathbf{H}}\left[(\operatorname{tr} \mathbf{H})\left(\mathbf{v}_{i} \otimes \mathbf{v}_{i}^{+}\right)\right], \quad\left|\mathbf{v}_{i}\right|=1,
$$

where $\mathbf{v}_{i}, \mathbf{v}_{j}$ are linearly independent vectors and constitute a generalized orthogonal basis of the subspace generated by the eigenvectors of $\mathbf{H}$ with nonzero eigenvalues. It is evident that the spectral decomposition is a particular case of the arbitrary decomposition. Each pure component can be expressed in function of the corresponding unitary vector $\mathbf{v}_{i}$. Moreover, in order to ensure a convex sum, the coherency matrices of the components have been chosen satisfying $\operatorname{tr} \mathbf{A}^{(i)}=\operatorname{tr} \mathbf{H}=m_{00}$.

When $\operatorname{rank}(\mathbf{H})=4$, any pure system can be considered as a component and, once chosen, the successive choices of the second and third components are restricted by the exigency that the eigenvector with a nonzero eigenvalue of $\mathbf{A}^{(i)}$ belongs to the subspace generated by the eigenvectors with nonzero eigenvalues of the coherency matrix resulting from the previous subtractions. The fourth component is fully determined by the previous choices of the other pure components.

Obviously, as occurs in the spectral decomposition, the number of pure components of the equivalent system is equal to the rank of $\mathbf{H}$.

We see that any non-pure system is polarimetrically equivalent to a parallel combination of one to four pure components with the same mean transmittance $m_{00}$. As in other sections of this paper, "parallel combination" here means that the set of pure elements of the equivalent system are illuminated by exclusive portions of the incident light, so that their respective emerging beams are incoherently superposed.

It is very important to point out that, among these mathematically possible decompositions, only those where all the components satisfy the transmittance condition are physically realizable, i.e. the corresponding maximum gains satisfy $k_{1}^{(i)}\left(\mathbf{A}^{(i)}\right) \leqslant 1$.

These inequalities are not ensured by the conditions $\operatorname{tr} \mathbf{A}^{(i)}=\operatorname{tr} \mathbf{H}$ and, given a component $\mathbf{A}^{(i)}$, different procedures can be followed in order to check the transmittance condition. For instance, by observing the condition in terms of the elements $a_{k l}^{i}$ of $\mathbf{A}^{(i)}$ [168]

$$
\begin{aligned}
g_{f}^{(i)}=g_{r}^{(i)}= & \left(k_{1}^{(i)}\right)^{2} \\
= & \operatorname{tr}(\mathbf{H})+\left[\left(a_{00}^{i}-a_{11}^{i}+a_{22}^{i}-a_{33}^{i}\right)^{2}\right. \\
& \left.\quad+4\left(a_{01}^{i}+a_{23}^{i}\right)\left(a_{01}^{i *}+a_{23}^{i *}\right)\right]^{1 / 2} \leqslant 1,
\end{aligned}
$$


or by checking equation (110), which expresses the transmittance condition in terms of the elements of the pure Mueller matrix $\mathbf{N}^{(i)}\left(\mathbf{A}^{(i)}\right)$.

For many experimental cases where the target under measurement contains a known (or suspected) pure component, the arbitrary decomposition provides a procedure for a proper subtraction of the known component and, thus, to isolate the unknown part of the sample. The procedure can be iterated if there exists more than one known pure component.

Moreover, the arbitrary decomposition has important consequences because it allows the identification of all the possible physically realizable target decompositions. In fact, it provides a method for analyzing measured samples and adjusting the target decomposition in order to obtain improvements in identifying unknown components. When applied to imaging polarimetry, this polarimetric subtraction can be applied to improve strongly the contrast of particular elements of the target with respect to the homogeneous substrate.

The arbitrary decomposition can be expressed in terms of the corresponding Mueller matrices as follows

$$
\mathbf{M}=\sum_{i=0}^{3} \frac{l_{i}}{m_{00}} \mathbf{N}^{(i)}, \quad l_{i} \geqslant 0, \quad \sum_{i=0}^{3} l_{i}=m_{00}, \quad n_{00}^{(i)}=m_{00}
$$

where the Mueller matrix $\mathbf{M}$ of the system is obtained as a convex linear combination of, up to four, Mueller-Jones matrices $\mathbf{N}^{(i)}$.

As we have observed, although the Mueller-Jones matrices $\mathbf{N}^{(i)}$ of the pure components are normalized through the condition $n_{00}^{(i)}=m_{00}$, this does not ensure the fulfillment of the transmittance condition. As an example, let us now consider the Mueller matrix $\mathbf{O}$ of an ideal depolarizer $\left(o_{i j}=0\right.$, except $\left.o_{00}=1\right)$. The canonical spectral decomposition of $\mathbf{H}(\mathbf{O})$ leads to the following decomposition of $\mathbf{O}$

$$
\begin{aligned}
\left(\begin{array}{llll}
1 & 0 & 0 & 0 \\
0 & 0 & 0 & 0 \\
0 & 0 & 0 & 0 \\
0 & 0 & 0 & 0
\end{array}\right)= & \frac{1}{4}\left(\begin{array}{llll}
1 & 1 & 0 & 0 \\
1 & 1 & 0 & 0 \\
0 & 0 & 0 & 0 \\
0 & 0 & 0 & 0
\end{array}\right)+\frac{1}{4}\left(\begin{array}{cccc}
1 & -1 & 0 & 0 \\
1 & -1 & 0 & 0 \\
0 & 0 & 0 & 0 \\
0 & 0 & 0 & 0
\end{array}\right) \\
& +\frac{1}{4}\left(\begin{array}{cccc}
1 & 1 & 0 & 0 \\
-1 & -1 & 0 & 0 \\
0 & 0 & 0 & 0 \\
0 & 0 & 0 & 0
\end{array}\right)+\frac{1}{4}\left(\begin{array}{cccc}
1 & -1 & 0 & 0 \\
-1 & 1 & 0 & 0 \\
0 & 0 & 0 & 0 \\
0 & 0 & 0 & 0
\end{array}\right)
\end{aligned}
$$

Obviously, the components of this linear convex combination do not satisfy the transmittance condition (the factors $1 / 4$ are the coefficients of the convex sum). This example demonstrates that not all the mathematically possible decompositions are physically realizable.
Nevertheless, the transmittance condition is satisfied by all the elements of the following alternative decomposition

$$
\begin{aligned}
\mathbf{O}=\frac{1}{4} \mathbf{N}_{R} & (0,0, \pi / 2)+\frac{1}{4} \mathbf{N}_{R}(0,0,-\pi / 2) \\
& +\frac{1}{4} \mathbf{N}_{R}(\pi / 4,0, \pi)+\frac{1}{4} \mathbf{N}_{R}(\pi / 4, \pi / 2, \pi)
\end{aligned}
$$

whose explicit matricial expression is

$$
\begin{aligned}
\left(\begin{array}{llll}
1 & 0 & 0 & 0 \\
0 & 0 & 0 & 0 \\
0 & 0 & 0 & 0 \\
0 & 0 & 0 & 0
\end{array}\right)= & \frac{1}{4}\left(\begin{array}{cccc}
1 & 0 & 0 & 0 \\
0 & 1 & 0 & 0 \\
0 & 0 & 0 & -1 \\
0 & 0 & 1 & 0
\end{array}\right)+\frac{1}{4}\left(\begin{array}{cccc}
1 & 0 & 0 & 0 \\
0 & 1 & 0 & 0 \\
0 & 0 & 0 & 1 \\
0 & 0 & -1 & 0
\end{array}\right) \\
& +\frac{1}{4}\left(\begin{array}{cccc}
1 & 0 & 0 & 0 \\
0 & -1 & 0 & 0 \\
0 & 0 & -1 & 0 \\
0 & 0 & 0 & 1
\end{array}\right)+\frac{1}{4}\left(\begin{array}{cccc}
1 & 0 & 0 & 0 \\
0 & -1 & 0 & 0 \\
0 & 0 & 1 & 0 \\
0 & 0 & 0 & -1
\end{array}\right)
\end{aligned}
$$

This result constitutes a method for constructing a synthetic perfect depolarizer through the parallel combination of these four pure retarders (two quarter-wave plates and two half-wave plates) with equal cross sections.

\subsection{Geometric maps of the degree of polarization}

Let us consider now some possible graphical representations of the polarimetric effects of material media. As is well-known, the Poincaré sphere plots states of polarization with intensity equal to one, so that the distance to the origin is the degree of polarization. Points on the surface represent pure states, whereas points inside represent nonpure states. The linear interaction of polarized light with material targets produce changes in both intensity and degree of polarization and, therefore, the Poincaré sphere representation (defined for states with intensity equal to 1 ) is not directly applicable for mapping the output states.

As a geometrical and visual tool for analyzing and classifying material samples, based on their effects on the degree of polarization, some authors have studied the "P-image" defined by the points $[2,196-199]$

$$
\frac{1}{s_{0}^{\prime}}\left(s_{1}^{\prime}, s_{2}^{\prime}, s_{3}^{\prime}\right)
$$

which correspond to the output Stokes parameters

$$
\begin{aligned}
\left(s_{0}^{\prime}, s_{1}^{\prime}, s_{2}^{\prime}, s_{3}^{\prime}\right)^{T}=\mathbf{M}\left(s_{0}, s_{1}, s_{2}, s_{3}\right)^{T} & \\
& \left(1=s_{0}^{2} \geqslant s_{1}^{2}+s_{2}^{2}+s_{3}^{2}\right) .
\end{aligned}
$$

The input Stokes vectors map all the points of the solid Poincaré sphere. The distance of each point of the $P$-image to the origin is just its degree of polarization and, in general, varies depending on the corresponding input Stokes vector.

The surface of this solid object is called the "DoP surface" [198] and corresponds to totally polarized input states. 
Moreover, given $\mathbf{M}$, the $I$-image is defined as the surface $[198,199]$

$$
\frac{s_{0}^{\prime}}{\left(s_{1}^{\prime 2}+s_{2}^{\prime 2}+s_{3}^{\prime 2}\right)^{1 / 2}}\left(s_{1}^{\prime}, s_{2}^{\prime}, s_{3}^{\prime}\right) .
$$

These representations allow the classification of the different polarimetric behaviors:

(a) pure homogeneous diattenuators: the $I$-image is not spherical, whereas the surface shape of the $P$-image remains a sphere of radius 1 . Notice that the Mueller matrix is symmetric. Topologically the points are more concentrated around two poles on the surface. The position of these poles is given by the intersection of the maximum and minimum polarizance vectors with the surface. The rectilinear segment connecting these points intersects the image point of the origin of the Poincaré sphere;

(b) pure retarders: none of the objects is deformed (neither morphologically nor topologically). The result is a rotation of both objects with respect to the axis defined by the two antipodal eigenstates of the retarder;

(c) parallel combination (or statistical mixture) of diattenuators: the surface of the $P$-image is deformed and its origin is displaced. The $I$-image is also deformed. When the diattenuators are homogeneous, some symmetries of the objects correspond to the property $\mathbf{M}^{T}=\mathbf{M}$

(d) parallel combination (or statistical mixture) of retarders: the surface of the $P$-image is deformed and the origin remains unchanged. The $I$-image is not deformed;

(e) parallel combination (or statistical mixture) of diattenuators and retarders: the surface of the $P$-image is deformed and its origin is displaced. The $I$-image is also deformed;

A detailed study of these kinds of representations, including plane maps, has been developed by DeBoo, Sasian, and Chipman [198].

\subsection{Indices of purity}

Given the statistical nature of the coherency matrices $\mathbf{H}$ representing material media, we emphasize the importance of obtaining parameters that give a measurement of their polarimetric purity. Usually the degree of purity $P_{(4)}[131,200]$ or, alternatively, the polarization entropy [57], is used as a quantity characterizing the overall purity.

Thus, in a similar manner that to the cases of $2 \times 2$ and $3 \times 3$ coherency matrices representing states of light, the study the "structure purity" of $\mathbf{H}$ requires considering several relative differences between the four eigenvalues of H. Thus, in addition to $P_{(4)}$, three new invariant and nondimensional "indices of purity" can be defined from the eigenvalues of $\mathbf{H}$.
This set of three quantities contains all the information concerning the polarimetric purity. It should be noted that neither $P_{(4)}$ nor the polarization entropy cover all the information mentioned, but they can be calculated from the indices of purity.

At this point, it is important to consider the concept of "polarimetric contrast" introduced by Réfrégier, Roche and Goudail [107] for 3D coherency matrices. These authors have pointed out that for 3D polarization states with Gaussian fluctuations, three invariant quantities are relevant to characterize the contrast in polarimetric imagery. There are several cases where a $3 \times 3$ representation of the coherency matrix is enough for the characterization of the measured polarimetric quantities (e.g. monostatic radar polarimetry). Nevertheless, a complete Mueller polarimetry requires the consideration of the $4 \times 4$ coherency matrix $\mathbf{H}$ and, in consequence, at least four invariant quantities must be considered for a complete characterization of the polarimetric contrast. A proper set of such quantities is constituted by the three indices of purity (defined below) and the transmittance for unpolarized light $m_{00}=\operatorname{tr} \mathbf{H}=\lambda_{0}+\lambda_{1}+\lambda_{2}+\lambda_{3}, \lambda_{i}$ being the eigenvalues of $\mathbf{H}$.

An examination of the expressions of the eigenvalues of $\mathbf{H}$ obtained by algebraic computation shows that it is possible to write them in terms of three non-negative nondimensional parameters

$$
\begin{aligned}
\lambda_{0} & =\frac{1}{4} \operatorname{tr} \mathbf{H}\left(1+P_{2}+2 P_{1}\right), & \lambda_{1} & =\frac{1}{4} \operatorname{tr} \mathbf{H}\left(1+P_{2}-2 P_{1}\right), \\
\lambda_{2} & =\frac{1}{4} \operatorname{tr} \mathbf{H}\left(1-P_{2}+2 P_{3}\right), & \lambda_{3} & =\frac{1}{4} \operatorname{tr} \mathbf{H}\left(1-P_{2}-2 P_{3}\right),
\end{aligned}
$$

where the "indices of purity" $P_{1}, P_{2}, P_{3}$, are defined as

$$
\begin{array}{r}
P_{1} \equiv \frac{\lambda_{0}-\lambda_{1}}{\operatorname{tr} \mathbf{H}}, P_{2} \equiv \frac{\left(\lambda_{0}+\lambda_{1}\right)-\left(\lambda_{2}+\lambda_{3}\right)}{\operatorname{tr} \mathbf{H} \quad} \\
P_{3} \equiv \frac{\lambda_{2}-\lambda_{3}}{\operatorname{tr} \mathbf{H}} .
\end{array}
$$

We see that they are expressed in a similar way to the degree of polarization. In fact:

- $P_{1}$ is a non-dimensional relative measure of the difference between the weights of the two more significant pure components of the system;

- $P_{2}$ is a non-dimensional relative measure of the difference between the combined weight of the two more significant pure components and the combined weight of the two less significant pure components of the system;

- $P_{3}$ is a non-dimensional relative measure of the difference between the weights of the two less significant pure components.

We see that the indices of purity can be interpreted as probabilistic relative measures, which provide complete information about the relative amounts of the "equivalent pure components" of the target. 
From the above equations, the following quadratic relation between $P_{(4)}$ and the three indices of purity $P_{1}, P_{2}$, $P_{3}$ is obtained

$$
P_{(4)}^{2}=\frac{1}{3}\left(2 P_{1}^{2}+P_{2}^{2}+2 P_{3}^{2}\right) .
$$

Another interesting expression of $P_{(4)}$ as a symmetric quadratic mean of all the relative differences between pairs of eigenvalues is given by

$$
P_{(4)}^{2}=\frac{1}{3} \sum_{\substack{i, j=0 \\ i<j}}^{3} p_{i j}^{2}, \quad p_{i j} \equiv \frac{\lambda_{i}-\lambda_{j}}{\operatorname{tr} \mathbf{H}} .
$$

Pure systems are characterized by $P_{(4)}=P_{1}=P_{2}=$ $1, P_{3}=0$. Moreover, the values $P_{(4)}=P_{1}=P_{2}=P_{3}=0$ correspond to certain equiprobable mixtures of four (or more) incoherent elements, resulting in a Mueller matrix $\mathbf{O}$ whose elements are zero except for $o_{00}$.

The degree of purity provides a global measure of the purity of the system, whereas a detailed analysis requires a consideration of the three indices of purity.

By applying the starting conditions for the eigenvalues $0 \leqslant \lambda_{3} \leqslant \lambda_{2} \leqslant \lambda_{1} \leqslant \lambda_{0}$, we find that the indices of purity are restricted by the following conditions

$$
P_{1} \geqslant 0, P_{3} \geqslant 0, P_{1}+P_{3} \leqslant P_{2}, P_{3} \leqslant \frac{1}{2}\left(1-P_{2}\right) .
$$

Some useful inequalities derived from these conditions, but less restrictive and hence insufficient for re-obtaining the conditions given by equation (211) are the following

$0 \leqslant P_{1} \leqslant P_{2} \leqslant 1,0 \leqslant P_{3} \leqslant P_{2} \leqslant 1,0 \leqslant P_{3} \leqslant \frac{1}{3}\left(1-P_{1}\right)$.

Figure 11 shows the feasible region of the purity indices in the purity space. The restriction to the plane $P_{3}=0$ reproduces the feasible region for the indices of purity $P_{1}, P_{2}$ corresponding to $3 \times 3$ coherency matrices, and the feasible region for the degree of polarization of $2 \times 2$ coherency matrices corresponds to the segment $P_{2}=1,0 \leqslant P_{1} \leqslant 1$.

Figure 11 summarizes the different physically realizable possibilities in terms of the values of the indices of purity. Nevertheless, it is worth considering some particular cases in order to understand the physical meaning of $P_{i}$, as well as to reproduce the feasible regions for 3D and $2 \mathrm{D}$ representations of the indices of purity.

Given a fixed value of $P_{1}$, the maximum of $P_{3}$ is $P_{3}=$ $\frac{1}{3}\left(1-P_{1}\right)$ which is reached when $P_{2}=\frac{1}{3}\left(1+2 P_{1}\right)$.

Given fixed values for $P_{1}$ and $P_{2}$, the maximum of $P_{3}$ must be analyzed through the two following cases

(i) $P_{2} \leqslant \frac{1}{3}\left(1+2 P_{1}\right) \Rightarrow P_{3} \leqslant P_{2}-P_{1}$

(ii) $P_{2} \geqslant \frac{1}{3}\left(1+2 P_{1}\right) \Rightarrow P_{3} \leqslant \frac{1}{2}\left(1-P_{2}\right)$.

Next we consider some interesting cases based on the values of the indices of purity.

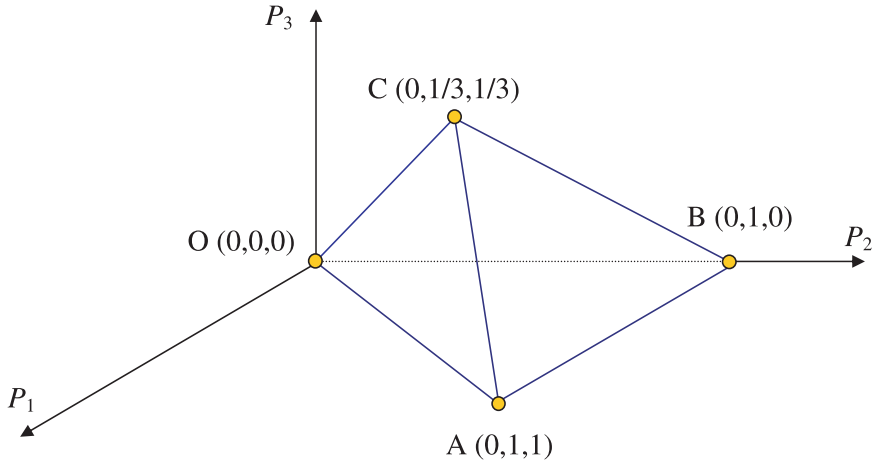

Fig. 11. (Color online) Feasible region for $P_{1}, P_{2}, P_{2}$ in the purity space.

(a) The face OBA corresponds to states with $P_{3}=$ 0 . Here we analyze the ranges $0<P_{1} \leqslant$ $P_{2} \leqslant 1 \quad\left(0<P_{(4)} \leqslant 1\right)$. The system is composed of two or four pure elements (if there are four, the two less significant have equal cross sections $\left.\lambda_{2}=\lambda_{3}\right)$. The feasible region is determined by the points placed on the triangle OBA (vertex $\mathrm{O}$ excluded). This case is mathematically equivalent to that obtained for $3 \times 3$ coherency matrices (3D polarization states).

$P_{3}=0, P_{1}=0,0<P_{2}<1\left(0<P_{(4)}<1 / \sqrt{3}\right)$.

The system is composed of four pure elements. The two more significant have equal cross sections $\lambda_{0}=$ $\lambda_{1}$ and the two less significant have equal cross sections $\lambda_{2}=\lambda_{3}$. The feasible region is the edge $\mathrm{OB}$ (vertices $\mathrm{O}$ and $\mathrm{B}$ excluded). These states are mathematically equivalent to states of light with random ellipse and whose direction of propagation is not fixed.

(a.2) $P_{3}=0,0 \leqslant P_{1} \leqslant 1, P_{2}=1\left(1 / \sqrt{3} \leqslant P_{(4)} \leqslant 1\right)$. The system is composed of two pure elements. The feasible region is the edge $\mathrm{BA}$ and $P_{1}$ is mathematically equivalent to the $2 \mathrm{D}$ degree of polarization $P_{(2)}$.

(a.2.1) $P_{3}=0, P_{1}=P_{2}=1 \quad\left(P_{(4)}=1\right)$. Pure system (non-depolarizing deterministic system) characterized by a Mueller-Jones matrix. The vertex A represents this state.

(a.2.2) $P_{3}=0, P_{1}=0, P_{2}=1\left(P_{(4)}=1 / \sqrt{3}\right)$. The system is composed of two pure elements with equal cross sections. The point $\mathrm{B}$ represents this state and the case is mathematically equivalent to $2 \mathrm{D}$ unpolarized light.

(b) The face OBC corresponds to states with $P_{1}=0$. Here we consider the ranges $0<P_{2} \leqslant 1,0<$ $P_{3} \quad\left(0<P_{(4)} \leqslant 1 / \sqrt{3}\right)$. The system is composed of three or four pure elements. The two more significant have equal cross sections $\lambda_{0}=\lambda_{1}$ whereas the two less significant have different cross sections $\lambda_{2} \neq \lambda_{3}$. The face OBC (excluding the segment $\mathrm{OB}$ ) determines the feasible region. These states are exclusive of the $4 \mathrm{D}$ representation 
because the reproduction of $3 \mathrm{D}$ states (and, hence, possible $2 \mathrm{D}$ states) requires the equality $\lambda_{2}=\lambda_{3}$.

(b.1) $\quad P_{1}=0,0<P_{2}=P_{3} \quad\left(P_{(4)} \leqslant 1 / \sqrt{3}\right)$. The system is composed of three pure elements with equal cross sections $\left(0<\lambda_{0}=\lambda_{1}=\lambda_{2}, \lambda_{3}=0\right)$ and is represented by the edge OC (vertex $\mathrm{O}$ excluded).

(b.1.1) $P_{1}=0, P_{2}=P_{3}=\frac{1}{3} \quad\left(P_{(4)}=1 / \sqrt{3}\right)$. The system is composed of three pure elements with equal cross sections. The vertex $\mathrm{C}$ represents this state.

(c) The face CBA (excluded the edge BA) corresponds to states with $\frac{1}{3} \leqslant P_{2}<1, P_{3}=\frac{1}{2}\left(1-P_{2}\right), P_{1} \leqslant$ $P_{2} \quad\left(1 / \sqrt{3} \leqslant P_{(4)}<1\right)$. The system is composed of three or four elements. If there are four, the two less significant have different cross sections. These states are exclusive of the $4 \mathrm{D}$ representation.

(d) The face OCA (vertex O excluded) corresponds to states with $P_{2}=P_{1}+P_{3}, P_{1}<P_{2}\left(0 \leqslant P_{(4)} \leqslant 1\right)$. The system is composed of four pure elements. The three more significant have equal cross-sections $\lambda_{1}=\lambda_{2}=\lambda_{3}$ and different from the least significant. These states are exclusive of the $4 \mathrm{D}$ representation. The edge $\mathrm{CA}$ corresponds to $P_{2}=$ $\frac{1}{3}\left(1+2 P_{1}\right)$.

(e) $\stackrel{P}{2}_{2}=0 \Rightarrow P_{1}=P_{2}=P_{3}=P_{(4)}=0$. This system, equivalent to an ideal depolarizer, is composed of four pure elements with equal cross sections and is represented by the vertex A. All the elements of the Mueller matrix are zero except for $m_{00}$.

\subsection{Polarization entropy}

Experimental and industrial polarimetry involve the measurement of up to 16 physical magnitudes which are constrained by several complicated non-linear relations. Thus considerable efforts have been made to take advantage of the polarimetric measurements. Polarization entropy $S$ [57] is a concept related with non-purity of the material samples. The measurement of $S$ is useful for several purposes and is commonly used in problems concerning light scattering, SAR polarimetry and, in general, situations where depolarization is a relevant subject. This nondimensional parameter, which is directly related with the degree of purity of the material sample, is defined as

$$
S(\mathbf{H})=-\operatorname{tr}(\hat{\mathbf{H}} \ln \hat{\mathbf{H}})=-\sum_{i=0}^{3}\left(\hat{\lambda}_{i} \ln \hat{\lambda}_{i}\right)
$$

where $\hat{\mathbf{H}}=\mathbf{H} / \operatorname{tr} \mathbf{H}$ is the density matrix and $\hat{\lambda}_{0}, \hat{\lambda}_{1}, \hat{\lambda}_{2}, \hat{\lambda}_{3}$ are the eigenvalues of $\hat{\mathbf{H}}$.

As we have seen, the indices of purity provide complete information about the depolarization properties and about the polarimetric purity of material samples, in such a manner that they cover a scope of information wider than $S$ and $P_{(4)}$. In fact, $S$ and $P_{(4)}$ can be directly obtained from the indices of purity.

The study of the relation between the relevant parameters $P_{(4)}$ and $S$ leads to the result that all scattering media must satisfy some universal constraints [193,201]. These constraints apply to both classical and quantum scattering processes and have important applications in fields such as quantum communication where depolarization is related with decoherence.

Moreover, given the practical importance of having suitable techniques for improving the analysis of polarimetric images, as we have indicated previously, some recent works $[108,115]$ have dealt with the concept of Shannon entropy and Kullback relative entropy for optical waves, and their relations with the $n$-dimensional degrees of polarization. We see that these concepts can be applied not only to $n$-dimensional partially polarized light, but also to the analysis of the polarimetric purity of material systems.

Following the idea introduced in the section devoted to the entropy of $3 \times 3$ coherency matrices, it is possible to extend to $4 \times 4$ coherency matrices the concept of partial entropies by defining the following quantities: $d i$ rectional entropy $S_{(2)}\left(P_{2}\right)$; polarization entropy $S_{(2)}\left(P_{1}\right)$, and residual entropy $S_{(2)}\left(P_{3}\right)$. The latter is exclusive of $n \times n$ coherency matrices with $n \geqslant 4$.

\subsection{Unified polarization algebra}

The intimate relation between $n \times n$ coherency matrices and the Lie groups $\mathrm{SU}(n)$ has been pointed out and studied by Cloude [161-163] who has shown that this is a suitable and fruitful framework for polarization algebra.

The polarization states are characterized by means of their respective coherency matrices, which contain all the physical measurable information [202].

In fact, throughout the sections devoted to the mathematical representation of the polarimetric properties of light and optical systems, we have seen that there exists a strong and clear symmetry in the description of these properties. The expansion of the $n \times n$ coherency matrix in a basis of $n$ trace orthogonal Hermitian matrices leads to the corresponding $n \mathrm{D}$ Stokes parameters. The overall polarimetric purity is defined by means of the degree of purity $P_{(n)}$, whereas the detailed information about purity is given by the corresponding $(n-1)$ set of indices of purity. The relation between the norms associated with the coherency matrix provides an appropriate purity criterion.

Thus, if $\boldsymbol{\Omega}$ is a $n \times n$ coherency matrix and $\boldsymbol{\Theta}_{i j}(i, j=0, n-1)$ constitute a basis of Hermitian trace orthogonal $n \times n$ matrices composed of the $n-1$ traceless generators of the $\mathrm{SU}(n)$ group plus the identity matrix, all of them satisfying $\boldsymbol{\Theta}_{i j}^{2}=\mathbf{1}$ and $\operatorname{tr}\left(\boldsymbol{\Theta}_{i j} \boldsymbol{\Theta}_{k l}\right)=n \delta_{i k} \delta_{j l}$, the real coefficients $c_{i j}=\operatorname{tr}\left(\boldsymbol{\Theta}_{i j} \boldsymbol{\Omega}\right)$ of the expansion

$$
\boldsymbol{\Omega}=\frac{1}{n} \sum_{i, j=0}^{n-1} c_{i j} \mathbf{\Theta}_{i j}
$$

are the measurable quantities ( $n \mathrm{D}$ Stokes parameters) characterizing completely the polarimetric properties of the system. 
The following relations are satisfied

$\|\boldsymbol{\Omega}\|_{2}^{2}=\frac{1}{n}\|\mathbf{C}\|_{2}^{2}, \quad\|\boldsymbol{\Omega}\|_{0}=c_{00}, \quad \frac{1}{n}\|\boldsymbol{\Omega}\|_{0}^{2} \leqslant\|\boldsymbol{\Omega}\|_{2}^{2} \leqslant\|\boldsymbol{\Omega}\|_{0}^{2}$,

where \|\|$_{2}$ stands for Euclidean norm; the norm \|\|$_{0}$ is defined as $\|\boldsymbol{\Omega}\|_{0} \equiv \operatorname{tr} \boldsymbol{\Omega}=\|\sqrt{\boldsymbol{\Omega}}\|_{2}^{2}$, and $\mathbf{C}$ is the matrix whose elements are $c_{i j}$.

Therefore we can state the following purity criterion: given a coherency matrix $\boldsymbol{\Omega},\|\boldsymbol{\Omega}\|_{2}^{2}=\|\boldsymbol{\Omega}\|_{0}^{2}$ is a necessary and sufficient condition for $\boldsymbol{\Omega}$ to represent a pure system.

The degree of purity is defined as the following invariant non-dimensional quantity

$$
P_{(n)}=\left|\left[\frac{1}{n-1}\left(\frac{n\|\boldsymbol{\Omega}\|_{2}^{2}}{\|\boldsymbol{\Omega}\|_{0}^{2}}-1\right)\right]^{1 / 2}\right|
$$

which gives a global measure of the purity of the system, so that $0 \leqslant P_{(n)} \leqslant 1$. The minimum value of the degree of purity $P_{(n)}=0$ corresponds to a fully random system, whereas the maximum value $P_{(n)}=1$ corresponds to a pure system.

The degree of purity can also be expressed as

$$
P_{(n)}^{2}=\frac{1}{n-1} \sum_{\substack{i, j=0 \\ i<j}}^{n} p_{i j}^{2}, \quad p_{i j} \equiv \frac{\lambda_{i}-\lambda_{j}}{\operatorname{tr} \mathbf{H}} .
$$

This has a particular relevance because it provides information similar to the entropy, but in a simpler and meaningful way. Through the indices of purity, the invariant information is structured in an optimum manner, so that these results can be useful in other fields of Physics. For example, in the same way that the Bloch equations lead to a set of parameters with the same mathematical properties as the Stokes parameters [46, 203-205], the degree of purity and the indices of purity can be applied to $n$-dimensional density matrices characteristics of $n$-level systems [180].

In general, the above-mentioned invariant quantities can be applied to any problem susceptible to modeling by means of $n \times n$ positive semidefinite Hermitian matrices.

Therefore, $n-1$ indices of purity $P_{i}$ are necessary for the complete characterization of the polarimetric purity of the system.

Concerning the different decompositions of the coherency matrix $\boldsymbol{\Omega}$, it is straightforward to generalize the spectral, trivial, and arbitrary decompositions. Unlike the spectral decomposition, which is widely known and used, the two others are generally not known and we emphasize the high potential of their applications in polarimetry and in other fields.

\subsection{Macroscopic polarimetric behaviors of material media.}

In previous sections, we have analyzed the properties of pure retarders and pure diattenuators. Now we consider the overall properties of systems composed of an incoherent mixture of pure elements (incoherent parallel combination).

An alternative view of the Stokes-Mueller model is based on a consideration of the spectral Stokes vector $\mathbf{s}(\nu)$ (related with the corresponding spectral coherency matrix). Under the assumption of cross-spectral purity $[7,118], \mathbf{s}(\nu)$ can be expressed as

$$
\mathbf{s}(\nu)=g(\nu) \overline{\mathbf{s}},
$$

where $g(\nu)$ is the normalized spectral distribution of the light beam and $\overline{\mathbf{s}}$ is a Stokes vector independent of frequency. The medium is represented by its corresponding "spectral Mueller-Jones matrix" $\mathbf{N}(\nu)$ so that the spectral stokes vector of the outgoing light is given by

$$
\mathbf{s}^{\prime}(\nu)=\mathbf{N}(\nu) \mathbf{s}(\nu),
$$

and, integrating over all frequencies,

$$
\overline{\mathbf{s}^{\prime}}=\mathbf{M} \overline{\mathbf{s}},
$$

where $\overline{\mathbf{s}}$ is the resulting integrated Stokes vector and $\mathbf{M}$ is the integrated Mueller matrix defined by Dlugnikov [118]

$$
\overline{\mathbf{s}^{\prime}} \equiv \int_{0}^{\infty} \mathbf{s}^{\prime}(\nu) d \nu, \quad \mathbf{M} \equiv \int_{0}^{\infty} g(\nu) \mathbf{N}(\nu) d \nu .
$$

By means of this formulation, some authors have studied the effects of dispersive media on the degree of polarization of the transmitted light $[117,119,206]$.

Let us now analyze the polarizance/diattenuation $[131,149,200,207]$ properties of $\mathbf{M}$. All the information about diattenuation is included in its first row, whereas all information about polarizance is included in its first column. Taking into account the reciprocity properties of Mueller matrices, we will at times refer to the polarizance vector as the "forward polarizance vector" $\mathbf{\Upsilon}_{\mathbf{f}}$ and to the diattenuation vector as the "reverse polarizance vector" $\Upsilon_{\mathbf{r}}$

$\mathbf{\Upsilon}_{f} \equiv \frac{1}{m_{00}}\left(m_{10}, m_{20}, m_{30}\right)^{T}, \quad \mathbf{\Upsilon}_{r} \equiv \frac{1}{m_{00}}\left(m_{01}, m_{02}, m_{03}\right)^{T}$,

whose modules are the "forward polarizance" (polarizance) $\Upsilon_{f}$ and the "reverse polarizance" (diattenuation) $\Upsilon_{r}$. Thus, in general, we will use the terms diattenuator and polarizer as synonymous.

The polarizances are restricted to the following values

$$
0 \leqslant \Upsilon_{f} \leqslant 1, \quad 0 \leqslant \Upsilon_{r} \leqslant 1
$$

Following the notation suggested by Xing [152] and Lu and Chipman [149,208], a Mueller matrix can be written as

$$
\mathbf{M}=m_{00}\left(\begin{array}{ll}
1 & \mathbf{\Upsilon}_{r}^{T} \\
\boldsymbol{\Upsilon}_{f} & \overline{\mathbf{M}}
\end{array}\right) ; \quad \overline{\mathbf{M}} \equiv \frac{1}{m_{00}}\left(\begin{array}{lll}
m_{11} & m_{12} & m_{13} \\
m_{21} & m_{22} & m_{23} \\
m_{31} & m_{32} & m_{33}
\end{array}\right)
$$


Taking into account the expressions of the extreme values of the transmittance

$$
m_{00}\left(1+\Upsilon_{f}\right) \leqslant 1, \quad m_{00}\left(1+\Upsilon_{r}\right) \leqslant 1,
$$

it is clear that $\mathbf{\Upsilon}_{r}, \mathbf{\Upsilon}_{f}$ provide all the information about forward and reverse diattenuation respectively.

In general, depolarization and diattenuation appear in a combined form. Pure systems are characterized by their respective Mueller-Jones matrices and do not produce depolarization effects on incoming totally polarized light. Mueller-Jones matrices are Mueller matrices that satisfy $P_{(4)}(\mathbf{M})=1$ and, consequently, have the property $\Upsilon_{f}=\Upsilon_{r}$.

Incoherent mixtures (parallel combinations) of pure systems produce depolarizing effects, which can appear combined with retardation and polarization properties. It is important to observe that $\Upsilon_{f}=\Upsilon_{r}$ is a necessary but not a sufficient condition for a Mueller matrix to be a MuellerJones matrix. Moreover, $\Upsilon_{f} \neq \Upsilon_{r}$ implies that the system is non-pure, while $\Upsilon_{f}=\Upsilon_{r}=1$ implies total purity.

It is interesting to consider some cases that are representative of different physical behaviors of material media.

(a) $\Upsilon_{f}>0, \Upsilon_{r}=0$. The system exhibits forward polarizance, zero reverse polarizance and depolarization. An example of this is a system composed of an ideal depolarizer followed by a total polarizer.

$$
\mathbf{N}_{D}(\beta, 0,1,1)\left(\begin{array}{llll}
1 & 0 & 0 & 0 \\
0 & 0 & 0 & 0 \\
0 & 0 & 0 & 0 \\
0 & 0 & 0 & 0
\end{array}\right)=\left(\begin{array}{llll}
n_{00} & 0 & 0 & 0 \\
n_{10} & 0 & 0 & 0 \\
n_{20} & 0 & 0 & 0 \\
n_{30} & 0 & 0 & 0
\end{array}\right) \text {. }
$$

(b) $\Upsilon_{r}>0, \Upsilon_{f}=0$. The system exhibits reverse polarizance, zero forward polarizance and depolarization. An example is a system composed of a total polarizer followed by an ideal depolarizer. The resulting matrix has the form of the transposed matrix of the above matrix.

(c) $\Upsilon_{f}=1,0<\Upsilon_{r}<1$. The system exhibits maximum forward polarizance, reverse polarizance and depolarization. An example is a serial combination constituted by a partial polarizer, an ideal depolarizer and a total polarizer, in this order. Although the system produce totally polarized light for all incoming states, the "internal" depolarizing effect affects the corresponding Mueller matrix, so that this non-pure system cannot be represented by a Jones-Mueller matrix.

(d) $0<\Upsilon_{f}<1, \Upsilon_{r}=1$. The system exhibits maximum reverse polarizance, forward polarizance and depolarization. An example is a serial combination constituted by a total polarizer, an ideal depolarizer and a partial polarizer, in that order. Obviously, this case is the "reciprocal" of the previous one.

(e) $0<\Upsilon_{f}<1,0<\Upsilon_{r}<1, \Upsilon_{f} \neq \Upsilon_{r}$. The system exhibits forward polarizance, reverse polarizance and depolarization. An example is a serial combination constituted by an ideal polarizer placed between two partial polarizers. (f) $0<\Upsilon_{f}<1, \Upsilon_{f}=\Upsilon_{r}$. The system exhibits equal polarizances. This condition does not determine if the system exhibits depolarization or not. The global purity of the system is given by $P_{(4)}$.

(g) $\Upsilon_{f}=\Upsilon_{r}=1$. The system exhibits maximum forward polarizance and maximum reverse polarizance and is equivalent to a serial combination of pure or non-pure elements placed between two total polarizers. It is important to note that the system behaves as pure regardless of the possible existence of depolarizers placed between the total polarizers. An interesting example is an ideal depolarizer placed between two total polarizers

$$
\mathbf{M}=\mathbf{N}^{\prime}(\beta, 1,1)\left(\begin{array}{llll}
1 & 0 & 0 & 0 \\
0 & 0 & 0 & 0 \\
0 & 0 & 0 & 0 \\
0 & 0 & 0 & 0
\end{array}\right) \mathbf{N}(\theta, 1,1) .
$$

It is easy to show that in this case $P_{(4)}=1$, despite the effect of the intermediate depolarizer, which affects only to the transparency of the complete system represented by $\mathbf{M}$.

\subsection{The generalized polar decomposition}

Some models for the serial decomposition of Stokes matrices have been introduced by Xing [152] and Sridhar and Simon [160] on the basis of the Stokes criterion. As van der Mee has pointed out [167], these decompositions are not totally general. Moreover, $\mathrm{Lu}$ and Chipman have emphasized the necessity of serial decompositions where the basic physical behaviors (diattenuation/polarization, retardation and depolarization) appear explicitly in the different components of the equivalent serial system and have presented an algorithm to decompose a Mueller matrix into a serial combination of a diattenuator, a retarder and a partial polarizer-depolarizer [149]. This "generalized polar decomposition" of a Mueller matrix is currently used for many purposes concerning the physical interpretation of experimental measures as well as for studying physical models of several kinds of material targets [209-211].

\subsubsection{The Lu-Chipman decomposition}

In this sub-section we summarize the main results of the cited work of Lu and Chipman [149].

Taking into account the expression of a Mueller matrix $\mathbf{M}$ given by equation (224) these authors have shown that $\mathbf{M}$ can be written as [149]

$$
\begin{aligned}
\mathbf{M} & =m_{00}\left(\begin{array}{cc}
1 & \mathbf{\Upsilon}_{r}^{T} \\
\boldsymbol{\Upsilon}_{f} & \overline{\mathbf{M}}
\end{array}\right)=m_{00} \mathbf{C N}_{R} \mathbf{A} \\
\mathbf{C} & \equiv\left(\begin{array}{cc}
1 & \mathbf{0}^{T} \\
\boldsymbol{\Upsilon}_{\Delta} & \overline{\mathbf{C}}
\end{array}\right) \\
\mathbf{N}_{R} & \equiv\left(\begin{array}{cc}
1 & \mathbf{0}^{T} \\
\mathbf{0} & \overline{\mathbf{N}}_{R}
\end{array}\right), \mathbf{A} \equiv\left(\begin{array}{cc}
1 & \boldsymbol{\Upsilon}_{r}^{\mathrm{T}} \\
\boldsymbol{\Upsilon}_{r} & \overline{\mathbf{N}}_{D}\left(\boldsymbol{\Upsilon}_{r}\right)
\end{array}\right)
\end{aligned}
$$


where $\mathbf{0}^{T} \equiv(0,0,0)$ and:

- the matrix $\mathbf{A}$ is proportional to a pure diattenuator;

- the matrix $\mathbf{N}_{R}$ represents a pure retarder;

- the matrix $\mathbf{C}$ is proportional to a polarizer-depolarizer with zero diattenuation. This class of system exhibits simultaneously both depolarizing and polarizing properties.

As indicated in equation (226), the matrix A can be calculated from the diattenuation vector $\boldsymbol{\Upsilon}_{r}$ of $\mathbf{M}$. Moreover,

$$
\Upsilon_{\Delta}=\frac{\Upsilon_{f}-\overline{\mathbf{M}} \Upsilon_{r}}{1-\Upsilon_{r}^{2}}
$$

For the calculation of $\overline{\mathbf{C}}$ and $\overline{\mathbf{N}}_{R}$ the cases of $\mathbf{M}$ nonsingular and $\mathbf{M}$ singular must be distinguished

\section{(a) M non-singular}

In this case $\mathbf{A}$ is non-singular, so that it is possible to define the matrix $\mathbf{M}^{\prime}=\mathbf{M} \mathbf{A}^{-1}$. This matrix has no diattenuation and can be written as

$$
\mathbf{M}^{\prime}=\frac{1}{m_{00}} \mathbf{C N}_{R}=\frac{1}{m_{00}}\left(\begin{array}{ll}
1 & \mathbf{0}^{T} \\
\mathbf{\Upsilon}_{\Delta} & \overline{\mathbf{M}}^{\prime}
\end{array}\right),
$$

and the calculation of the matrices $\overline{\mathbf{C}}$ and $\overline{\mathbf{N}}_{R}$ can be performed through singular value decomposition of the matrix $\overline{\mathbf{M}}^{\prime} \equiv \overline{\mathbf{C}} \overline{\mathbf{N}}_{R}$

$$
\overline{\mathbf{M}}^{\prime}=\overline{\mathbf{U}}\left[\mathbf{D}\left(d_{1}, d_{2}, d_{3}\right)\right] \overline{\mathbf{V}}, \quad d_{1} \geqslant d_{2} \geqslant d_{3} \geqslant 0,
$$

where $\overline{\mathbf{U}}, \overline{\mathbf{V}}$ are $3 \times 3$ orthogonal matrices and $d_{i}$ are the eigenvalues of the positive semi-definite symmetric matrix $\overline{\mathbf{M}}^{\prime} \overline{\mathbf{M}}^{\prime} \mathbf{T}$.

Finally the matrices $\overline{\mathbf{C}}$ and $\overline{\mathbf{N}}_{R}$ are given by

$$
\begin{aligned}
\overline{\mathbf{C}} & =\left(\frac{\operatorname{det} \overline{\mathbf{U}} \overline{\mathbf{V}}}{|\operatorname{det} \overline{\mathbf{U}} \overline{\mathbf{V}}|}\right) \overline{\mathbf{U}} \mathbf{D}\left(d_{1}, d_{2}, d_{3}\right) \overline{\mathbf{U}}^{T}, \\
\overline{\mathbf{N}}_{R} & =\left(\frac{\operatorname{det} \overline{\mathbf{U}} \overline{\mathbf{V}}}{|\operatorname{det} \overline{\mathbf{U}} \overline{\mathbf{V}}|}\right) \overline{\mathbf{U}} \overline{\mathbf{V}} .
\end{aligned}
$$

We observe that $\overline{\mathbf{C}}$ is a positive semidefinite symmetric matrix and $\overline{\mathbf{N}}_{R}$ is a proper rotation matrix $\left(\operatorname{det} \overline{\mathbf{N}}_{R}=1\right)$. Moreover, $\overline{\mathbf{C}}, \overline{\mathbf{N}}_{R}$ are the symmetric and orthogonal components corresponding to the polar decomposition of $\overline{\mathbf{M}}^{\prime}$. This is the origin of the name "generalized polar decomposition" given by Lu and Chipman to the decomposition (226) despite the fact that, as we will see in the next section, the direct application of the polar decomposition to $\mathbf{M}$ results in an unphysical orthogonal component.

It should be noted that the matrices $\mathbf{A}, \mathbf{C}$ do not satisfy the transmittance conditions. A physical decomposition must be expressed as

$$
\mathbf{M}=c \mathbf{C} b \mathbf{N}_{R} a \mathbf{A} ; \quad a, b, c \geqslant 0, \quad a b c=m_{00},
$$

where $b \leqslant 1$ and $a, c$ must be small enough to satisfy

$$
a\left(1+\Upsilon_{r}\right) \leqslant 1, \quad c\left(1+\Upsilon_{\Delta}\right) \leqslant 1
$$

Thus, the following condition is required for a physically realizable decomposition

$$
\left(1+\Upsilon_{r}\right)\left(1+\Upsilon_{\Delta}\right) \leqslant \frac{1}{m_{00}}
$$

It is clear that the components of the generalized polar decomposition are Stokes matrices. Nevertheless, the conventional form of the generalized polar decomposition assigns the coefficient $m_{00}$ to the equivalent diattenuator so that the resulting polarizer-depolarizer component does not satisfy the reverse transmittance condition. Thus, further work is required in clarifying the conditions under which all the components of the generalized polar decomposition are Mueller matrices, i.e. they satisfy the transmittance conditions as well as the covariance conditions.

Provided equation (233) is satisfied, the Mueller matrix $\mathbf{M}$ can be decomposed as a product $\mathbf{M}=\mathbf{M}_{\Delta} \mathbf{N}_{R} \mathbf{N}_{D}$ of a pure diattenuator $\mathbf{N}_{D}$, a pure retarder $\mathbf{N}_{R}$ and a polarizer-depolarizer $\mathbf{M}_{\Delta}$.

\section{(b) M singular}

In this case, at least one of the following equalities is satisfied: $\Upsilon_{f}=1, \Upsilon_{r}=1$ and $\mathbf{M}$ can be decomposed as

$$
\begin{aligned}
\mathbf{M} & =m_{00}\left(\begin{array}{ll}
1 & \mathbf{\Upsilon}_{r}^{T} \\
\mathbf{\Upsilon}_{f} & \mathbf{\Upsilon}_{f} \otimes \mathbf{\Upsilon}_{r}^{T}
\end{array}\right)=m_{00} \mathbf{M}_{I} \mathbf{N}_{R} \mathbf{B} \\
\mathbf{M}_{I} & \equiv\left(\begin{array}{ll}
1 & \mathbf{0}^{T} \\
\mathbf{0} & \mathbf{\Upsilon}_{f} \mathbf{D}(1,1,1)
\end{array}\right), \mathbf{N}_{R} \equiv\left(\begin{array}{ll}
1 & \mathbf{0}^{T} \\
\mathbf{0} & \overline{\mathbf{N}}_{R}
\end{array}\right), \\
\mathbf{B} & \equiv\left(\begin{array}{ll}
1 & \mathbf{\Upsilon}_{r}^{\mathrm{T}} \\
\mathbf{\Upsilon}_{r} & \mathbf{\Upsilon}_{r} \otimes \mathbf{\Upsilon}_{r}^{\mathrm{T}}
\end{array}\right),
\end{aligned}
$$

where $m_{00} \mathbf{B}$ is the Mueller matrix of a homogeneous pure diattenuator and $\mathbf{M}_{I}$ represents a "pure depolarizer".

\subsubsection{The forward and reverse generalized polar decompositions}

Let us now consider now the possible different decompositions obtainable by changing the order of the three components. Lu and Chipman [149], Morio and Goudail [212] and Ossikovski, De Martino and Guyot [213] have studied the six possible decompositions as well as some examples where certain decompositions lead to unphysical components. According with the results of Morio and Goudail [212], the following decompositions, where the diattenuator is placed before the polarizer-depolarizer ("forward generalized polar decompositions"), always lead to components represented by Stokes matrices

$$
\mathbf{M}=\mathbf{M}_{\Delta 1} \mathbf{N}_{R 1} \mathbf{N}_{D 1}=\mathbf{M}_{\Delta 2} \mathbf{N}_{D 2} \mathbf{N}_{R 2}=\mathbf{N}_{R 5} \mathbf{M}_{\Delta 5} \mathbf{N}_{D 5}
$$

whereas the other possible decompositions, where the polarizer-depolarizer is placed before the diattenuator 
("reverse generalized polar decompositions"), are not always composed of Stokes matrices

$$
\mathbf{M}=\mathbf{N}_{R 3} \mathbf{N}_{D 3} \mathbf{M}_{\Delta 3}=\mathbf{N}_{D 4} \mathbf{N}_{R 4} \mathbf{M}_{\Delta 4}=\mathbf{N}_{D 6} \mathbf{M}_{\Delta 6} \mathbf{N}_{R 6} .
$$

Concerning these kinds of decompositions and their physical validity it is necessary to check the transmittance conditions for $\mathbf{M}$ and for the matrices of the different components. Some examples have been used in the literature where $m_{00}=1$ and $\Upsilon_{r} \neq 0$ (or $\Upsilon_{f} \neq 0$ ). These values never correspond to a Mueller matrix because at least one of the transmittance conditions is not satisfied.

Prior to analyze other possible decompositions of $\mathbf{M}$, let us consider the Stokes matrix of a "diattenuatordepolarizer"

$$
\mathbf{M}_{\Lambda} \equiv\left(\begin{array}{cc}
1 & \mathbf{\Upsilon}_{\Lambda}^{T} \\
\mathbf{0} & \overline{\mathbf{M}}_{\Lambda}
\end{array}\right)
$$

which exhibits zero polarizance, non-zero diattenuation and depolarization. We observe that to convert this Stokes matrix into a Mueller matrix, it must be multiplied by a coefficient $\alpha \leqslant 1 /\left(1+\Upsilon_{\Lambda}\right)$.

In a previous section we have shown that, given a Mueller matrix $\mathbf{M}, \mathbf{M}^{T}$ is also a Mueller matrix so that, given a Mueller matrix $\mathbf{M}$, always exist a Mueller matrix $\mathbf{M}^{\prime}=\mathbf{M}^{T}$ satisfying $\mathbf{M}=\mathbf{M}^{\prime T}$. Thus, by applying the Lu-Chipman decomposition to $\mathbf{M}^{T}$ we obtain

$$
\begin{aligned}
\mathbf{M} & \equiv \mathbf{M}^{\prime}=\left(\mathbf{M}^{\prime}{ }_{\Delta 1} \mathbf{N}_{R 1}^{\prime} \mathbf{N}_{D 1}^{\prime}\right)^{T} \\
& =\left(\mathbf{M}^{\prime}{ }_{\Delta 2} \mathbf{N}_{D 2}^{\prime} \mathbf{N}_{R 2}^{\prime}\right)^{T}=\left(\mathbf{N}_{R 5}^{\prime} \mathbf{M}_{\Delta 5}^{\prime} \mathbf{N}_{D 5}^{\prime}\right)^{T} \\
& =\mathbf{N}_{D 4} \mathbf{N}_{R 4} \mathbf{M}_{\Lambda 4}=\mathbf{N}_{R 3} \mathbf{N}_{D 3} \mathbf{M}_{\Lambda 3}=\mathbf{N}_{D 6} \mathbf{M}_{\Lambda 6} \mathbf{N}_{R 6} .
\end{aligned}
$$

We see that the operation of transposing does no affect the nature of the matrices of the pure components, whereas leads to replacing the polarizers-depolarizers by diattenuators-depolarizers.

Some relations between the pure components of each one of the three sets of decompositions can be derived from the polar decomposition of a pure Mueller matrix.

Experimental validation of one of the forms of the reverse generalized polar decomposition has been presented recently by Anastasiadou, Ben Hatit, Ossikovski, Guyot and De Martino [214].

\subsubsection{The general decomposition of a Mueller matrix}

Although the Lu-Chipman decomposition has been demonstrated to be a powerful tool for analyzing measured Mueller matrices, the depolarizer component combines polarizing and depolarizing properties. Thus, an additional effort to separate the retarding, polarizing and depolarizing properties is desirable.

The general characterization of the Mueller matrix introduced in Section 5.4 reveals a fundamental symmetry of Mueller matrices in the sense of any general property of a Muller matrix $\mathbf{M}$ can also be appropriately formulated for its transposed matrix $\mathbf{M}^{T}$. We now translate this basic symmetry to the Lu-Chipman decomposition in order to introduce a general decomposition of a Mueller matrix where the depolarizing properties are isolated from that related with retardation and polarization-diattenuation properties.

Let us consider a Mueller matrix $\mathbf{M}$ and apply one of the forward forms of the Lu Chipman decomposition, so that

$$
\mathbf{M}=\mathbf{M}_{\Delta 2} \mathbf{N}_{D 2} \mathbf{N}_{R 2} .
$$

Moreover $\mathbf{M}_{\Delta 2}$ can be expressed as the transposed matrix of the matrix of a diattenuator-depolarizer $\mathbf{M}_{\Lambda}$ with the form given by equation (237). Thus, the Lu-Chipman decomposition can be applied to $\mathbf{M}_{\Lambda}$ and obtain

$$
\mathbf{M}_{\Delta 2}=\left[\mathbf{M}_{\Lambda}\right]^{T}=\left[\mathbf{M}_{\Delta}^{\prime} \mathbf{N}_{D}^{\prime} \mathbf{N}_{R}^{\prime}\right]^{T}=\mathbf{N}_{R}^{\prime T} \mathbf{N}_{D}^{\prime T} \mathbf{M}_{\Delta}^{\prime T},
$$

where $\mathbf{N}_{R}^{\prime T}$ represents a pure retarder, $\mathbf{N}_{D}^{\prime T}$ represents a pure diattenuator and $\mathbf{M}_{\Delta}^{\prime T}$ represents a depolarizer with zero diattenuation and zero polarizance. Thus, $\mathbf{M}_{\Delta}^{\prime T}$ has the following form

$$
\mathbf{M}_{\Delta}^{\prime T} \equiv \mathbf{M}_{\Omega}=\left(\begin{array}{ll}
1 & \mathbf{0}^{T} \\
\mathbf{0} & \overline{\mathbf{M}}_{\Omega}
\end{array}\right),
$$

where $\overline{\mathbf{M}}_{\Omega}$ is a symmetric matrix which contain all information about the depolarizing properties of $\mathbf{M}$. Consequently, we can finally formulate the following general decomposition

$$
\mathbf{M}=\mathbf{N}_{R O} \mathbf{N}_{D O} \mathbf{M}_{\Omega} \mathbf{N}_{D I} \mathbf{N}_{R I}
$$

where $\mathbf{N}_{R I}, \mathbf{N}_{R O}$ represent respectively the "input and output equivalent pure retarders", $\mathbf{N}_{D I}, \mathbf{N}_{D O}$ represent respectively the "input and output equivalent pure diattenuators" and $\mathbf{M}_{\Omega}$ represents the "equivalent depolarizer".

Thus, if we assume that the components of the LuChipman decomposition are Mueller matrices (and not only Stokes matrices) and we take into account the polar decomposition of a pure system, we see that the previous result can be formulated by means of the following statement: Any system is polarimetrically equivalent to a serial combination constituted by a depolarizer placed between two pure components. Or, in other words: Any Mueller matrix can be expressed as the ordered product of the Mueller matrices of an "output pure system" a depolarizer and an "input pure system".

As we have seen in the section devoted to the polar decomposition of a pure system, always is possible to choose the order of the diattenuator and the retarder of the pure equivalent system and, consequently, any Mueller matrix can be decomposed in the following different alternative ways

$$
\begin{aligned}
& \mathbf{M}=\mathbf{N}_{R O} \mathbf{N}_{D O} \mathbf{M}_{\Omega} \mathbf{N}_{D I} \mathbf{N}_{R I} \\
& \mathbf{M}=\mathbf{N}_{D O}^{\prime} \mathbf{N}_{R O}^{\prime} \mathbf{M}_{\Omega} \mathbf{N}_{R I}^{\prime} \mathbf{N}_{D I}^{\prime} \\
& \mathbf{M}=\mathbf{N}_{D O}^{\prime} \mathbf{N}_{R O}^{\prime} \mathbf{M}_{\Omega} \mathbf{N}_{D I} \mathbf{N}_{R I} \\
& \mathbf{M}=\mathbf{N}_{R O} \mathbf{N}_{D O} \mathbf{M}_{\Omega} \mathbf{N}_{R I}^{\prime} \mathbf{N}_{D I}^{\prime}
\end{aligned}
$$




\subsection{The Kernel matrix of a Mueller matrix}

To complete the study of the general properties of Mueller matrices, in this section we consider the problem of identifying the Mueller component which is invariant from a physical point of view and, consequently, obtaining the corresponding set of physically invariant quantities involved in a Mueller matrix.

Although the polar decomposition is very useful for the study of pure systems, we will see that this kind of decomposition cannot be applied directly to the case of depolarizing systems because the orthogonal component is not, in general, a Mueller matrix. Nevertheless, some algebraic rearrangements allow us to construct a new model where the Mueller matrix is decomposed into Mueller matrices (and not only Stokes matrices) and the invariant physical quantities appear grouped in the Kernel-matrix whose definition will be introduced below.

Let us consider a Mueller matrix $\mathbf{M}$ and its singular value decomposition (denoted from now on as SVD)

$$
\mathbf{M}=\mathbf{X D Y}
$$

where $\mathbf{X}, \mathbf{Y}$ are $4 \times 4$ orthogonal matrices and $\mathbf{D}$ is the diagonal matrix whose elements are the non-negative, ordered, singular values

$$
d_{0} \geqslant d_{1} \geqslant d_{2} \geqslant d_{3}
$$

Except for the particular case of $\mathbf{M}$ is a diagonal matrix, the orthogonal matrices $\mathbf{X}, \mathbf{Y}$ are not Mueller matrices. In fact, they can be written as products of six elementary $4 \times 4$ rotation Givens matrices

$$
\begin{aligned}
& \mathbf{Q}_{1}\left(\varphi_{1}\right)=\left[\begin{array}{llll}
\cos \varphi_{1} & 0 & 0 & \sin \varphi_{1} \\
0 & 1 & 0 & 0 \\
0 & 0 & 1 & 0 \\
-\sin \varphi_{1} & 0 & 0 & \cos \varphi_{1}
\end{array}\right], \\
& \mathbf{Q}_{2}\left(\varphi_{2}\right)=\left[\begin{array}{llll}
\cos \varphi_{2} & 0 & \sin \varphi_{2} & 0 \\
0 & 1 & 0 & 0 \\
-\sin \varphi_{2} & 0 & \cos \varphi_{2} & 0 \\
0 & 0 & 0 & 1
\end{array}\right] \text {, } \\
& \mathbf{Q}_{3}\left(\varphi_{3}\right)=\left[\begin{array}{lllll}
\cos \varphi_{3} & \sin \varphi_{3} & 0 & 0 \\
-\sin \varphi_{3} & \cos \varphi_{3} & 0 & 0 \\
0 & 0 & 1 & 0 \\
0 & 0 & 0 & 1
\end{array}\right] \\
& \mathbf{G}_{1}\left(\alpha_{1}\right)=\left[\begin{array}{llll}
1 & 0 & 0 & 0 \\
0 & 1 & 0 & 0 \\
0 & 0 & \cos \alpha_{1} & \sin \alpha_{1} \\
0 & 0 & -\sin \alpha_{1} & \cos \alpha_{1}
\end{array}\right], \\
& \mathbf{G}_{2}\left(\alpha_{2}\right)=\left[\begin{array}{llll}
1 & 0 & 0 & 0 \\
0 & \cos \alpha_{2} & 0 & \sin \alpha_{2} \\
0 & 0 & 1 & 0 \\
0 & -\sin \alpha_{2} & 0 & \cos \alpha_{2}
\end{array}\right],
\end{aligned}
$$

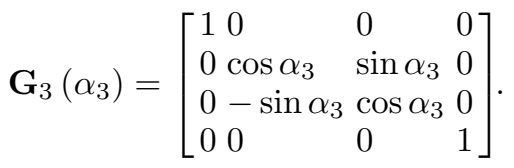

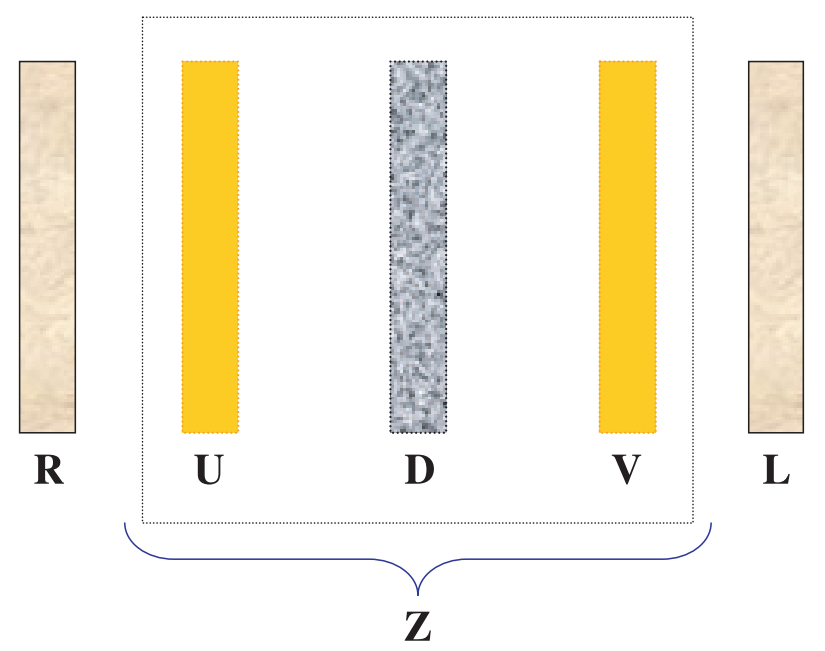

Fig. 12. (Color online) The kernel matrix of a Mueller matrix.

It should be noted that the order of the factors can be chosen arbitrarily. Then $\mathbf{X}, \mathbf{Y}$ can be written as

$$
\begin{aligned}
& \mathbf{X}=\mathbf{G}_{1}\left(\alpha_{1}\right) \mathbf{G}_{2}\left(\alpha_{2}\right) \mathbf{G}_{3}\left(\alpha_{3}\right) \mathbf{Q}_{1}\left(\varphi_{1}\right) \mathbf{Q}_{2}\left(\varphi_{2}\right) \mathbf{Q}_{3}\left(\varphi_{3}\right), \\
& \mathbf{Y}=\mathbf{Q}_{3}^{T}\left(\varphi_{6}\right) \mathbf{Q}_{2}^{T}\left(\varphi_{5}\right) \mathbf{Q}_{1}^{T}\left(\varphi_{4}\right) \mathbf{G}_{3}^{T}\left(\alpha_{6}\right) \mathbf{G}_{2}^{T}\left(\alpha_{5}\right) \mathbf{G}_{1}^{T}\left(\alpha_{4}\right) \\
& \text { or } \quad \mathbf{X}=\mathbf{R U}, \quad \mathbf{Y}=\mathbf{V L},
\end{aligned}
$$

where

$$
\begin{aligned}
\mathbf{R} & \equiv \mathbf{G}_{1}\left(\alpha_{1}\right) \mathbf{G}_{2}\left(\alpha_{2}\right) \mathbf{G}_{3}\left(\alpha_{3}\right) \\
\mathbf{U} & \equiv \mathbf{Q}_{1}\left(\varphi_{1}\right) \mathbf{Q}_{2}\left(\varphi_{2}\right) \mathbf{Q}_{3}\left(\varphi_{3}\right) \\
\mathbf{V} & \equiv \mathbf{Q}_{3}^{T}\left(\varphi_{6}\right) \mathbf{Q}_{2}^{T}\left(\varphi_{5}\right) \mathbf{Q}_{1}^{T}\left(\varphi_{4}\right) \\
\mathbf{L} & \equiv \mathbf{G}_{3}^{T}\left(\alpha_{6}\right) \mathbf{G}_{2}^{T}\left(\alpha_{5}\right) \mathbf{G}_{1}^{T}\left(\alpha_{4}\right)
\end{aligned}
$$

The matrices $\mathbf{R}, \mathbf{L}$ are Mueller matrices that represent pure retarders, whereas the matrices $\mathbf{U}, \mathbf{V}$ are neither Mueller matrices nor Stokes matrices. It is easy to see that they do not satisfy some of the conditions to be Mueller matrices. For example $\mathbf{Q}_{1}$ applied to the Stokes vector $(1,1,0,0)^{T}$ is not a Stokes vector.

The polar decompositions of $\mathbf{M}$ can be written as

$\mathbf{M}=\mathbf{K W} ; \mathbf{K} \equiv\left(\mathbf{M M}^{T}\right)^{1 / 2}=\mathbf{R U D U} \mathbf{U}^{T} \mathbf{R}^{T}, \mathbf{W} \equiv \mathbf{R U V L}$
$\mathbf{M}=\mathbf{W}^{\prime} \mathbf{K}^{\prime} ; \mathbf{W}^{\prime} \equiv \mathbf{R U V L}, \mathbf{K}^{\prime} \equiv\left(\mathbf{M}^{T} \mathbf{M}\right)^{1 / 2}=\mathbf{L}^{T} \mathbf{V}^{T} \mathbf{D V L}$

where the symmetric components $\mathbf{K}, \mathbf{K}^{\prime}$ are Mueller matrices whereas the orthogonal components $\mathbf{W}, \mathbf{W}^{\prime}$ are not Mueller matrices.

Nevertheless, it is possible to arrange the products so that the Mueller matrix can be written as (Fig. 12)

$$
\mathbf{M}=\mathbf{R Z L}
$$

where

$$
\mathbf{Z} \equiv \mathbf{U D V}
$$


We observe that this "kernel matrix" $\mathbf{Z} \equiv \mathbf{R}^{T} \mathbf{M} \mathbf{L}^{T}$ is a product of Mueller matrices and, hence, is a Mueller matrix. Thus, all the three components of the decomposition given by equation (251) are Mueller matrices (i.e. satisfy the transmittance conditions as well as the covariance conditions).

Moreover, the input and output retarders $\mathbf{L}, \mathbf{R}$, correspond to that obtained in the general decomposition given by equation (242), so that $\mathbf{L}=\mathbf{N}_{R I}, \mathbf{R}=\mathbf{N}_{R O}$ and

$$
\mathbf{M}=\mathbf{N}_{R O} \mathbf{Z N}_{R I}, \quad \mathbf{Z}=\mathbf{N}_{R O}^{T} \mathbf{M N}_{R I}^{T}
$$

\subsection{Physical invariants of a Mueller matrix}

Having extracted the overall retarding properties exhibited by the medium represented by $\mathbf{M}$ and characterized by means of the matrices $\mathbf{N}_{R I}$ and $\mathbf{N}_{R O}$, we will analyze the properties and physical information contained in $\mathbf{Z}$.

Concerning the matrix $\mathbf{D}$, it is easy to show that this is a Stokes matrix. Nevertheless, taking into account equation (245), the following inequality is required for $\mathbf{D}$ to satisfy the eigenvalue conditions

$$
d_{0}+d_{3} \geqslant d_{1}+d_{2}
$$

whereas the condition for $\mathbf{D}$ to satisfy the transmittance conditions is

$$
d_{0} \leqslant 1
$$

Moreover, $\mathbf{U}$ and $\mathbf{V}$ are not Mueller matrices, in such a manner that the serial combination given by equation (252) is always a Mueller matrix.

By examining the nature of these different matrices, we see that $\mathbf{D}$ always exhibits depolarizing properties, including the case where $\mathbf{M}$ represents a pure medium. We observe that $P_{(4)}(\mathbf{D})<1$, except for the degenerate case $d_{0}=d_{1}=d_{2}=d_{3}$ corresponding to a retarder $\mathbf{M}=d_{0} \mathbf{N}_{R O} \mathbf{N}_{R I}$ with transmittance $d_{0}$. On the other hand, the non-Mueller matrices $\mathbf{U}$ and $\mathbf{V}$ produce "overpolarizing" effects, so that the distances to the origin of the points representing some states on the corresponding P-Images (or DoP surfaces) are higher than 1 .

As we have seen, all the depolarization properties are given by the three indices of purity $P_{i}$, which are nondimensional magnitudes defined from the eigenvalues of the coherency matrix $\mathbf{H}$ associated with $\mathbf{M}$ [41]. These quantities are invariant under changes of the laboratory reference axis, as well as changes of the generalized basis used for the representation of the states of polarization.

Usually, linear polarizations along the axes $X Y$ are used as physical reference axes, so that in the Poincaré sphere representation the Stokes parameter $s_{1}$ corresponds to the $X$ axis, $s_{2}$ corresponds to the $X Y$ bisector axis and $s_{3}$ corresponds to right-handed circularly polarized light. Nevertheless, it is possible to construct different physical bases by choosing orthogonal pairs of circularly or elliptically polarized light. All the possible changes of the reference basis for the representation of Stokes vectors correspond to rotations in the Poincaré sphere and, in consequence, are performed by means of similarity transfor- mations whose orthogonal matrix has the form of the $\mathbf{R}$, $\mathbf{L}$ matrices, and not the form of the $\mathbf{U}, \mathbf{V}$ matrices.

According to the SVD of $\mathbf{Z}, \mathbf{U}$ and $\mathbf{V}$ are the "output" and "input" orthogonal non-Mueller matrices, whose respective columns are the output and input singular vectors of $\mathbf{Z}$. Moreover, if we consider the SVD of the complete Mueller matrix $\mathbf{M}$, the orthogonal matrices $\mathbf{N}_{R I}$ and $\mathbf{N}_{R O}$ represent the input and output orthogonal physical transformations, i.e. respective rotations in the Poincaré sphere. Thus, different respective bases for the representation of input and output Stokes vectors can be specified, so that the form of $\mathbf{N}_{R I}$ and $\mathbf{N}_{R O}$ depend on the particular bases chosen, whereas the kernel matrix $\mathbf{Z}$ is physically invariant. No physical transformation can produce changes in $\mathbf{Z}$. Hence, all quantities derived from $\mathbf{Z}$ are physical invariants. The input and output equivalent retarders $\mathbf{N}_{R I}$, $\mathbf{N}_{R O}$ depend on three respective independent parameters, whereas $\mathbf{Z}$ depends on ten independent parameters.

Thus, it is easy to show that the indices of purity (and hence the degree of purity) of $\mathbf{Z}$ are the same than those of $\mathbf{M}$. It is also important to note that $\mathbf{Z}$ and $\mathbf{M}$ have the same transmittance for unpolarized light

$$
\begin{aligned}
P_{i}(\mathbf{Z}) & =P_{i}(\mathbf{M}), \quad i=1,2,3 \\
z_{00} & =m_{00}
\end{aligned}
$$

Concerning the polarizances of $\mathbf{Z}$, it is easy to show that, as expected, their values are also physical invariants,

$$
\Upsilon_{f}(\mathbf{Z})=\Upsilon_{f}(\mathbf{M}), \quad \Upsilon_{r}(\mathbf{Z})=\Upsilon_{r}(\mathbf{M})
$$

Nevertheless the polarizance vectors $\Upsilon_{r}, \Upsilon_{f}$ are not physically invariant

$$
\mathbf{\Upsilon}_{f}(\mathbf{Z}) \neq \mathbf{\Upsilon}_{f}(\mathbf{M}), \quad \mathbf{\Upsilon}_{r}(\mathbf{Z}) \neq \mathbf{\Upsilon}_{r}(\mathbf{M})
$$

and, therefore, they must be distinguished from the "intrinsic polarizance vectors" $\boldsymbol{\Gamma}_{f}, \boldsymbol{\Gamma}_{r}$ defined as

$$
\boldsymbol{\Gamma}_{f} \equiv \frac{1}{m_{00}}\left(z_{10}, z_{20}, z_{30}\right)^{T}, \quad \boldsymbol{\Gamma}_{r} \equiv \frac{1}{m_{00}}\left(z_{01}, z_{02}, z_{03}\right)^{T},
$$

which depend only on the elements of $\mathbf{Z}$ and, hence, are physically invariant.

In consequence, a complete description of the objective physical quantities embedded in $\mathbf{M}$ is the following:

(a) 6 parameters of overall birefringence:

- "input birefringence", given by $\mathbf{N}_{R I}\left(\alpha_{i}, \delta_{i}, \Delta_{i}\right)$,

- "output birefringence", given by $\mathbf{N}_{R O}\left(\alpha_{o}, \delta_{o}, \Delta_{o}\right)$;

(b) 10 physically invariant parameters:

- transmittance for unpolarized light ("mean transmittance") $m_{00}$,

- indices of purity $P_{1}, P_{2}, P_{3}$, which characterize completely the polarimetric purity of the system,

- intrinsic polarizance vectors $\boldsymbol{\Gamma}_{f}, \boldsymbol{\Gamma}_{r}$ (we observe that the polarizances $\Upsilon_{f}(\mathbf{M}), \Upsilon_{r}(\mathbf{M})$ are the respective modulus of these vectors). 
The obtainment of this set of objective physical parameters constitutes an important mean of exploiting the polarimetric techniques, as well as a tool for analyzing experimental results. In particular, not only the mathematical invariants $\left(m_{00}, P_{1}, P_{2}, P_{3}\right)$, but all the physical invariants $\left(m_{00}, P_{1}, P_{2}, P_{3}, \boldsymbol{\Gamma}_{f}^{T}, \boldsymbol{\Gamma}_{r}^{T}\right)$ can be used for the objective characterization of material samples.

We observe that the indices of purity can be obtained directly from numeric calculus of the eigenvalues of the coherency matrix $\mathbf{H}$ associated with the measured Mueller matrix $\mathbf{M}$. To complete the calculation of the set of objective physical quantities, we present below a procedure to obtain the parameters of the SVD of $\mathbf{M}$

- through numeric calculus, the singular values $d_{0}, d_{1}, d_{2}, d_{3}$ are calculated as the positive square roots of the eigenvalues of the positive semidefinite symmetric matrix $\mathbf{M}^{T} \mathbf{M}=\mathbf{N}_{R I}^{T} \mathbf{V}^{T} \mathbf{D}^{2} \mathbf{V} \mathbf{N}_{R I}$;

- the eigenvectors of $\mathbf{M}^{T} \mathbf{M}$, which are the rows of the diagonalization orthogonal matrix $\mathbf{V} \mathbf{N}_{R I}$ are then calculated. From now on, the elements of the matrix $\mathbf{V N}_{R I}$ can be used as data;

- the elements of $\mathbf{V}$ are calculated from the first column of $\mathbf{V N}_{R I}$ and, hence, all the angles characterizing $\mathbf{V}$ and $\mathbf{N}_{R I}$ are calculated;

- in the same way, the eigenvectors of $\mathbf{M M}^{T}=$ $\mathbf{N}_{R O} \mathbf{U D}^{2} \mathbf{U}^{T} \mathbf{N}_{R O}^{T}$ are numerically calculated so that, once is $\mathbf{N}_{R O} \mathbf{U}$ obtained, its elements can be used as data;

- the elements of $\mathbf{U}$ are calculated from the first row of $\mathbf{N}_{R O} \mathbf{U}$ and, hence, all the angles characterizing $\mathbf{U}$ and $\mathbf{N}_{R O}$ are obtained.

Some important theorems derived from the SVD of $\mathbf{M}$ are the following

(a) the diagonal matrix $\mathbf{D}$, corresponding to the SVD of $\mathbf{M}$, is a Stokes matrix;

(b) if the kernel matrix $\mathbf{Z}$ is diagonal, then $\Upsilon_{f}=\Upsilon_{r}=0$;

(c) any Mueller matrix $\mathbf{M}$ that satisfies $\Upsilon_{f}=\Upsilon_{r}=0$ can be written as $\mathbf{M}=\mathbf{N}_{R O} \mathbf{D N}_{R I}$. This matrix corresponds to a parallel combination of retarders and depends on up to ten independent parameters;

(d) $\Upsilon_{f}=\Upsilon_{r}=1 \Rightarrow P_{(4)}=1$;

(e) an ideal depolarizer can be synthesized through the parallel combination of pure retarders given by equation (202);

(f) $P_{(4)}^{2}(\mathbf{Z})=P_{(4)}^{2}(\mathbf{M})=\frac{1}{3}\left(\frac{\sum_{i=0}^{3} d_{i}^{2}}{m_{00}^{2}}-1\right)$ $=P_{(4)}^{2}(\mathbf{D})+\frac{d_{0}^{2}-m_{00}^{2}}{3 m_{00}^{2}} ;$

(g) $d_{0}^{2} \geqslant m_{00}^{2} ; \quad d_{0}^{2}=m_{00}^{2} \Leftrightarrow \mathbf{Z}=\mathbf{D}$;

(h) $P_{(4)}^{2}(\mathbf{Z})=P_{(4)}^{2}(\mathbf{M})=\frac{1}{3}\left(\Upsilon_{f}^{2}+\Upsilon_{r}^{2}+\frac{\sum_{i, j=1}^{3} z_{i j}^{2}}{m_{00}^{2}}\right)$;

(i) $\mathbf{Z}$ is a Mueller-Jones matrix if, and only if, the following equalities are satisfied $\mathbf{Z}=\mathbf{Z}^{T}\left(\varphi_{1}=\varphi_{4}, \varphi_{2}=\varphi_{5}\right.$, $\left.\varphi_{3}=\varphi_{6}\right), \varphi_{1}=\pi / 4, d_{2}=d_{3}$.
An examination of the kernel matrix enables the following classification of the measured media to be made:

- $\mathbf{Z} \neq \mathbf{Z}^{T}$ : the system represented by $\mathbf{M}$ is optically equivalent to a parallel combination of pure diattenuators and pure retarders placed between two pure retarders. It would be interesting to analyze the nondemonstrated hypothesis that, in this case, $\mathbf{Z}$ is a parallel combination of non-homogeneous diattenuators;

- $\mathbf{Z}=\mathbf{Z}^{T}, \mathbf{Z} \neq \mathbf{D}$ : the system represented by $\mathbf{M}$ is optically equivalent to a parallel combination of pure diattenuators placed between two pure retarders;

- $\mathbf{Z}=\mathbf{D}$ : the system represented by $\mathbf{M}$ is optically equivalent to a parallel combination of pure retarders. $\mathbf{Z}$ represents a depolarizer (also called a "pure depolarizer" [149] but, in order to avoid confusions, we retain the term "pure" to refer to deterministic nondepolarizing systems).

\section{Some applications of polarimetry}

The term "polarimetry" extends over a wide range of scientific, medical and industrial applications. An exhaustive summary of all these applications would require a voluminous treatise. Nevertheless, in order to provide a brief panoramic view, we include here some references to several fields where the polarimetric techniques play an important role.

Light scattering [8,34,215,216]: remote sensing [217], lidar, atmospheric phenomena, aerosols, hydrosols, surface roughness [26,218-222], particle sizing, particle characterization (contaminants, biological microorganisms... ), surface characterization...

Optical fiber and photonic devices [145,146,223,228]: communication systems; characterization and control of polarization mode dispersion, fiber optic sensors...

Synthetic aperture radar $(S A R)$ polarimetry $[59,195$, 229]: airborne and spaceborne remote sensing, imaging and detection; vegetation, agriculture, crop classification, forestry, sea ice, sandy areas; Geoscience [230], digital terrain models, ground topographic mapping, Meteorology, observation and prediction of hurricanes, detection of devastated areas, oil spills, immersed targets, buried targets, anti-personnel mines [231]...

Medicine \& Biology [232-236]: study, detection and imaging of immunological reactions, biological tissues, optical coherence tomography [232,233, 237-240], human eye [241,242], oral precancer [209], DNA structure... 244].

Experimentation under microgravity conditions [243,

Photoelasticity [127, 245,246].

Astronomy \& Astrophysics [36,247]: X-ray polarimetry, Solar polarimetry, interstellar dust, planetary atmospheres, black holes, satellite missions...

Optics industry \& research: fabrication [248], characterization of optical components [249], analysis of optical systems [250,251], ray tracing [252], spectral filters design and fabrication [253]...

Imaging polarimetry [32,233, 239, 241,254-257]. 
Plasma physics, nuclear fusion, ITER, Tokamak [35, 38]; synchrotron, particle physics...

Microelectronics Industry \& Metrology [258,259].

Research in Quantum Physics [85,96,260-262]: Bell inequalities, optical computing, teleportation, cryptology. . .

LCD Technologies, [263-265].

Thin films, Stratified \& Layered Media [266-268].

Microwave transmission systems [269].

The author would like to thank Drs. I. San José, J.M. Correas, C. Ferreira, P. Melero and J. Delso, colleagues of the Group of Optical Polarimetry, for their helpful comments. The author is also obliged to Dr. R. Navarro for his valuable advice and encouragement in writing this paper.

\section{References}

1. R. Loudon, The quantum theory of light (Clarendon, Oxford, 1983)

2. C. Brosseau, Fundamentals of polarized light. A statistical approach (Wiley, N.Y., 1998)

3. G.G. Stokes, Trans. Cambridge Phil. Soc. 9, 399 (1852)

4. U. Fano, Phys. Rev. 93, 121 (1953)

5. R. Barakat, Opt. Acta 32, 295 (1985)

6. E. Wolf, Nuovo Cim. 13, 1165 (1959)

7. L. Mandel, Proc. Phys. Soc. 81, 1104 (1963)

8. C.F. Bohren, D.R. Huffman, Absorption and Scattering of Light by Small Particles (Wiley-Interscience, N.Y., 1983)

9. R.C. Jones, J. Opt. Soc Am. 31, 488 (1941)

10. P. Soleillet, Ann. Phys. Fr. 12, 23 (1929)

11. F. Perrin, J. Chem. Phys. 10, 415 (1942)

12. H. Mueller, J. Opt. Soc. Am. 38, 661 (1948)

13. N.G. Parke III, Matrix optics, Ph.D. thesis, M.I.T., 1948

14. N.G. Parke III, J. Math. Phys. 28, 131 (1949)

15. Benchmark Papers in Optics v1: Polarized Light, edited by W. Swindell (Dowden, Hutchinson and Ross, Stroudsburg, 1975)

16. J.J. Gil, E. Bernabéu, Optik 76, 67 (1987)

17. S.-Y. Lu, R.A. Chipman, J. Opt. Soc. Am. A 11, 766 (1994)

18. R.M.A. Azzam, N.M. Bashara, Ellipsometry and Polarized Light (North-Holland, N.Y., 1977)

19. M. Schubert, Ann. Phys. 15, 480 (2006)

20. R.M.A. Azzam, Opt. Lett. 2, 148 (1978)

21. P.S. Hauge, J. Opt. Soc. Am. 68, 1519 (1978)

22. R.C. Thompson, J.R. Bottiger, E.S. Fry, Appl. Opt. 19, 1323 (1980)

23. E. Bernabéu, J.J. Gil, J. Opt. 16, 139 (1985)

24. D.B. Chenault, J.L. Pezzanity, R.A. Chipman, SPIE 1746, 231 (1992)

25. Polarization Analysis and Measurement, edited by D.H. Goldstein, R.A. Chipman, Proc. SPIE 1746 (1992)

26. F. Delplancke, Appl. Opt. 36, 5388 (1997)

27. M. Mujat, A. Dogariu, Appl. Opt. 40, 34 (2001)

28. K. Ichimoto, K. Shinoda, T. Yamamoto, J. Kiyohara, Publ. Natl. Astron. Obs. Jap. 9, 11 (2006)

29. R.M.A. Azzam, Optik 52, 253 (1979)

30. F. Gori, Opt. Lett. 24, 584 (1999)

31. J.-K. Lee, J.T. Shen, A. Heifetz, R. Tripathi, M.S. Shahriar, Opt. Commun. 259, 484 (2006)
32. S. Tyo, D.L. Goldstein, D. Chenault, J.A. Shaw, Appl. Opt. 45, 5453 (2006)

33. H.C. van de Hulst, Light scattering by small particles (Wiley, N.Y., 1957), Chap. 5

34. Light scattering by Microstructures, edited by F. Moreno, F. González (Lecture Notes in Physics, Springer-Verlag, Berlin, 2000)

35. T. Kondoh, A.E. Costley, T. Sugie, Y. Kawano, A. Malaquias, C.I. Walker, Rev. Sci. Instrum. 75, 3420 (2004)

36. C. Heiles, P. Perillat, M. Nolan, D. Lorimer, R. Bhat, T. Ghosh, M. Lewis, K. O'Neil, C. Salter, S. Stanimirovic, Publ. Astron. Soc. Pacific 113, 1274 (2001); available from <http://www. journals.uchicago.edu/ cgi-bin/ resolve?PASP201143PDF>

37. A.L. Fymat, Opt. Eng. 20, 25 (1981)

38. J.H. Rommers, S. Barry, R. Behn, C. Nieswand, Plasma Phys. Control. Fusion 40, 2073 (1998)

39. M. Mujat, E. Baleine, A. Dogariu, J. Opt. Soc. Am. A 21, 2244 (2004)

40. D.T. Chuss, E.J. Wollack, S.H. Moseley, G. Novak, Appl. Opt. 45, 5107 (2006)

41. J.J. Gil, J.M. Correas, P.A. Melero, C. Ferreira, Monogr. Sem. Mat. García de Galdeano 31, 161 (2004); available from <http://www . unizar.es/galdeano/actas_pau/ PDFVIII/pp161-167.pdf $>$

42. T. Tudor, I. Vinkler, Pure Appl. Opt. 7, 1451 (1998)

43. N. Wiener, Acta Math. 55, 182 (1930)

44. G.B. Parrent, P. Roman, Nuovo Cim. 15, 370 (1960)

45. R. Barakat, J. Opt. Soc. Am. 53, 317 (1963)

46. U. Fano, Rev. Mod. Phys. 29, 74 (1957)

47. W.H. McMaster, J. Opt. Soc. Am. 22, 351 (1954)

48. S.R. Cloude, in Direct and Inverse Methods in Radar Polarimetry, Part I, edited by W.M. Boerner et al. (Kluwer Academic Publishers, Dordrecht, The Netherlands, 1992)

49. E.L. O'Neill, Introduction to Statistical Optics (AddisonWesley, Reading, Mass., 1963), Chap. 9

50. A. Al-Qasimi, O. Korotkova, D. James, E. Wolf, Opt. Lett. 32, 1015 (2007)

51. J. Ellis, A. Dogariu, J. Opt. Soc. Am. A 21, 988 (2004)

52. A. Luis, Phys. Rev. A 75, 053806 (2007)

53. R. Barakat, Opt. Acta 30, 1171 (1983)

54. R. Barakat, C. Brosseau, J. Opt. Soc. Am. A 10, 529 (1992)

55. R. Barakat, Opt. Commun. 123, 443 (1996)

56. C. Brosseau, D. Bicout, Phys. Rev. E 50, 4997 (1994)

57. S.R. Cloude, E. Pottier, Opt. Eng. 34, 1599 (1995)

58. F. Le Roy-Brehonnet, B. Le Jeune, P.Y. Gerligand, J. Cariou, J. Lotrian, Pure Appl. Opt. 6, 385 (1997)

59. W.-M. Boerner, in Radar Polarimetry and Interferometry (Educational Notes RTO-EN-SET-081bis, Paper 12. Neuilly-sur-Seine, France: RTO p. 12-1, 2007); available form <http://www.rto.nato.int/abstracts.asp $>$

60. A.F. Fercher, P.F. Steeger, Opt. Acta 28, 443 (1981)

61. R. Barakat, J. Opt. Soc. Am. A 4, 1256 (1987)

62. C. Brosseau, J. Opt. Soc. Am. A 6, 649 (1989)

63. C. Brosseau, R. Barakat, E. Rockower, Opt. Commun. 82, 204 (1991)

64. C. Brosseau, J. Mod. Opt. 39, 1167 (1992)

65. C. Brosseau, Appl. Opt. 34, 4788 (1995)

66. S. Pancharatnam, Proc. Ind. Acad. Sci. A 44, 247 (1956)

67. S. Pancharatnam, Proc. Ind. Acad. Sci. A 44, 398 (1956) 
68. F. Gori, Opt. Lett. 23, 241 (1998)

69. J.M. Movilla, G. Piquero, R. Martínez-Herrero, P.M. Mejías, Opt. Commun. 149, 230 (1998)

70. J. Tervo, T. Setälä, A.T. Friberg, Opt. Express 11, 1137 (2003)

71. J. Ellis, A. Dogariu, Opt. Lett. 29, 536 (2004)

72. J. Tervo, T. Setälä, A.T. Friberg, J. Opt. Soc. Am. A 21, 2205 (2004)

73. P. Réfrégier, F. Goudail, Opt. Express 13, 6051 (2005)

74. R. Castañeda, Opt. Commun. 267, 4 (2006)

75. O. Korotkova, E. Wolf, Opt. Lett. 30, 1 (2005)

76. E. Wolf, Phys. Lett. A 312, 263 (2003)

77. A. Luis, Opt. Commun. 251, 243 (2005)

78. A. Luis, Opt. Commun. 263, 141 (2006)

79. A. Luis, Opt. Commun. 246, 437 (2005)

80. O. Korotkova, E. Wolf, J. Mod. Opt. 52, 2659 (2005)

81. O. Korotkova, E. Wolf, J. Mod. Opt. 52, 2673 (2005)

82. E. Collett, Am. J. Phys. 38, 563 (1969)

83. A. Luis, Phys. Rev. A 66, 013806 (2002)

84. A. Luis, Phys. Rev. A 71, 063815 (2005)

85. A. Luis, N. Korolkova, Phys. Rev. A 74, 043817 (2006)

86. A. Luis, Opt. Commun. 273, 173 (2007)

87. P. Roman, Nuovo Cim. 13, 2546 (1959)

88. A. Papoulis, Probability, random variables and stochastic processes (Mac Graw-Hill, Singapur, 1984)

89. M.R. Dennis, J. Opt. A: Pure Appl. Opt. 6, S26 (2004)

90. J.H. Hannay, J. Mod Opt. 45, 1001 (1998)

91. T. Saastamoinnen, J. Tervo, J. Mod. Opt. 51, 2039 (2004)

92. J. Ellis, A. Dogariu, Opt. Commun. 253, 257 (2005)

93. T. Carozzi, R. Karlsson, J. Bergman, Phys. Rev. E 61, 2024 (2000)

94. T. Setälä, A. Shevchenko, M. Kaivola, A.T. Friberg, Phys. Rev. E 66, 016615 (2002)

95. A. Luis, Opt. Commun. 253, 10 (2005)

96. A.P. Alodzhants, S.M. Arakelian, Opt. Spectrosc. 97, 453 (2004)

97. A.P. Alodzhants, S.M. Arakelian, J. Mod. Opt. 46, 475 (1999)

98. T. Setälä, M. Kaivola, A.T. Friberg, Phys. Rev. Lett. 88, $1239021(2002)$

99. R. Dändliker, P. Tortora, L. Vaccaro, A. Nesci, J. Opt. A: Pure Appl. Opt. 6, S18 (2004)

100. J. Ellis, A. Dogariu, Phys. Rev. Lett. 95, 203905 (2005)

101. J. Ellis, A. Dogariu, S. Ponomarenko, E. Wolf, Opt. Lett. 29, 1536 (2004)

102. J.M. Correas, P.A. Melero, J.J. Gil, Monogr. Sem. Mat. García de Galdeano 27, 23 (2003); available from $<$ http://www.unizar.es/galdeano/actas_pau/PDF/ 233.pdf $>$

103. R.A. Horn, C.R. Johnson, Matrix Analysis (Cambridge Univ. Press, 1985)

104. J.C. Samson, Geophys. J.R. Astron. Soc. 34, 403 (1973)

105. J. Ellis, A. Dogariu, S. Ponomarenko, E. Wolf, Opt. Commun. 248, 333 (2005)

106. F.T. Hioe, J. Mod. Opt. 53, 1715 (2006)

107. P. Réfrégier, M. Roche, F. Goudail, J. Opt. Soc. Am. A 23, 124 (2006)

108. P. Réfrégier, F. Goudail, J. Opt. Soc. Am. A 23, 671 (2006)

109. M.R. Dennis, J. Opt. Soc. Am. A 24, 2065 (2007)

110. A. Luis, Phys. Rev. A. 71, 1023810 (2005)

111. P. Vahimaa, J. Tervo, J. Opt. A: Pure Appl. Opt. 6, S41 (2004)
112. W.A. Holm, R.M. Barnes, in Proceedings of the 1988 IEEE USA radar conference, p. 249

113. S.R. Cloude, E. Pottier, IEEE Trans. GRS 34, 498 (1996)

114. P. Réfrégier, F. Goudail, P. Chavel, A. Friberg, J. Opt. Soc. Am. A 21, 21124 (2004)

115. P. Réfrégier, J. Morio, J. Opt. Soc. Am. A 23, 3036 (2006)

116. R. Simon, Opt. Commun. 77, 349 (1990)

117. A.P. Loeber, J. Opt. Soc. Am. 72, 650 (1982)

118. L.N. Dlugnikov, Opt. Acta 31, 803 (1984)

119. B. Chakraborty, J. Opt. Soc. Am. A 3, 1422 (1986)

120. G. Indebetouw, Optik 85, 78 (1990)

121. R. Simon, Opt. Commun. 42, 293 (1982)

122. J.W. Hovenier, Appl. Opt. 33, 8318 (1994)

123. R.C. Jones, J. Opt. Soc. Am. 32, 486 (1942)

124. R. Barakat, J. Mod. Opt. 34, 1535 (1987)

125. C. Brosseau, R. Barakat, Opt. Commun. 84, 127 (1991)

126. A.B. Kostinski, R.C. Givens, J. Mod. Opt. 39, 1947 (1992)

127. P.S. Theocaris, E.E. Gdoutos, Matrix theory of photoelasticity (Springer-Verlag, Berlin, 1979), Chap. 4

128. N. Vansteenkiste, P. Nignolo, A. Aspect, J. Opt. Soc. Am. A 10, 2240 (1993)

129. R.J. Potton, Rep. Prog. Phys. 67, 717 (2004)

130. J.J. Gil, Ph.D. thesis, Facultad de Ciencias, Univ. Zaragoza, Spain, 1983; available from <http://www.pepegil.es/PhD-Thesis-JJ-Gil.pdf>

131. J.J. Gil, E. Bernabéu, Opt. Acta 33, 185 (1986)

132. H. Hurwitz, R.C. Jones, J. Opt. Soc. Am. 31, 493 (1941)

133. J.R. Priebe, J. Opt. Soc. Am. 59, 176 (1969)

134. P.H. Richter, J. Opt. Soc. Am. 69, 460 (1979)

135. C. Whitney, J. Opt. Soc. Am. 61, 1207 (1971)

136. R. Barakat, Eur. J. Phys. 19, 209 (1998)

137. S.T. Tang, H.S. Kwok, J. Opt. Soc. Am. A 18, 2138 (2001)

138. J.J. Gil. E. Bernabéu, Opt. Pure Appl. 15, 39 (1982)

139. R.C. Jones, J. Opt. Soc. Am. 38, 671 (1948)

140. N. Go, J. Phys. Soc. Jpn 23, 88 (1967)

141. R. Barakat, J. Opt. Soc. Am. A 13, 158 (1996)

142. R.M.A. Azzam, J. Opt. Soc. Am. 68, 1756 (1978)

143. C. Brosseau, Opt. Lett. 20, 1221 (1995)

144. J.F. Mosiño, O. Barbosa-García, M.A. Meneses-Nava, L.A. Díaz-Torres, E. de la Rosa-Cruz, J.T. Vega-Durán, J. Opt. A: Pure Appl. Opt. 4, 419 (2002)

145. C.D. Poole, R.E. Wagner, Electron. Lett. 22, 1029 (1986)

146. W. Shieh, IEEE Phot. Tech. Lett. 11, 677 (1999)

147. E. Collett, Am. J. Phys. 39, 517 (1971)

148. A.S. Marathay, J. Opt. Soc. Am. 55, 969 (1965)

149. S.-Y. Lu, R.A. Chipman, J. Opt. Soc. Am. A 13, 1106 (1996)

150. C.R. Fernández-Pousa, I. Moreno, N. Bennis, C. GómezReino, C. Ferreira, Opt. Commun. 183, 347 (2000)

151. R.M.A. Azzam, J. Opt. Soc. Am. 62, 1252 (1972)

152. Z.-.F Xing, J. Mod. Opt. 39, 461 (1992)

153. S.N. Savenkov, O. I Sydoruk, R.S. Muttiah, J. Opt. Soc. Am. A 22, 1447 (2005)

154. G.S. Agarwal, Opt. Commun. 82, 213 (1991)

155. S.N. Savenkov, V.V. Marienko, E.A. Oberemok, Phys. Rev. E 74, 056607 (2006)

156. M.V. Berry, M.R. Dennis, J. Opt. A: Pure Appl. Opt. 6, S24 (2004)

157. H. Takenaka, Nouv. Rev. Opt. 4, 37 (1973)

158. Sudha, A.V. Gopala, J. Opt. Soc. Am. A 18, 3130 (2001)

159. C.S. Brown, A.E. Bak, Opt. Eng. 34, 1625 (1995) 
160. R. Sridhar, R. Simon, J. Mod. Opt. 41, 1903 (1994)

161. S.R. Cloude, Optik 75, 26 (1986)

162. S.R. Cloude, J. Electromag. Waves Appl. 6, 947 (1992)

163. S.R. Cloude, SPIE 2256, 292 (1994)

164. E.S. Fry, G.W. Kattawar, Appl. Opt. 20, 3428 (1981)

165. K. Kim. L. Mandel, E. Wolf, J. Opt. Soc. Am. A 4, 433 (1987)

166. S.R. Cloude, SPIE 1166, 177 (1989)

167. C.V.M. van der Mee, J. Math. Phys. 34, 5072 (1993)

168. J.J. Gil, J. Opt. Soc. Am. A 17, 328 (2000)

169. C.V.M. van der Mee, J.W. Hovenier, J. Math. Phys. 33, 3574 (1992)

170. D.I. Nagirner, Astron. Astrophys. 275, 318 (1993)

171. M.S. Kumar, R. Simon, Opt. Commun. 11, 2305 (1994)

172. C.R. Givens, A.B. Kostinski, J. Mod. Opt. 40, 471 (1993)

173. A.B. Kostinski, C.R. Givens, J.M. Kwiatkowski, Appl. Opt. 32, 1646 (1993)

174. A.V. Gopala, K.S. Mallesh, Shuda, J. Mod. Opt. 45, 955 (1998)

175. A.V. Gopala, K.S. Mallesh, Shuda, J. Mod. Opt. 45, 989 (1998)

176. J.J. Gil, E. Bernabeu, Opt. Acta 32, 259 (1985)

177. J.W. Hovenier, H.C. van de Hulst, C.V.M. van der Mee, Astron. Astrophys. 157, 301 (1986)

178. R. Simon, J. Mod. Opt. 34, 569 (1987)

179. G. Arfken, Mathematical methods for physicists (Academic Press, 1970), Chap. 4

180. A. Aiello, G. Puentes, J.P. Woerdman, arXiv: quant-ph/0611179v1 (2006)

181. Sudha, A.V. Gopala Rao, A.R. Usha Devi, A.K. Rajagopal, arXiv:0704.0147v2 [physics.optics] (2007)

182. D.G.M. Anderson, R. Barakat, J. Opt. Soc. Am. A 11, 2305 (1994)

183. I. Kuscer, M. Ribaric, Opt. Acta 6, 42 (1959)

184. K.D. Abhyankar, A.L. Fymat, J. Math. Phys. 10, 1935 (1969)

185. R. Barakat, Opt. Commun. 38, 159 (1981)

186. C. Brosseau, C.R. Givens, A.B. Kostinski, J. Opt. Soc. Am. A 10, 2248 (1993)

187. J.J. Gil, in Light scattering from microestructures (Springer, Lecture Notes in Physics, 2000)

188. R. Espinosa-Luna, Appl. Opt. 46, 6047 (2007)

189. C. Brosseau, Optik 85, 83 (1990)

190. A.B. Kostinski, Appl. Opt. 31, 3506 (1992)

191. F. Le Roy-Brehonnet, B. Le Jeune, Prog. Quant. Electr. 21, 109 (1997)

192. F. Le Roy-Brehonnet, B. Le Jeune, P. Eliès, J. Cariou, J. Lotrian, J. Phys. D: Appl. Phys. 29, 34 (1996)

193. A. Aiello, J.P. Woerdman, Phys. Rev Lett. 94, 090406 (2005)

194. R.A. Chipman, Appl. Opt. 44, 2490 (2005)

195. R. Touzi, W.M. Boerner, J.S. Lee, E. Lueneburg, Can. J. Remote Sensing 30, 380 (2004); available from $<$ www.radarsat2.info/outreach/resources/Reviewof-Polarimetry.pdf $>$

196. C. Brosseau, Opt. Commun. 131, 229 (1996)

197. F. Le Roy-Bréhonnet, B. Le Jeune, P.Y. Gerligand, J. Cariou, J. Lotrian, Pure Appl. Opt. 6, 385 (1996)

198. B. DeBoo, J. Sasian, R. Chipman, Opt. Express 12, 4941 (2004)

199. C. Ferreira, I. San José, J.J. Gil, J.M. Correas, Monogr. Sem. Mat. García de Galdeano 33, 115 (2006); available from <http://www. unizar.es/galdeano/actas_pau/ PDFIX/FerSanGilCor05.pdf $>$
200. R.A. Chipman, in The Handbook of Optics (McGraw-Hill, N.Y., 1994), Chap. 22

201. G. Puentes, D. Voigt, A. Aiello, J.P. Woerdman, Opt. Lett. 30, 3216 (2005)

202. J.J. Gil, SPIE 5622, 725 (2004)

203. E.C.G. Sudarshan, Phys. Rev. 121, 920 (1961)

204. G. Kimura, Phys. Lett. A 314, 339 (2003)

205. G.L. Long, Y.-F. Zhou, J.-Q. Jin, Y. Sun, H.-W. Lee, Found. Phys. 36, 1217 (2006)

206. S. Lu, A.P. Loeber, J. Opt. Soc. Am. 65, 248 (1975)

207. W.A. Shurcliff, Polarized light (Harvard U. Press, Cambridge, Mass., 1962)

208. S.-Y. Lu, R.A. Chipman, Opt. Commun. 146, 11 (1998)

209. J. Chung, W. Jung, M.J. Hammer-Wilson, P. WilderSmith, Z. Chen, Appl. Opt. 46, 3038 (2007)

210. M.K. Swami, S. Manhas, P. Buddhiwant, N. Ghosh, A. Uppal, P.K. Gupta, Opt. Express 14, 9324 (2006)

211. C. Collet, J. Zallat, Y. Takakura, Opt. Express 12, 1271 (2004)

212. J. Morio, F. Goudail, Opt. Lett. 29, 2234 (2004)

213. R. Ossikovski, A. De Martino, S. Guyot, Opt. Lett. 32, 689 (2007)

214. M. Anastasiadou, S. Ben Hatit, R. Ossikovski, S. Guyot, A. De Martino, J. Eur. Opt. Soc. Rapid Pub. 2, 07018 (2007)

215. M.I. Mishchenko, L.D. Travis, A.A. Lacis, Scattering, Absorption and Emission of Light by Small Particles (Cambridge University Press, Cambridge, UK, 2002)

216. M.I. Mishchenko, J.W. Hovenier, L.D. Travis, Light scattering by nonspherical particles (Academic Press, San Diego, 1999)

217. Photopolarimetry in Remote Sensing, edited by G. Videen, Y. Yatskiv, M.I. Mishchenko, Proceedings of the NATO Advanced Study Institute, Yalta, 2003

218. B.J. DeBoo, J.M. Sasian, R.A. Chipman, Appl. Opt. 44, $5434(2005)$

219. R. Espinosa-Luna, G. Atondo-Rubio, A. MendozaSuárez, Opt. Commun. 257, 62 (2006)

220. G. Videen, J.-Y. Hsu, W.S. Bickel, W.L. Wolfe, J. Opt. Soc. Am. A 9, 1111 (1992)

221. R. Espinosa-Luna, A. Mendoza-Suárez, G. AtondoRubio, S. Hinojosa, J. Rivera-Vázquez, J.T. GuillénBonilla, Opt. Commun. 259, 60 (2006)

222. S. Hinojosa, R. Espinosa-Luna, Opt. Commun. 250, 213 (2005)

223. E. Collett, Polarized light in Fiber Optics (PolaWave Group, Lincroft, N.J., 2003)

224. J.P. Gordon, H. Kogelnik, Proc. Natl Acad. Sci. USA 97, 4541 (2000)

225. H. Dong, P. Shum, M. Yan, J.Q. Zhou, G.X. Ning, Opt. Express 14, 5067 (2006)

226. P. Olivard, P.Y. Gerligand, B. Le Jeune, J. Cariou, J. Lotrian, J. Phys. D: Appl. Phys. 32, 1618 (1999)

227. S.X. Wang, A.M. Weiner, Opt. Lett. 29, 923 (2004)

228. F. Tang, X.-Z. Wang, Y. Zhang, W. Jing, Appl. Opt. 46, 4073 (2007)

229. ESA Proceedings of POLinSAR2003: Workshop on Applications of SAR Polarimetry and Polarimetric Interferometry; available from $<$ http: //earth.esa.int/ workshops/polinsar2003/pr.html>

230. Radar Polarimetry for Geoscience Applications, edited by F.T. Ulaby (Artech House Remote Sensing Library, 1990) 
231. L. Sagués, J.M. López-Sánchez, J. Fortuny, X. Fàbregas, A. Broquetas, A.J. Sieber, in Ultra-Wideband, ShortPulse Electromagnetics 5, edited by P.D. Smith, S.R. Cloude (Springer, US, 2002)

232. S.L. Jacques, R.J. Roman, K. Lee, J. Biomed. Opt. 7 , $329(2002)$

233. J.S. Baba, J.-R. Chung, A.H. DeLaughter, B.D. Cameron, G.L. Coté, J. Biomed. Opt. 7, 341 (2002)

234. A. Lompado, M.H. Smith, V. Krishnaswamy, Proc. SPIE 4263, 161 (2001)

235. S. Guyot, M. Anastasiadou, E. Deléchelle, A. De Martino, Opt. Express 15, 7393 (2007)

236. F. Boulvert, Y. Piederrière, G. Le Brun, B. Le Jeune, J. Cariou, Opt. Commun. 272, 534 (2007)

237. Coherence Domain Optical Methods and Optical Coherence Tomography in Biomedicine VIII, edited by V.V. Tuchin, J.A. Izatt, J.G. Fujimoto, Proc. SPIE 5316, 2004

238. M. Todorovic, S. Jiao, L.V. Wang, G. Stoica, Opt. Lett. 29, $2402(2004)$

239. S. Jiao, L.V. Wang, J. Biomed. Opt. 7, 350 (2002)

240. S. Jiao, L.V. Wang, Opt. Lett. 27, 101 (2002)

241. J.M. Bueno, F. Vargas-Martín, Appl. Opt. 41, 116 (2002)

242. J.M. Bueno, J. Opt. A: Pure Appl. Opt. 6, S91 (2004)

243. S.N. Savenkov, L.T. Mishchenko, R.S. Muttia, Y.A. Oberemok, I.A. Mishchenko, J. Quant. Spectrosc. Radiat. Transfer 88, 327 (2004)

244. A.C. Levasseur-Regourd, in Photopolarimetry in Remote Sensing (NATO Science Series II: Mathematics, Physics and Chemistry, Vol. 161, 2005)

245. H. Yang, S. Gibson, R. Tomlinson, Exp. Mech. 46, 619 (2006)

246. G.C. Gaunaurd, Opt. Eng. 42, 467 (2003)

247. S. Larsson, M. Pearce, Nucl. Instr. and Meth. A 525, 148 (2004)

248. J. Wolfe, R.A. Chipman, Opt. Express 12, 3443 (2004)
249. P.-Y. Gerligand, M. Smith, R.A. Chipman, Opt. Express 4, $420(1999)$

250. R.A. Chipman, Opt. Eng. 28, 90 (1989)

251. R.A. Chipman, Proc. SPIE 1166, 79 (1989)

252. R.A. Chipman, Opt. Eng. 34, 1636 (1995)

253. I. Abdulhalim, Opt. Commun. 267, 36 (2006)

254. J. L Pezzaniti, R.C. Chipman, Opt. Eng. 34, 1558 (1995)

255. P. Réfrégier, F. Goudail, N. Roux, J. Opt. Soc. Am. A 21, 2292 (2004)

256. D. Lara, C. Dainty, Appl. Opt. 45, 1917 (2006)

257. C. Kieleck, M. Floc'h, G. Le Brun, J. Cariou, J. Lotrian, Pure Appl. Opt. 6, 749 (1997)

258. T. Novikova, A. De Martino, S.B. Hatit, B. Drévillon, Appl. Opt. 45, 3688 (2006)

259. T. Novikova, A. De Martino, P. Bulkin, Q. Nguyen, B. Drévillon, Opt. Express 15, 2033 (2007)

260. A. Ekert, A. Zeilinger, The Physics of Quantum Information, edited by D. Bouwmeester (Springer, Berlin, 2000)

261. G.E. Stedman, J. Mod. Opt. 36, 461 (1989)

262. M. Takeuchi, T. Takano, S. Ichihara, A. Yamaguchi, M. Kumakura , T. Yabuzaki, Y.Takahashi, Appl. Phys. B 83, 33 (2006)

263. C. Bohley, T, Scharf, J. Opt. A: Pure Appl. Opt. 6, S77 (2004)

264. I. Dahl, Meas. Sci. Technol. 12, 1938 (2001)

265. J.L. Pezzaniti, S.C. McClain, R.A. Chipman, S.-Y. Lu, Opt. Lett. 18, 2071 (1993)

266. K.L. Woon, M. O'Neill, G.J. Richards, M.P. Aldred, S.M. Kelly, J. Opt. Soc. Am. A 22, 760 (2005)

267. P. Yeh, Optical waves in layered media (Wiley Series in Pure and Applied Optics, 1988)

268. C. Chou, H.-K. Teng, C.-J. Yu, H.-S. Huang, Opt. Commun. 273, 74 (2007)

269. H. Liu, X. Zhang, K. Chen, Opt. Commun. 236, 109 (2004) 